Florida International University FIU Digital Commons

$10-26-2016$

\title{
A Morphological, Functional, and Genetic Investigation of the Male Compound Eye Phenotype of Chrysomya megacephala (Diptera: Calliphoridae))
}

Joshua Smith

Florida International University, jsmit190@fiu.edu

DOI: $10.25148 /$ etd.FIDC001174

Follow this and additional works at: https:// digitalcommons.fiu.edu/etd

Part of the Biology Commons

\section{Recommended Citation}

Smith, Joshua, "A Morphological, Functional, and Genetic Investigation of the Male Compound Eye Phenotype of Chrysomya megacephala (Diptera: Calliphoridae))" (2016). FIU Electronic Theses and Dissertations. 3060.

https://digitalcommons.fiu.edu/etd/3060 


\title{
FLORIDA INTERNATIONAL UNIVERSITY
}

\author{
Miami, Florida
}

\section{A MORPHOLOGICAL, FUNCTIONAL, AND GENETIC INVESTIGATION OF THE MALE COMPOUND EYE PHENOTYPE OF CHRYSOMYA MEGACEPHALA (DIPTERA: CALLIPHORIDAE)}

\author{
A dissertation submitted in partial fulfillment of the \\ requirements for the degree of \\ DOCTOR OF PHILOSOPHY \\ in \\ BIOLOGY \\ by \\ Joshua Laurence Smith
}

2016 
To: Dean Michael R. Heithaus

College of Arts, Sciences and Education

This dissertation, written by Joshua Laurence Smith, and entitled A

Morphological, Functional, and Genetic Investigation of the Male Compound Eye Phenotype of Chrysomya megacephala (Diptera: Calliphoridae), having been approved in respect to style and intellectual content, is referred to you for judgment.

We have read this dissertation and recommend that it be approved.

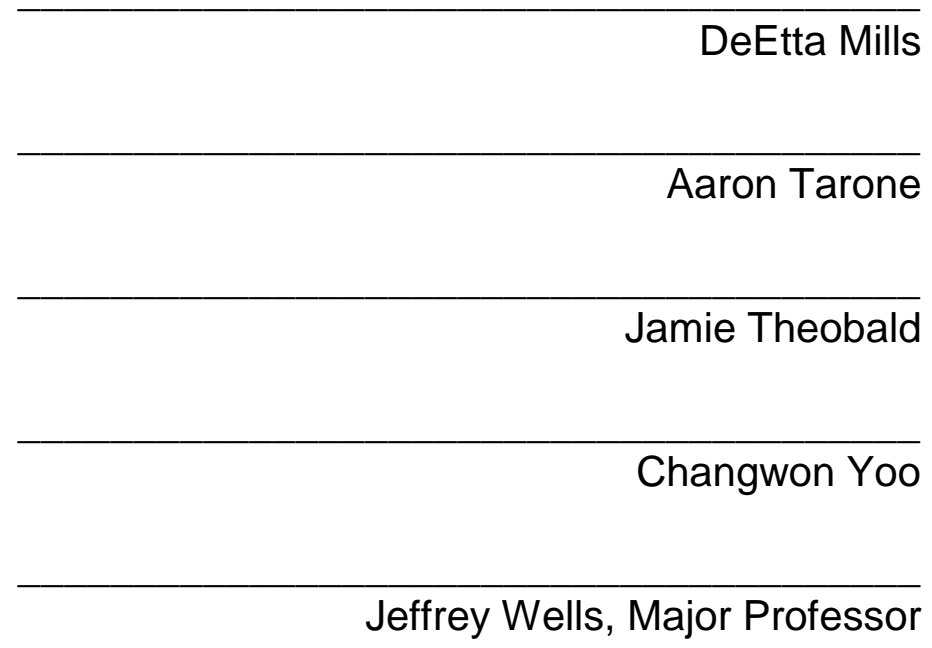

Date of Defense: October 26, 2016

The dissertation of Joshua Laurence Smith is approved.

Dean Michael R. Heithaus
College of Arts, Sciences and Education

College of Arts, Sciences and Education

Andrés G. Gil

Vice President for Research and Economic Development and Dean of the University Graduate School

Florida International University, 2016 


\section{COPYRIGHT PAGE}

Chapters II, III, and IV have been published in peer-reviewed journals and follow the formatting guidelines of the respective journals. Chapter II is published in the "Journal of Insect Science" and is open access, so it can be reproduced freely here for non-commercial purposes. Chapter III is published in "Forensic Science International" which allows for the institution awarding the degree to publicly post published work included in a dissertation as long as the appropriate DOI information is included. Chapter IV is a pre-copyedited, author-produced version of an article accepted for publication in the "Journal of Medical Entomology" following peer review. Chapters I, V, and VI have been formatted following the guidelines for publications from the Entomological Society of America.

\section{CHAPTER II}

Smith J.L., N.A. Palermo, J.C. Theobald, and J.D. Wells. 2015. Body size, rather than male eye allometry, explains Chrysomya megacephala (Diptera: Calliphoridae) activity in low light. J. Insect Sci. 15(1): 133.

\section{CHAPTER III}

Smith, J.L., N.A. Palermo, J.C. Theobald, and J.D. Wells. 2016. The forensically important blow fly, Chrysomya megacephala (Diptera: Calliphoridae), is more likely to walk than fly to carrion at low light levels. Forensic Sci. Int. 266: 245-249. DOI: http://dx.doi.org/10.1016/j.forsciint.2016.06.004

\section{CHAPTER IV}

Smith, J.L., and J.D. Wells. In press. Isolation of the male-specific transformer exon as method for immature specimen sex identification in Chrysomya megacephala (Diptera: Calliphoridae). J. Med. Entomol. DOI: 10.1093/jme/tjw198

All other materials ( $@$ Copyright 2016 by Joshua Laurence Smith

All rights reserved. 


\section{DEDICATION}

To Cristin, for everything. 


\section{ACKNOWLEDGMENTS}

I would like to thank my advisor, Dr. Jeffrey Wells, for accepting me in his lab and giving me the guidance required to develop as an independent scientist.

In addition to their time and guidance as participating members of my committee, I would also like to thank Dr. Jamie Theobald for allowing me to use his equipment, lab space, and introducing me to the world of insect behavior; Dr. DeEtta Mills for use of her lab space during my first year and for guidance in matters pertaining to my role as Genetics Head TA; Dr. Changwon Yoo for offering statistical advice when needed; and Dr. Aaron Tarone for being willing to go through the headaches associated with Skyping in for meetings and attending my qualifying exam in person. I would also like to thank Fanchen Bao, Nicholas Palermo, Carlos Ruiz, Monica Rodriguez-Silva, and Dr. Ivan Jozic for their help and friendship throughout my time at Florida International University.

I also need to thank my family for their love and support. My mom has always been there to share in my success and support me through the failures.

Finally, and most importantly, I want to thank my wife, Cristin, for unlimited patience and love. The pursuit of a Ph.D. is a very selfish endeavor, as long hours and undivided attention go towards the completion of a goal that can seem very open ended. All I can say, Cristin, is I am grateful every day for you. 
ABSTRACT OF THE DISSERTATION

A MORPHOLOGICAL, FUNCTIONAL, AND GENETIC INVESTIGATION OF

THE MALE COMPOUND EYE PHENOTYPE OF CHRYSOMYA

MEGACEPHALA (DIPTERA: CALLIPHORIDAE)

by

Joshua Laurence Smith

Florida International University, 2016

Miami, Florida

Professor Jeffrey Wells, Major Professor

A very unique compound eye in dipterans is found in males of the forensically important blow fly, Chrysomya megacephala (Diptera: Calliphoridae). This compound eye is characterized by an area of enlarged dorsal facets that, unlike almost all other regional changes in dipteran ommatidia size, is not accompanied by a change in resolution. This region is believed to play a role in mate tracking and allow for increased light capture, though no behavioral studies have tested these claims. An initial goal of the dissertation was to examine the function of this compound eye. Using allometric measurements coupled with behavioral tests, I found larger males had larger eyes and proportionally more dorsally enlarged facets. This finding suggested that larger individuals would move at lower light levels. When comparing similar sized male and females, however, body size and not the specialized male dorsal region dictated the light level at which movement occurred. 
A second focus of this dissertation was the development of tools to understand how the male compound eye is genetically regulated. The malespecific enlarged dorsal ommatidia offer a model for understanding how a gross morphological difference of a feature present in both sexes can arise when much of the same genetic content is shared. Since the genes regulating compound eye development are mainly expressed during stages when sex cannot be efficiently determined, I first designed a molecular test for identifying sex by amplifying a region of the transformer gene differentially spliced by sex in blow flies from other genera. Then, I was able to compare temporal patterns of gene expression for rhodopsin genes in separate sexes for the first time in blow flies, which allowed for an initial investigation into the expression patterns influencing the development of the male compound eye.

Apart from the biological significance regarding a unique compound eye, the forensic importance of $C$. megacephala means that information obtained in this dissertation can be utilized by forensic investigators. The work on low light level movement adds to the understanding of nocturnal oviposition patterns and the ability to sex immature specimens allows for the determination of sex specific development rates. 


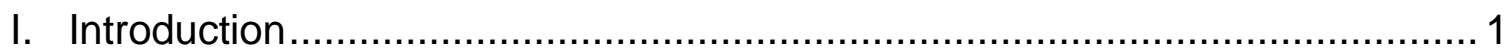

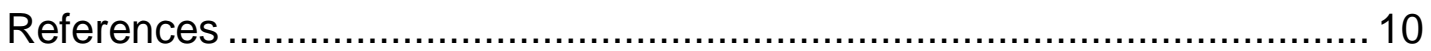

II. Body size, rather than male eye allometry, explains Chrysomya megacephala (Diptera: Calliphoridae) activity in low light................................. 14

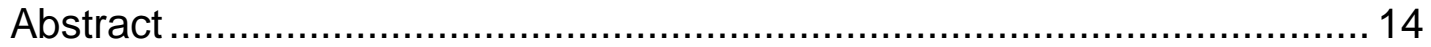

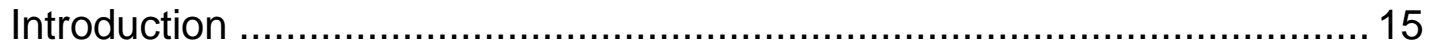

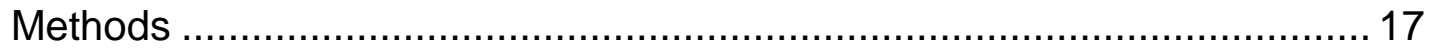

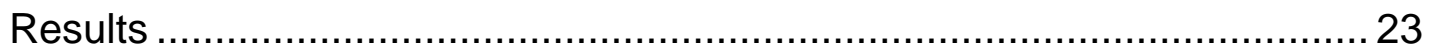

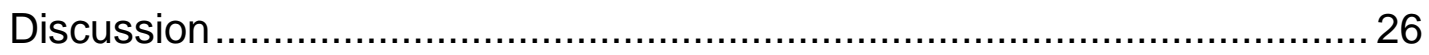

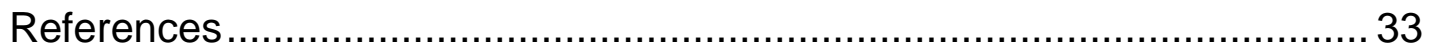

III. The forensically important blow fly, Chrysomya megacephala (Diptera: Calliphoridae), is more likely to walk than fly to carrion at low light levels ......... 45

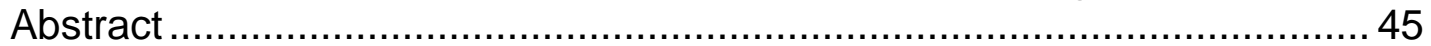

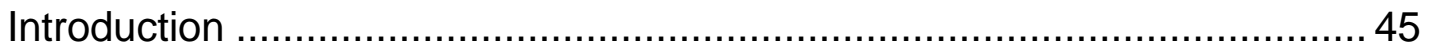

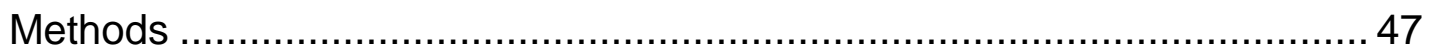

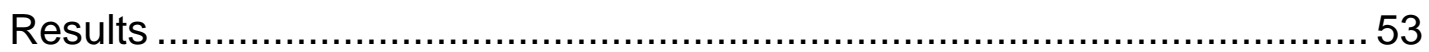

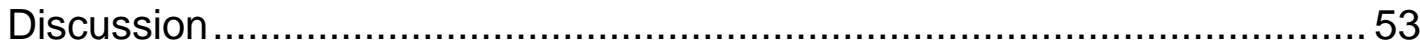

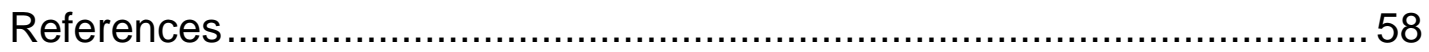

IV. Isolation of the male-specific transformer exon as a method for immature specimen sex identification in the blow fly, Chrysomya megacephala (Diptera:

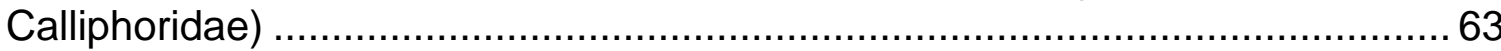

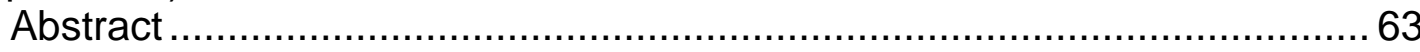

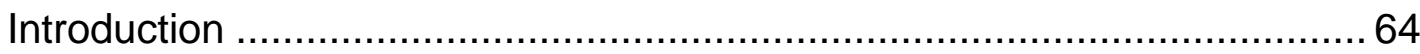

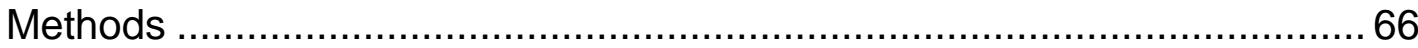

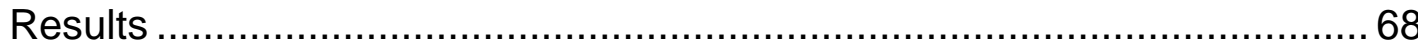

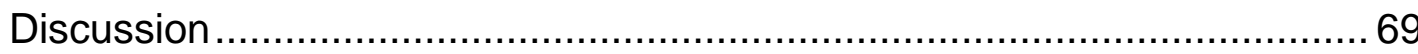

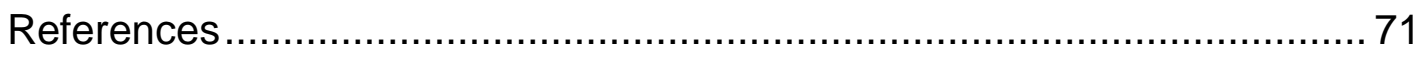

V. Expression of rhodopsin 1 and 3 in Chrysomya megacephala (Diptera: Calliphoridae): a preliminary search for candidate genes responsible for the

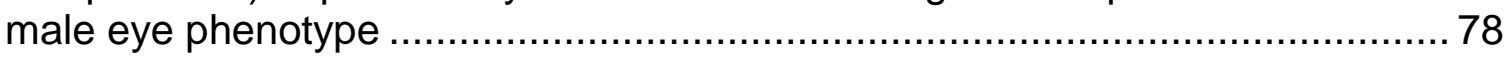

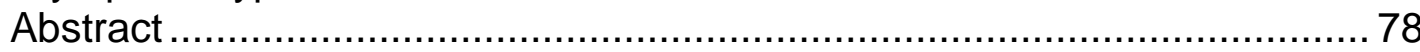

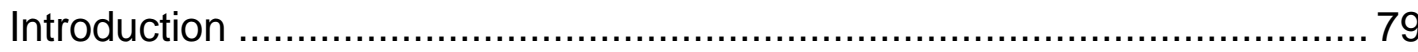

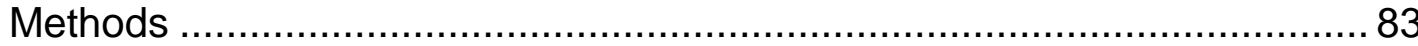

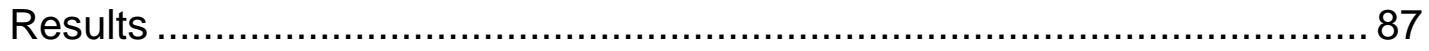

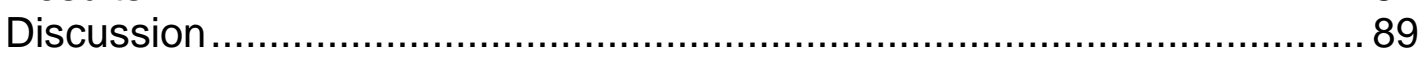

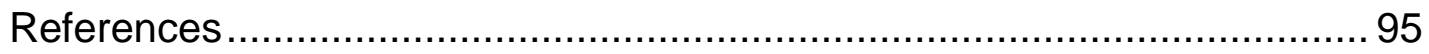




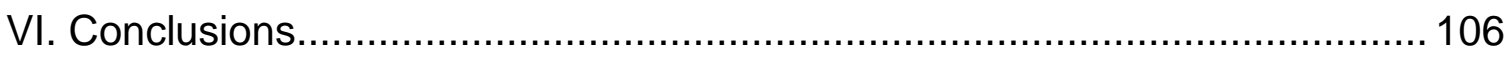

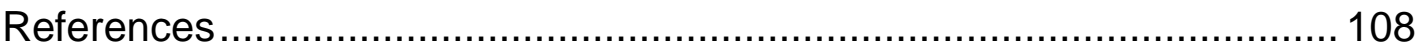

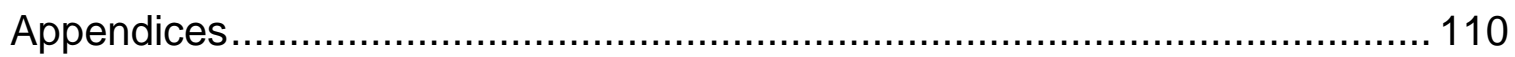

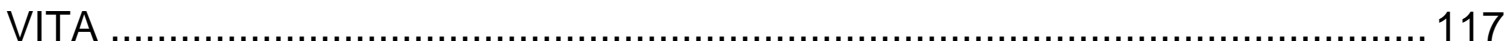




\section{LIST OF TABLES}

TABLE

PAGE

2.1 Results of regression analysis for various morphological features ( $\pm \mathrm{SE}$ ) when compared to crossvein length.

2.2 Ommatidial length ranges (minimum to maximum) for the compound eyes in a female and a male $C$. megacephala of similar size.

2.3 Results of regression analysis for light level at first movement after lights came on or last movement before lights turned off $( \pm)$ compared to crossvein length.

5.1 Summary of the $C$. megacephala individuals analyzed for each time period, including range of eye colors observed, number of females, number of males, and number of individuals that could have sex determined by eye morphology

5.2 Primers and thermal cycling conditions for the amplification of partial portions of the following genes in C. megacephala: rhodopsin 1 (rh1), rhodopsin 3 (rh3), and ribosomal protein 49 (rp49)

5.3 Summary of statistics obtained from t-tests comparing mean $C$. megacephala rh1 expression levels between males and females for each of the five time periods

A.1 Summary of gene expression as measured by band intensities for all 40 intrapuparial samples. 


\section{LIST OF FIGURES}

FIGURE

PAGE

2.1 The compound eye areas morphological measurements were taken from in Chrysomya megacephala..

2.2 The relationship between body size and different eye morphological features. 40

2.3 Behavioral analysis of $C$. megacephala daily movement.......................... 41

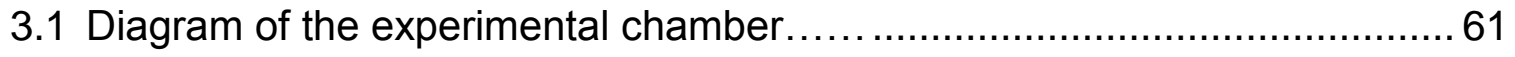

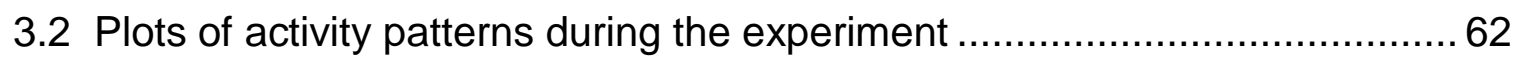

4.1 Sequence comparisons for all five blow flies for which a portion of

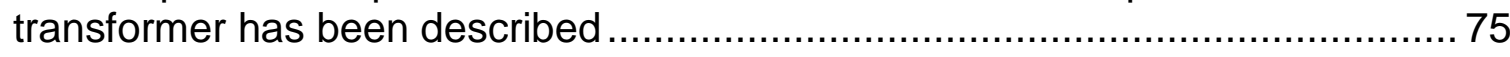

4.2 PCR yield gel images showing a male or female band pattern for (A) adults of known sex, (B) intrapuparial period specimens of known sex, (C) unknown larval specimens, and (D) egg clutches.

5.1 Amino acid alignments of rhodopsin genes in three species of blow fly ...... 99

5.2 Temporal expression of rhodopsin 1 during the late intrapuparial period .. 101

5.3 Plot of the average relative expression level of rh3 in female and male adult $C$. megacephala ( $\mathrm{n}=3$ pairs of heads for each) 102

A.1 Plots of the logarithmic transformation of various eye features in relation to body size (as described in Chapter II)

A.2 Male compound eye histology photos (100x) …................................. 111

A.3 Female compound eye histology photos (100x) .................................. 112

A.4 Histograms of the "photocell" readings when a pen or a tethered dead fly was passed through either the (a) flying or (b) walking sensor........................ 113

A.5 Comparison of (a) male and (b) female Chrysomya megacephala pharate adult compound eyes showing sexual dimophism

A.6 The DNA sequence for the male specific exon in C. megacephala. 115 


\section{ABBREVIATIONS AND ACRONYMS}

$\begin{array}{ll}\text { bp } & \text { Base pair } \\ \text { DNA } & \text { Deoxyribonucleic acid } \\ \text { IR } & \text { Infrared } \\ \text { LED } & \text { Light emitting diode } \\ \text { min } & \text { Minute } \\ \text { PCR } & \text { Polymerase chain reaction } \\ \text { RGB } & \text { Red, green, blue } \\ \text { RNA } & \text { Ribonucleic acid } \\ \text { RT-PCR } & \text { Reverse transcription polymerase chain reaction } \\ \text { SE } & \text { Standard error } \\ \text { UV } & \text { Ultraviolet }\end{array}$




\section{CHAPTER I: Introduction}

The insect compound eye is characterized by a variable number of ommatidia, similar in appearance, that cover its surface (Nilsson and Kelber 2007). The way in which the compound eye interprets information is predicated in part by the type of visual system it employs (Land and Nilsson 2002). A common visual system found in insect is the apposition compound eye. Within each ommatidium of the apposition compound eye is a fused rhabdom, which is composed of eight parts (reviewed in Agi 2014). Each of these eight parts receives input from the same point source, and in most apposition compound eyes, there is no overlap between the field of view for each ommatidium (Land and Nilsson 2002).

The visual system in found in higher flies (Muscomorpha) is a variation of the normal apposition compound eye type (Land and Nilsson 2002, reviewed in Agi 2014). Instead of having a fused rhabdom, these flies have an open rhabdom consisting of separate rhabdomeres (also called photoreceptors) (reviewed in Agi 2014). The advantage to this overall separation is that rhabdomeres within a single ommatidium all have slightly different field of view angles, with the angle of one rhabdomere being the same as a different rhabdomere in adjacent ommatidia (reviewed in Agi 2014). The signal from the rhabdomeres projects to the same portion of the brain, resulting in the signal being interpreted as a single source that is seven times as bright as would be seen in the same eye that 
employs an apposition visual system (van Hateren 1987). This sub-type of apposition eye is known as a neural superposition eye (Land and Nilsson 2002).

The model organism for compound eye structure and development in dipterans is Drosophila melanogaster. The organization of the $D$. melanogaster compound eye is highly regular, with approximately uniform hexagonal ommatidia covering the surface of the eye (Tomlinson and Ready 1987). The regularity is also present internally, as each ommatidium contains the eight photoreceptors in the same arrangement (Ready et al. 1976). This repetitive consistency is the result of a highly ordered pattern of events that begins with differential levels of gene expression as early as the first larval instar to establish polarity within the eye-antennal imaginal disc (Singh et al. 2012). The differentiation of the cells that will become the compound eye begins during the mid-third larval instar period as a wave of mitotic divisions, referred to as the morphogenetic furrow (Ready et al. 1976), sweeps across the eye-antennal imaginal disc. A precise pattern of cell signaling and sequential gene expression is carried on throughout the intrapuparial period (Cagan and Ready 1989), leading to the adult emerging with the completed compound eye.

Much of what is known about the genetic timeline of events in $D$. melanogaster compound eye development is based on the investigation of the genetic underpinnings of an abnormal phenotype, as external deficiencies are often associated with internal abnormalities. For example, the rough gene is characterized phenotypically by ommatidia that lack the smooth surface seen in the wild type (Tomlinson et al. 1988). The external appearance of the abnormal 
phenotype is internally characterized by a decreased amount of photoreceptors (Tomlinson et al. 1988).

While $D$. melanogaster provides a basic blueprint for dipteran eye structure and development because of the wealth of morphological and genetic data available, there are dipterans that deviate from this basic plan. A driver of some of these deviations is sexually dimorphic behaviors such as searching for mates (Hardie et al. 1981, O'Grady and Mclver 1987). Males of the house fly Musca domestica, for example, have a region in the dorsal portion of their eye containing enlarged facets referred to as the "love spot" (Hardie et al. 1981). Whereas the two central photoreceptors within each ommatidium typically express genes used in color discrimination, the distal most central photoreceptor in this region, $\mathrm{R} 7$, instead expresses a gene known to play a role in motion detection (Hardie et al. 1981), leading to speculation that the function of this area is to track potential mates. A different example of how motion detection can be increased for a male dipteran is present in the black fly Simulium vittatum (O'Grady and Mclver 1987). Males of this species have a dorsal area that is characterized by facets nearly twice as large as the ventral facets, but these ommatidia lack the R7 photoreceptor used to help color discrimination (O'Grady and Mclver 1987). This indicates that color detection is less important in this region believed to be specialized for mate tracking (O'Grady and Mclver 1987).

Deviations from the regular structure of ommatidium seen in $D$. melanogaster are interesting from two perspectives. Morphologically, structural differences can be representative of different functional properties of the eye 
design. Genetically, deviations can lead to insights as to how different features develop. Investigations into finer genetic differences are more feasible now as more draft genomes and transcriptomes of non-model dipterans are becoming available (Sze et al. 2012, Gilchrist et al. 2014, Scott et al. 2014a, Wang et al. 2015, Anstead et al. 2015).

A compound eye that provides an interesting model to study both visual morphological adaptation and genetic regulation is the male compound eye of the blow fly Chrysomya megacephala (F.). Morphologically, this eye is relatively unique as males have dorsal ommatidia that are upwards of four times as large as their female counterparts (Kurahashi 1982, van Hateren et al. 1989). This change in size is not accompanied by a change in resolution, as is common in external facet size changes, resulting in facets that only seem to increase sensitivity through increased light capture (van Hateren et al. 1989). This type of region has only been described in one other dipteran, the hoverfly Eristalis tenax (Straw et al. 2006). Some Chrysomya that are closely related to C. megacephala show varying degrees of this same dimorphism (Singh 2011), though their internal morphology has not been investigated, probably because each has a more limited distribution and less economic importance compared to $C$. megacephala (Wells et al. 1994). The adaptive value of this unusual compound eye has not been demonstrated, however plausible advantages are: 1) increase light capture, which would increase movement at lower light levels; and 2) to see further in areas of higher luminance (van Hateren et al. 1989). 
Another interesting morphological question is whether the dorsally enlarged facets of male $C$. megacephala provide an example of a trait that grows disproportionate to body size. Investigations regarding how a trait scales in relation to body size are important for understanding the level of phenotypic plasticity that exists for the trait (Shingleton et al. 2007). In particular, traits that increase the ability to find and capture mates can often scale in a non-isometric fashion (Emlen and Nijhout 2000). It is not known how the specialized dorsal facets of the male C. megacephala compound eye grow in proportion to body size, as previous studies did not consider body size when measuring compound eye features (van Hateren et al. 1989, Stavenga et al. 1990, Sukontsaon et al. 2008). For C. megacephala, a holometabolous species dependent on carrion for larval development, the prospect of food running out is a very real possibility that leads to size variation in adults. This lifestyle makes the study of how traits scale in relation to body size particularly relevant for this species.

From a genetic standpoint, this eye is interesting because it is a gross morphological difference between the very recently diverged sister species $C$. megacephala and C. pacifica (Singh and Wells 2011). This difference was first thought to be evidence for the recent and rapid evolution of this eye dimorphism (along with adaptation to human-altered environments), because C. pacifica has the uniform facet size characteristic of most higher flies (Kurahashi 1982, 1991). Subsequent phylogenetic analysis, however, supports the hypothesis that relative to the $C$. megacephala lineage of the genus Chrysomya, it is the $C$. pacifica eye that most recently evolved (Singh and Wells 2011). While the eye 
represents a gross morphological difference, the two species can interbreed in the lab, and resulting offspring had an intermediate eye phenotype (J. Wells, personal communication). This ability to interbreed suggests that much of the genetic material in these species is shared, and the morphological differences in the male eye are relatively new events that led to their speciation from one another.

Apart from investigating differences between two different species, the compound eye of $C$. megacephala also provides a model for understanding how sexually dimorphic differences can arise within the same species. An example of a sexually selected trait in Dipterans is the sex comb found in some male species of Drosophila, which can show great phenotypic variance between closely related species and is absent in females (Tanaka et al. 2011). It is proposed that differences in sex comb morphology are associated with differential levels of expression for genes in the sex determination pathway (Tanaka et al. 2011). As the male compound eye in $C$. megacephala is believed to play a role in mate tracking, it is very likely this feature is under sexual selection and genes involved in the sex determination pathway undoubtedly have a role to play in the genetic regulation of the compound eye.

In addition to being an interesting model for studying the morphology and function of a unique eye type and the genetic regulation of a sexually dimorphic trait that is grossly different between two closely related species the carrion feeding behavior of $C$. megacephala makes it important to forensic entomologists (Wells and Kurahashi 1994). Chrysomya megacephala is widespread (Wells 
1991), so in many areas it can be of forensic utility to investigators attempting to estimate the minimum time since death by aging immature entomological specimens developing on a corpse (Catts and Goff 1992).

The purpose of this doctoral dissertation is to investigate how the male eye morphology observed in $C$. megacephala influences low light level behavior and to develop tools that could be used to help determine how the derived eye phenotype is genetically regulated. Also, the role of $C$. megacephala as an insect of forensic importance allows for portions of this work to have direct applications in the field of forensic entomology.

Chapter II (published as Smith et al. 2015) determines how the eye changes as a function of body size. Since the enlarged dorsal eye morphology is only found in males, it is believed to play a role in mating. It is not unusual that morphological traits can scale disproportionately when associated with sexual selection (Emlen 1994). This chapter compared how different eye features scale with body size, and used this information to devise a hypothesis concerning the relationship between eye function and size. The hypothesis was tested using a custom built motion detector that enabled recording low light level walking movement. On a basic level, this chapter reveals how a relatively unique sexually dimorphic trait changes with body size and how size changes can alter behavior. On a more applied level, a better understanding of how a carrion fly responds to a corpse during low light conditions is of interest to forensic entomologists.

Chapter III (published as Smith et al. 2016) investigates the role the male C. megacephala compound eye morphology plays in low light level flight, a factor 
not considered in Chapter II. By using a more elaborate custom built sensor, low light level walking and flying were directly compared as were differences in movement based on sex. A rotten meat source was also incorporated, giving the flies an external stimulus to prompt movement that was absent in previous work (Smith et al. 2015). On a basic level this tested whether the male specific eye morphology would allow for low light level flight. This work has direct applications to the field of forensic entomology, as the likelihood of oviposition under nocturnal light levels is a factor that is debated (for example compare results in Greenberg 1990, Singh and Bharti 2001, to those in Stamper and Debry 2007, Amendt et al. 2008, Stamper et al. 2009) and influences estimates of the minimum time since death.

Chapter IV (Smith and Wells In press) describes the first molecular tool for determining sex in the larval and intrapuparial stages of $C$. megacephala, developmental periods when many of the genes involved in patterning and retinal development are expressed. Apart from karyotyping (Azeredo-Espin and Pavan 1983, Parise-Maltempi et al. 2001), which is known to be difficult in insects (Popescu and Dutrillaux 2001), there was no way to identify sex in $C$. megacephala during these stages. With recently acquired next generation sequencing data, the male specific transformer exon for $C$. megacephala was isolated as has been done for blow flies in other genera (Scott et al. 2014b) and a RT-PCR based test was developed for immature specimen sex identification. On a basic level, this work demonstrates a certain level of conservation exists in the sex determination system across three different genera of blow flies. An 
application of this work is that sexually specific differences in development rate and gene expression can now be explored in C. megacephala.

In Chapter V, the sexually dimorphic expression of rhodopsin 1 (rh1) and 3 (rh3) is investigated. These later expressing intrapuparial period genes have been tied to either specialized areas of enlarged male facets in other species (Hardie et al. 1981) or to increases in dorsal ommatidial size in adults (Posnien et al. 2012). The expression level of $r h 1$ for males and females during the later stages of C. megacephala intrapuparial development was investigated separately in an effort to determine if sexually dimorphic differences in the timing of expression for this gene may play a role in the unique male compound eye phenotype. Similarly, the expression level of $r h 3$ was compared in adult males and females to determine if previous correlations of increased $r h 3$ expression and dorsal enlargement remained consistent for C. megacephala. In addition to an exploration of genes that may contribute to the male compound eye phenotype, on a more applied level this work represents the first investigation of sex specific blow fly intrapuparial gene expression. Previous work has shown sex can alter growth rates (Picard et al. 2013), and gene expression is used to estimate the age of blow fly intrapuparial specimens (Tarone and Foran 2011, Boehme et al. 2013), so understanding how gene expression can vary by sex at different times can aid forensic entomologists in constructing more accurate models of the specimen age used to estimate the time since death. 


\section{References}

Agi, E., M. Langen, S.J. Altschuler, L.F. Wu, T. Zimmermann, and P.R. Hiesinger. 2014. The evolution of development and neural superposition. J. Neurogenetics 28: 216-232.

Amendt, J., R. Zehner, and F. Reckel. 2008. The nocturnal oviposition behavior of blowflies (Diptera: Calliphoridae) in Central Europe and its forensic implications. Forensic Sci. Int. 175: 61-64.

Anstead, C.A., P.K. Korhonen, N.D. Young, R.S. Hall, A.R. Jex, S.C. Murali, D.S.T. Hughes, S.F. Lee, T. Perry, A.J. Stroehlein, B.R.E. Ansell, B. Breugelmans, A. Hofmann, J. Qu, S. Duggan, S.L. Lee, H. Chao, H. Dinh, Y. Han, H.V. Doddapaneni, K.C. Worley, D.M. Muzny, P. Ioannidis, R.M. Waterhouse, E.M. Zdobnov, P.J. James, N.H. Bagnall, A.C. Kotze, R.A. Gibbs, S. Richards, P. Batterham, and R.B. Gasser. 2015. Lucilia cuprina genome unlocks parasitic fly biology to underpin future interventions. Nat. Comm. 6:7344.

Azeredo-Espin, A.M.L., and C. Pavan. 1983. Karyotypes and possible regions of origin of three species of Calliphoridae (Diptera) recently introduced in Brazil. Rev. Bras. Genet. 4: 619-638.

Cagan, R.L., and D.F. Ready. 1989. The emergence of order in the Drosophila pupal retina. Dev. Bio. 136: 346-362.

Catts, E.P., and M.L. Goff. 1992. Forensic entomology in criminal investigations. Annu. Rev. Entomol. 37: 253-272.

Emlen, D.J. 1994. Environmental control of horn length dimorphism in the beetle Onthophagous acuminatus (Coleoptera: Scarabaedae). Proc. R. Soc. Lond. B. 256: 131-136.

Emlen, D.J. and H.F. Nijhout. 2000. The development and evolution of exaggerated morphologies in insects. Annu. Rev. Entomol. 45: 661-708.

Gilchrist, A.S., D.C.A Shearman, M. Frommer, K.A. Raphael, N.P. Deshpande, M.R. Wilkins, W.B. Sherwin, and J.A. Sved. 2014. The draft genome of the pest tephritid fruit fly Bactrocera tryoni: resources for the genomic analysis of hybridizing species. BMC Genomics 15: 1153.

Greenberg, B. 1990. Nocturnal oviposition behavior of blow flies (Diptera: Calliphoridae). J. Med. Entomol. 27: 807-810. 
Hardie, R.C., N. Franceschini, W. Ribi, and K. Kirschfeld. 1981. Distribution and properties of sex-specific photoreceptors in the fly Musca domestica. J. Comp. Phys. 145: 139-152.

Kurahashi, H. 1982. Possible origin of a synanthropic fly Chrysomya megacephala in New Guinea (Diptera: Calliphoridae). Monographiae Biologicae. 42: 689-698.

Kurahashi, H. 1991. Blow flies from Samoa with a description of a new species of Chrysomya (Diptera, Calliphoridae). Jp. Jn. Ent. 59: 627-636.

Land, M.F., and D-E. Nilsson. 2012. Apposition compound eye. In Animal Eyes. Oxford University Press, Inc., New York, NY.

Nilsson, D-E., and A. Kelber. 2007. A functional analysis of compound eye evolution. Arthropod Struct. Dev. 36: 373-385.

O'Grady, G.E., and S.B. Mclver. 1987. Fine structure of the compound eye of the black fly Simulium vittatum (Diptera: Simuliidae). Can. J. Zoo. 65: 1454-1469.

Parise-Maltempi, P.P., and R.M.P Avancini. 2001. C-banding and FISH in chromosomes of the blow flies Chrysomya megacephala and Chrysomya putoria (Diptera, Calliphoridae). Mem. Inst. Oswaldo Cruz 96: 371-377.

Popescu, P., and B. Dutrillaux. 2000. Preparation of chromosome spreads, pp.11-17. In P. Popescu, H. Hayes, and B. Dutrillaux (eds.), Techniques in Animal Cytogenetics. Springer-Verlag, Berlin.

Ready, D.F., T.E. Hanson, and S. Benzer. 1976. Development of the Drosophila retina, a neurocrystalline lattice. Dev. Bio. 53: 217-240.

Scott, J.G., W.C. Warren, L.W. Beukeboom, D. Bopp, A.G. Clark, S.D. Giers, M. Hediger, A.K. Jones, S. Kasai, C.A. Leichter, M. Li, R.P. Meisel, P. Minx, T.D. Murphy, D.R. Nelson, W.R. Reid, F.D. Rinkevich, H.M. Robertson, T.B. Sackton, D.B. Satelle, F, Thibauld-Nissen, C. Tomlinson, L. van de Zande, K.K.O. Walden, R.K. Wilson, and N. Liu. 2014a. Genome of the house fly, Musca domestica L., a global vector of diseases with adaptations to a septic environment. Genome Biol. 15: 466.

Scott, M.J., M.L. Pimsler, and A.M. Tarone. 2014b. Sex determination mechanisms in the Calliphoridae (Blow Flies). Sex. Dev. 8: 29-37. 
Shingleton, A.W., W.A. Frankino, T. Flatt, H.F. Nijhout, and D.J. Emlen. 2007. Size and shape: the developmental regulation of static allometry in insects. BioEssays 29: 536-548.

Singh, D., and M. Bharti. 2001. Further observations on the nocturnal oviposition behavior of blow flies (Diptera: Calliphoridae). Forensic Sci. Int. 120: $124-126$.

Singh, A., M. Tare, O.R. Puli, and M. Kango-Singh. 2012. A glimpse in dorsoventral patterning of the Drosophila eye. Dev. Dynam. 241: 69-84.

Singh, B. 2011. Molecular phylogeny of the Oestroidea. Ph.D. Dissertation, West Virginia University, Morgantown.

Smith J.L., N.A. Palermo, J.C. Theobald, and J.D. Wells. 2015. Body size, rather than male eye allometry, explains Chrysomya megacephala (Diptera: Calliphoridae) activity in low light. J. Insect Sci. 15(1): 133.

Smith, J.L., N.A. Palermo, J.C. Theobald, and J.D. Wells. 2016. The forensically important blow fly, Chrysomya megacephala (Diptera: Calliphoridae), is more likely to walk than fly to carrion at low light levels. Forensic Sci. Int. 266: 245-249.

Smith, J.L., and J.D. Wells. In press. Isolation of the male-specific transformer exon as a method for immature specimen sex identification in Chrysomya megacephala (Diptera: Calliphoridae). J. Med. Entomol. DOI: 10.1093/jme/tjw198.

Stamper, T., and R.W. Debry. 2007. The nocturnal oviposition behavior of carrion flies in rural and urban environments: methodological problems and forensic implications. Can. Soc. of Forensic Sci. J. 40: 173-182.

Stamper T., P. Davis, and R.W. Debry. 2009. The nocturnal ovipositing behavior of carrion flies in Cincinnati, Ohio. J. Forensic Sci. 54: 14501452.

Stavenga, D.G., R. Kruizinga, and H.L Leertouwer. 1990. Dioptrics of the facet lenses of male blow flies Calliphora and Chrysomia. J. Comp. Physiol. A. 166: 365-371.

Straw, A.D., E.J. Warrant, and D.C. O'Carroll. 2006. A 'bright zone' in male hoverfly (Eristalis tenax) eyes and associated faster motion detection and increased contrast sensitivity. J. Exp. Biol. 209: 4339-4354. 
Sukontason, Ka., T. Chaiwong, S. Piangjai, S. Upakut, K. Moophayak, and Ko. Sukontason. 2008. Ommatidia of blow fly, house fly, and flesh fly: implication of their vision efficiency. Parasitol. Res. 103: 123-131.

Sze, H.S., J.P. Dunham, B. Carey, P.L. Chang, F. Li, R.M. Edman, C. Fjeldsted, M.J. Scott, S.V Nuzhdin, and A.M. Tarone. 2012. A de novo transcriptome assembly of Lucilia sericata (Diptera: Calliphoridae) with predicted alternative splices, single nucleotide polymorphisms, and transcript expression estimates. Insect Mol. Bio. 21: 205-221.

Tanaka, K., O. Barmina, L.E. Sanders, M.N. Arbeitmen, and A. Kopp. 2011. Evolution of sex-specific traits through changes in HOX-Dependent doublesex expression. PLoS Biology 9: e1001131.

Tomlinson, A., and D.F. Ready. 1987. Neuronal differentiation in the Drosophila ommatidium. Dev. Bio. 120: 366-376.

Tomlinson, A., B.E. Kimmel, and G.M. Rubin. 1988. Rough, a Drosophila homeobox gene required in photoreceptors $\mathrm{R} 2$ and $\mathrm{R} 5$ for inductive interactions in the developing eye. Cell 55: 771-784.

van Hateren, J.H. 1987. Neural superposition and oscillations in the eye of the blowfly. J. Comp. Physiol. A 161: 849-855.

van Hateren, J.H., R.C. Hardie, A. Rudolph, S.B. Laughlin, and D.G. Stavenga. 1989. The bright zone, a specialized dorsal eye region in the male blow fly Chrysomia megacephala. J. Comp. Physiol. A 164: 297-308.

Wang, X., M. Xiong C. Lei, and F. Zhu. 2015. The developmental transcriptome of synanthropic fly Chrysomya megacephala and insights into olfactory proteins. BMC Genomics 16: 20.

Wells, J.D. 1991. Chrysomya megacephala (Diptera: Calliphoridae) has reached the continental United States: review of its biology, pest status, and spread around the world. J. Med. Entomol. 28: 471-473.

Wells, J.D., and H. Kurahashi. 1994. Chrysomya megacephala development: Rate, variation and the implications for forensic entomology. Jpn. J. Sanit. Zool. 45: 303-309.

Wells, J.D., M.M. Singh, K. Suzuki, M. Miura \& H. Kurahashi. 1994. Male eye dimorphism and synanthropy in Chrysomya pinguis (Diptera: Calliphoridae). Jpn. J. Sanit. Zool. 45: 297-300. 


\title{
CHAPTER II: Body size, rather than male eye allometry, explains Chrysomya megacephala (Diptera: Calliphoridae) activity in low light
}

\begin{abstract}
Male Chrysomya megacephala (F.) blow fly compound eyes contain an unusual area of enlarged dorsal facets believed to allow for increased light capture. This region is absent in females and has been hypothesized to aid in mate tracking in low light conditions and/or at greater distances. Many traits used in the attraction and capture of mates are allometric, growing at different rates relative to body size. Previous reports concerning C. megacephala eye properties did not include measurements of body size, making the relationship between the specialized eye region and body size unclear. We examined different morphological features of the eye among individuals of varying sizes. We found total eye size scaled proportionately to body size, but the number of enlarged dorsal facets increased as body size increased. This demonstrated that larger males have an eye that is morphologically different than smaller males. Based on external morphology, we hypothesized that since larger males have larger and a greater number of dorsally enlarged facets, and these facets are believed to allow for increased light capture, larger males would be active in lower light levels than smaller males and females of equal size. In a laboratory setting, larger males were observed to become active earlier in the morning than smaller males, although they did not remain active later in the evening. However, females followed the same pattern at similar light levels suggesting that overall
\end{abstract}


body size rather than specialized male eye morphology is responsible for increased activity under low light conditions.

\section{Introduction}

Traits that aid in the capture or attraction of mates can often grow disproportionately in relation to body size among holometabolous insects (reviewed in Emlen and Nijhout 2000). The size of these traits is generally nutrition dependent, as the exaggerated features are not necessary to complete development and can be notably absent in smaller individuals (Emlen 1994). Since the adult size of holometabolous insects is determined by larval feeding (Shingleton et al. 2007), to develop a larger trait as an adult, a larva must feed beyond the minimum requirement to complete metamorphosis (Emlen et al. 2007). This presents a life history trade off in that achieving larger size requires individuals to remain for longer in what may be a relatively vulnerable larval stage (Hanski 1987).

Blow flies are examples of holometabolous insects whose adult size is dependent on larval feeding. Many blow flies feed on carrion (Norris 1965, Hanski 1987, Erzinçlioglu 1996). The use of such a temporary and finite food source leads to the possibility that individuals will not be able to feed long enough to complete development due to intense competition that results in the loss of the food source (Norris 1965, Hanski 1987). Furthermore, blow fly larvae are relatively defenseless against direct predation (Faria et al. 1999) and vertebrate scavenging (Reeves 2009). Once a blow fly has obtained enough nutrition it can move off the food source, pupate, and become a relatively small adult, or stay on 
the food and continue feeding, risking predation while possibly becoming a larger adult. A size increase that elevates the performance of a trait used to find mates might generate selection to stay on a food source for longer.

An example of a sexually dimorphic trait that has been hypothesized to help locate mates in a blow fly is the male compound eye of Chrysomya megacephala (F.). Adult males possess a dorsal area of ommatidia that are drastically larger than the facets in the ventral region (Kurahashi 1982; Figure 2.1a). Females of the same species do not have this area of dorsal enlargement (Sukontason et al. 2008; Figure 2.1a). While a compound insect eye containing distinct regions of facet size is not uncommon (reviewed in Land 1997), it is very rare in muscomorph flies, and even more unusual is that the increase in facet size is not accompanied by a change in resolution (van Hateren et al. 1989). This has led to the dorsal area being referred to as the "bright zone" since the enlarged facets are believed to be used for increased light capture, perhaps allowing males to either search for females at lower light levels or from greater distances in higher light levels (van Hateren et al. 1989).

Previous morphological publications concerning the C. megacephala male eye (van Hateren et al. 1989, Stavenga et al. 1990, Sukontason et al. 2008) did not investigate the relationship between body size and eye shape. Our first objective was to determine how eye morphology scales with body size in male $C$. megacephala. Our second objective was to analyze the behavioral implications of any variation in eye morphology in order to gain insight from a visual 
perspective as to why a blow fly might prolong the risky larval phase of its life cycle in order to increase its adult body size.

\section{Methods}

Study Groups for Eye Morphology. Chrysomya megacephala with four sampling histories were used: 1 ) adult flies caught at a decayed meat bait near the Florida International University campus (referred to as "FIU wild"; 2) adult flies collected in a similar manner in the Florida Keys ("Keys wild"); 3) the adult offspring from a single egg clutch obtained from a laboratory colony originating from Florida International University ("FIU colony"); and 4) the adult offspring from a single egg clutch from a single female captured in Marathon, FL ("Marathon female"). The wild caught flies were all placed in laboratory cages and provided water and sugar for a period of four days after capture. At the end of the four day period, the flies were killed by freezing. From the two groups of the single egg clutches, some larvae were manipulated to generate a range of adult sizes. In order to generate small flies, between 25-30 larvae from each group were removed from the meat during the early third instar period and placed directly into sawdust where they could pupate. The remaining larvae were provided with chicken liver until each ceased feeding on its own and then pupated in sawdust. Following pupation, the newly emerged adults were allowed four days to fully mature before being killed by freezing.

Adult males from all four groups were pinned and assigned a specimen number. We generated a random permutation of the specimen numbers, and made measurements of the first 30 specimens on that list. If less than 30 
individuals were present, then all individuals were used. If damaged, individuals were omitted from that measurement. As a control for body size independent of male eye morphology, we also pinned adult females from the single egg clutch obtained from Marathon, Florida. We chose 20 females in the same manner as the males.

Measuring Fly Size and Eye Morphology. Crossvein length (dm-cu) was used as an indicator of body size (Ireland and Turner 2006). We mounted wings on microscope slides and photographed them at $25 x$ magnification. Measurements were taken by analyzing photos in Image $\mathrm{J}$ (version 1.47; National Institutes of Health; Bethesda, MD, USA). The suitability of crossvein length as an indicator of body size was determined by measuring thorax length (Jander and Jander 2002, Kelber et al. 2006) using a caliper in a subset of males from all four groups. Linear regression was used to characterize the relationship between thorax length and crossvein length.

Eye size was defined as the distance from top of the medial area of the eye to the bottom of the distal area (black lines in Fig. 2.1a). Eyes were photographed at $60 x$ magnification and the images were viewed and measured using Image J software.

To measure various aspects of eye morphology, casts were made of the eyes using clear nail polish and flattened onto a slide as described in Ribi et al. (1989). We viewed the slides under 100x magnification and photographed the areas of interest. The photos were viewed and measured using Image $\mathrm{J}$ software. 
We designated morphological references points to define the facets that were measured across different individuals (Figs. 2.1b-c). For males, one morphological landmark was the boundary separating the enlarged dorsal ommatidia from the smaller ventral ommatidia. This boundary consists of two rows containing predominantly non-hexagonal shaped ommatidia near the medial portion of the face. Within the ventral most of these two rows, we located the tenth ommatidium from the edge (Fig. 2.1b) and then measured three consecutive facets beginning with the third row distant from that point in the ventral direction (small triangles in Fig. 2.1b). From the same reference point, three consecutive facets were counted beginning with the fifth row distant in the dorsal direction (small circles in Fig. 2.1b).

For the female adults, there is not a sharp change in facet shape so an approximate reference point was determined using the medial boundary of the compound eye. The medial portion of the compound eye runs perpendicular to the longitudinal axis of the body, and then curves in the ventral half of the eye. The point approximately above this transition was used a reference (black arrow in Fig. 2.1a). From this point, we counted over three ommatidia and measured the width of two consecutive facets (triangles in Fig. 2.1c).

For the adult males, we were interested in the number of enlarged dorsal ommatidia that spanned the dorsal region. Starting with the most medial enlarged dorsal facet near the line of demarcation, the number of consecutive ommatidia in a row were counted up the dorsal area until another line of non-hexagonal shaped facets were met (dotted line in Fig. 2.1d). We used linear regression to 
characterize the relationship between the various eye features and crossvein length for both males and females.

We also performed a logarithmic transformation on the data for eye size, facet size, and facet number (not shown). By modelling allometry (as reviewed in Shingleton et al. 2007) using the formula $y=a x^{b}$, (where $x$ is body size, $y$ is the size of the trait of interest, and both $a$ and $b$ are constants), a logarithmic transformation yields a linear equation $(\log (y)=\log (a)+b \log (x))$ where the slope of the line, $b$, quantitatively describes change in trait size relative to body size. We calculated the value of $b$ to determine the rate of growth for each eye feature relative to body size for eye size, facet size, and facet number using this equation.

Histology. Individuals used for histological work were obtained from a $C$. megacephala laboratory colony established from individuals captured in Miami, FL, in June 2013. Longitudinal cross-sections of compound eyes from a single male and female of approximately equal size were obtained following the methodology described in Meyer-Rochow and Lau (2008). Histological sections were photographed and measurements of the ommatidial lengths were taken using ImageJ software. In order to get a range of ommatidial lengths, measurements for the female compound eye were taken from the upper, middle, and lower area of the histological sections. Measurements for the male compound eye were taken from upper region of the dorsal area, from near the equator in the dorsal area, from near the equator in the ventral area, and in the 
lower portion of the ventral area of the histological sections. Body size for these individuals was measured using crossvein length as previously described.

Behavior. Mate detection and capture under low light conditions requires general movement. To determine fly activity during a simulated light/dark cycle incorporating low light conditions, we used an interruption of an infrared beam caused by a moving fly within a confined space (Joshi 1999). Single flies were isolated in a translucent plastic tube lined with foam to limit the range of motion for the fly to the area with the infrared beam. Inside the tube, a moistened cotton ball was placed on one side and sugar was placed on the other (Fig. 2.3a). The apparatus included a pair of these confinement tubes, each placed between an infrared emitter and receiver. Black poster board visually separated the two tubes so that flies simultaneously placed individually in each tube could not see each other. A custom python script recorded the time, number, and photocell reading of each beam interruption.

The movement sensors were placed in a DigiTherm Incubator (Tritech Research Inc., Los Angeles, CA, USA) outfitted with a strip of LED lights. The lights were controlled by a microcontroller (Arduino UNO, Italy) programmed to simulate a light cycle with natural, gradual transitions. The simulated day cycle consisted of a period of 12 hours of darkness, followed by a 2 hour increase to a maximum level, a hold at the maximum level for 8 hours, followed by a 2 hour decrease until the lights went off.

Subjects were from what appeared to be a single egg clutch (based on egg number and arrangement, Wells and Kurahashi 1994). This was done to 
reduce genetic (Williams and Kokkinn 2005) and eliminate age related (Gibbert et al. 2001, Koh et al. 2006) effects on behavior, as only sibling pairs of equal age were used in the behavior experiments. Individuals within the clutch were reared under the same conditions, except some feeding larvae were removed early to generate smaller individuals. Large and small C. megacephala males from the same egg clutch were placed in the incubator to entrain for at least three days at the simulated day light cycle. After entrainment, a pair consisting of one large and one small male were simultaneously placed in separate tubes of the movement sensor and observed for two mornings and two evenings. We rotated the different size groups (either "large" or "small" males) between the two sensors to eliminate any bias of one sensor over the other. A total of 10 pairs consisting of a large and small male were used. For a control of the effect of body size independent of the specialized male eye morphology, this work was repeated with 10 pairs of large and small $C$. megacephala females.

Analyses were based on the time that elapsed between 1) the lights turning on and first movement of the individual, and 2) the final movement of an individual before the lights turned off. Paired t-tests were performed to determine if the differences in time were significant between body sizes. A two-sample ttest, assuming equal variance, was performed comparing the differences in time obtained on both sensors to check for any sensor bias.

The average photocell reading at first crossing after the lights came on for both days was recorded for each fly. Similarly, the average photocell reading at the last crossing in the evening before the lights went off for both days was also 
recorded for each fly. We converted the photocell readings into light levels by measuring the light level inside the incubator using a light meter (Starlite 2, Gossen, Nürnberg, Germany) at five step increments for the light program. We plotted these values against the average photocell reading across a 5000 millisecond integration time (one reading per millisecond) at the same five step increments, and used piece wise interpolation for values not directly measured. We used linear regression to characterize the relationship between the average light level and crossvein length.

Determination of Ocellus Size. For the first five pairs from both the male and female behavior experiments, we determined the width of the median and lateral ocelli by taking photos and measuring using Image $\mathrm{J}$. We used linear regression to characterize the relationship between both median ocelli and lateral ocelli width with respect to crossvein length.

\section{Results}

Crossvein Length a Suitable Indicator of Body Size. As shown in Fig. $2 a$, there is a significant positive linear relationship between crossvein and thorax length (Table 2.1). From these data it was determined that crossvein length is a suitable indicator of body size.

Eye Morphology Changes Based on Size. As shown in Fig. 2.2b, as body size increased so did the overall size of the eye in males (Table 2.1) and females (Table 2.1). Based on the results of the logarithmic regression analysis, this change is nearly isometric ( $b=0.8788$ for all males, $b=1.1115$ for females). Similarly, individual facet width also increased as body size did for the male 
dorsal (Table 2.1), male ventral (Table 2.1), and female facets (Table 2.1) (Fig. 2.2c). This rate of increase, however, was less for each of the male dorsal ( $b=$ $0.4697)$, male ventral $(b=0.5192)$, and female $(b=0.5327)$ facets compared to overall eye size. The male dorsal facets were the widest while the male ventral facets were the narrowest (Fig. 2.2c). The facet widths from the female eye, lacking a distinct size difference in dorsal and ventral facets, fell in between those of the dorsal and ventral male eye (Fig. 2.2c). For males, the ratio of the dorsal to ventral facet width was between 2.1 and 2.7 (Fig. 2.2d) and we found no significant relationship between this ratio and body size (Table 2.1).

That eye size is increasing at a higher rate than facet size increases implies that the number of ommatidia should also increase as body size does. Our observations support this as Fig. 2e shows that the number of enlarged facets in the dorsal region increased as body size increased (Table 2.1). The number of ommatidia increases at a rate similar to that of facet size $(b=0.5393$ for all males). These results showed that eye morphology changes as individuals change in size, with larger individuals having not only larger but more facets.

Based on the observed increase in ommatidia, we proposed the following hypothesis: if larger males have larger and an increased number of dorsally enlarged facets, and if these facets are used to increase light capture, then larger males should be better equipped to visually process their environment in conditions of low light than smaller males. Therefore larger males should show movement when light levels are too low for movement by smaller males. A second behavioral hypothesis was that males should be able to move in lower 
light levels than females of equal size due to the presence of the specialized region of enlarged dorsal facets.

Male Eye Properties and Population. We used four groups of males: two from different single egg clutches; and two wild caught from separate locations. Concerning total eye size, the slope for each individual group line was significantly different from zero (Table 2.1; Fig. 2.2b). The two cohorts originating from the same egg clutch had higher correlations than the wild caught individuals (Table 2.1). None of the groups were significantly different from zero for the dorsal to ventral facet width ratio. Similar to eye size, the slope for each individual group regression line did differ significantly from zero in regards to the number of ommatidia in the dorsal region (Table 2.1; Fig. 2.2e). Again a higher correlation existed among the two cohorts from single egg clutches in comparison to the wild population (Table 2.1).

Ommatidium Length. As can be seen in Table 2.2, the ranges of ommatidial lengths were similar in the male dorsal region and the female. The male ventral region had the largest maximum ommatidial length.

Behavior. To test the hypothesis of large males moving earlier in the day than small males, we measured activity at different light levels through a simulated day. As shown in Fig. 2.3b, larger males moved significantly earlier in the morning than smaller males did $(t=4.1778, \mathrm{df}=9, P=0.002384)$. Out of the twenty mornings, on only two occasions was the smaller male the first to move. In the evening, however, there was not a significant difference in movement times based on body size $(t=-0.0654, \mathrm{df}=9, P=0.9493)$. A similar pattern was 
seen in the females, with larger individuals moving earlier in the morning $(t=$ $3.8333, \mathrm{df}=9, P=0.004008$, Fig. 3b). For the females, on only four occasions out of the 20 mornings did the smaller female move before the larger one. For female movement in the morning, there was an outlier more than two and a half times the upper quartile. When removed, the difference in movement was still significant $(t=5.5835, \mathrm{df}=8, P<0.001)$ in the morning. There was not a significant difference in time during the evening $(t=-0.2290, \mathrm{df}=9, P=0.8240)$. There was no significant difference in times between the two sensors $(t=-$ $0.6920, \mathrm{df}=38, P=0.4931)$, showing no effect of the sensor.

The relationship between the average light level at first movement and body size revealed an inverse relationship in both males (Table 2.3) and females (Table 2.3, Fig. 2.3c). For both sexes, larger individuals moved at lower light levels. Comparison of males and females of similar size showed that females moved at lower average light levels (Fig. 2.3c). For the evening, however, we did not find a significant relationship between body size and light level at last movement for both males (Table 2.3) and females (Table 2.3, Fig. 2.3d).

Ocellus Size. As can be seen in Fig. 2.2f, the width of the median ocellus increased as body size increased for both males and females (Table 2.1). Similarly, the width of the lateral ocellus also increased. For both types, males have wider ocelli than females.

\section{Discussion}

\section{C. megacephala Males of Different Sizes have Morphologically}

Different Eyes. By taking into account body size, a factor absent in previous 
work on C. megacephala male eye morphology (van Hateren et al. 1989, Stavenga et al. 1990, Sukontason et al. 2008), we demonstrated that not only do eye size and ommatidium widths increase as the individual gets larger, but the number of dorsally enlarged ommatidia also increases. For an insect, enlarged facets lead to the possible undersampling of an image, since large facets use up the finite surface area of the compound eye. van Hateren et al. (1989) described two possible solutions for undersampling in $C$. megacephala: increasing the number of ommatidia or increasing the rhabdomere diameter. They found $C$. megacephala increased the rhabdomere diameter (van Hateren et al. 1989). The observations made in our current work show that larger males may also address the issue of undersampling with a larger number of ommatidia in the dorsal area.

We did not find a significant difference in dorsal to ventral facet width as body size increases. The range in dorsal to ventral facet width we observed ( 2.1-2.7) is rather less than the previous report of the dorsal facets being four times larger than the ventral facets (van Hateren et al. 1989). A reason for this may be that we measured facet widths from a different area than van Hateren et al. (1989), who did not specify exact facet location, and that facet size changes based on location. In the blow fly Calliphora vicina (=C. erythrocephala), for example, there is a change in facet size across different regions related to spatial acuity (Land and Eckhart 1985).

The relative rates of growth observed in male $C$. megacephala between body size and eye size, facet size, and ommatidia number are similar to the values reported across 15 different species of bees (Jander and Jander 2002) 
and within individuals of varying size for the same bee species (Spaethe and Chittka 2003). The similarity in results is particularly interesting considering that the previously described bee species have compound eyes containing facets with gradual changes in size, while male $C$. megacephala has a specialized region of enlarged facets sharply differentiated from those on the rest of the eye. Whether these properties would be similar in compound eyes with and without regionalization was discussed in Jander and Jander (2002), and here we provide one example where they are. Since C. megacephala does not experience a change in resolution between the enlarged dorsal facets and the smaller ventral facets, however, comparisons between our results and a compound eye with regionalization that is associated with a change in resolution should be considered.

Higher Correlation Within a Population. We found a higher correlation between body size and morphological features for the two groups originating from a single egg clutch as opposed to the two wild caught populations. This was expected as the two single egg clutch groups had similar genetic backgrounds and were reared under known environmental conditions. For the wild caught populations, however, both the genetic background and environmental conditions during development were unknown. This may explain why the female correlation values were higher than the males, since the females used in this work all came from the same egg clutch while the males consisted of both individuals from the same egg clutch and wild caught ones. These findings support the idea that studies measuring the relationship between a certain trait and body size in 
insects should use genetically similar individuals in order to observe the entire range of trait morphologies one genotype can produce when faced with different developmental environments (discussed in Emlen and Nijhout 2000).

Another factor that likely influenced the higher correlation for the groups originating from a single egg clutch is that there was less overall size variation. While in the single egg groups we were able to generate a greater size range including individuals smaller than we found in our wild caught populations, these single egg groups consisted of either very small or very large individuals. This was in contrast with the wild groups that had an overall narrower size range but more individuals that would be considered intermediate in size. Future work should consider trying to generate a gradient of sizes for the single egg groups to see if the higher correlation is still evident in comparison to a wild population.

Total Body Size, Not Specialized Male Eye Morphology, is Important for Being Active in Low Light Conditions. Our hypothesis that larger males would be able to move at lower light levels than smaller males was supported in the morning, but not in the evening. However, this same pattern of behavior was exhibited in females, who do not have the specialized dorsal region of facets. In fact, females actually moved at lower light levels than males of similar size despite having smaller overall eye sizes. For this reason, we cannot attribute the early movement in larger males to their specialized eye morphology. The difference in movement appears to be attributed to being a larger individual, and may be the result of larger individuals simply having larger eyes that capture more light (Jander and Jander 2002, Kelber et al. 2006). 
Previous work in the blow fly Calliphora vicina (=C. erythrocephala) found it took longer for activity to increase as light levels increased than it did for activity to be reduced as light levels decreased (Digby 1958). This is consistent with our current work as we observed crosses at lower light levels as the lights were decreasing in comparison to when they were increasing.

Blow flies are generally considered to be diurnal (Anderson 2001). A blow fly relies on carrion as a place to oviposit, feed, and find mates (Norris 1965, Erzinçlioglu 1996), so movement earlier in the day may confer an individual advantage because arriving first on carrion that has died during the night might help a blow fly avoid significant competition that would arise later in the day. Furthermore, by arriving early, a blow fly would have earlier access to potential mates. In the evening, however, blow flies of all sizes have had all day to visit carrion and search for mates, so staying out later is not as crucial. This suggests that being a larger blow fly is advantageous as it allows for early morning movement.

Internal Compound Eye Factors Affecting Sensitivity. Previously, van Hateren et al. (1989) found the dramatic size change in the external facet widths of the dorsal male eye compared to the facet widths in the ventral portion also occurred in the photoreceptors. Specifically, rhabdomere widths in the dorsal region could be in upwards of two and a half times larger than those found in the ventral region (van Hateren et al. 1989). Despite this change, we did not observe a difference in sensitivity as measured by movement at lower light levels between males and females. While diameter is considered one of the most 
important factors affecting sensitivity, the length of the receptor can also play a role (Land and Nilsson 2012). One possible explanation for why the sensitivities are similar is that the male dorsal area, while containing larger diameters, has a shorter ommatidial length. We found, however, that ommatidial lengths were approximately equal in the female compound eye and the dorsal region of male compound eye, and actually longer in the male ventral region.

While in the male ventral portion of the eye the rhabdomeres extend nearly to the basal membrane, the rhabdomeres in the dorsal region taper well before reaching the basal membrane (van Hateren et al. 1989). This in turn makes it difficult to obtain the rhabdomere length in this region. As such, we were unable to measure rhabdomere length in the present study. Future work should investigate rhabdomere length in the male dorsal region as a wider but shorter rhabdomere length may be similar in sensitivity to a narrower but longer rhabdomere.

Other External Factors Affecting Sensitivity. Previous work has indicated that the ocelli can play a role in the onset of activity in response to light changes (Wunderer and De Kramer 1989). That males have larger median and dorsal ocelli than their female counterparts, however, provides support that the lack of difference in onset of activity cannot be attributed to the ocelli.

Future Behavioral Research. Because we confined flies to small spaces, the movement we measured here was walking. Future studies should investigate designs that allow for flight, as we hypothesize that there may be different light thresholds for walking movement versus flying movement. Walking flies have the 
advantage of having tactile stimulation to help their movement and location. In flight, however, flies must largely rely on visual information (Theobald et al. 2007). It may very well be there are two separate light level thresholds, one for walking and the other for flight.

Furthermore, we used an artificial light cycle created by LEDs. While this allowed for complete control over the light cycle values, it may not accurately reflect natural lighting conditions. The LEDs used were poorer in long wavelength light than shorter wavelength light. van Hateren et al. (1989) described the wider dorsal rhabdomeres were capable of higher modes of longer wavelength, so possibly the ability of the dorsal facets to receive light was reduced. Now that the pattern of early movement for larger individuals has been established in a laboratory setting, future work should utilize an experimental design outdoors to incorporate more natural lighting conditions.

Narrowing Down the Functionality of the "Bright Zone". Previously, van Hateren et al. (1989) hypothesized that the "bright zone" was used to either track females in low light levels or to search for them at further distances in higher light levels. The observations from this work seem to support the latter, as C. megacephala males were not more active in lower light levels than females of similar size. These findings suggest that male eye morphology is not used in moving at lower levels of light, as would be required for low light level mate tracking. Although it remains possible that the specialized male eye morphology could be used to track females in low light conditions, without moving earlier to sites with active females they are unlikely to take advantage of it. Future work 
should investigate the possibility that this eye morphology is used to track females at higher levels of luminance at further distances, as also hypothesized by van Hateren et al. (1989).

Overall Conclusions. There are a number of reasons a blow fly would benefit from a larger adult size. For example, larger males are capable of mating with a wider size variety of females (Stoffolano et al. 2000), and can make the females they mate with less receptive to other mates in comparison to smaller males (Cook 1992). Similarly, larger females have been shown to be able to produce more eggs (Wall 1993). Here we have shown another reason why it is beneficial to be a larger blow fly based on visual properties, as larger individuals can move earlier in the morning than their smaller counter parts. Additionally, we have described a new aspect of the behavioral ecology of $C$. megacephala. $C$. megacephala is a fly of forensic importance and it is acknowledged that little is known about the behavior of forensically important blow flies, especially away from a corpse (Tomberlin et al. 2011).

\section{References}

Anderson, G. 2001. Insect succession on carrion and its relationship to determining the time of death, pp. 143-175. In J.H. Byrd and J.L. Castner (eds.) Forensic Entomology: The Utility of Arthropods in Legal Investigations. CRC Press, Boca Raton FL.

Cook, D.F. 1992. The effect of male size on the receptivity in female Lucilia cuprina (Diptera: Calliphoridae). J. Insect Behav. 5: 365-374.

Digby, P.S.B. 1958. Flight activity in the blowfly Calliphora erythrocephala, in relation to light and radiant heat, with special reference to adaptation. J. Exp. Biol. 35: 1-19. 
Emlen, D.J. 1994. Environmental control of horn length dimorphism in the beetle Onthophagous acuminatus (Coleoptera: Scarabaedae). Proc. R. Soc. Lond. B. 256: 131-136.

Emlen, D.J. and H.F. Nijhout. 2000. The development and evolution of exaggerated morphologies in insects. Annu. Rev. Entomol. 45: 661-708.

Emlen, D.J., L.C. Lavine, and E. Ewen-Campen. 2007. On the origin and evolutionary diversification of beetle horns. PNAS. 104: 8661-8668.

Erzinçlioglu, Z. 1996. Naturalists' Handbook 23 Blowflies. The Richmond Publishing Co, Slough, United Kingdom.

Faria, L.D.B., L. Orsi, L.A. Trinca, and W.A.C Godoy. 1999. Larval predation by Chrysomya albiceps on Cochliomyia macellaria, Chrysomya megacephala, and Chrysomya putoria. Entomol. Exp. Appl. 90: 149-155.

Gibert, P., R.B. Huey, and G.W. Gilchrist. 2001. Locomotor performance of Drosophila melanogaster: Interactions among developmental and adult temperatures, age, and geography. Evolution. 55: 205-209.

Hanski, I. 1987. Nutritional ecology of dung- and carrion feeding insects, pp. 837-884. In F. Slansky and J.G Rodriguez (eds.), Nutritional ecology of insects, mites, spiders, and related invertebrates. John Wiley \& Sons, Inc., New York, NY.

Ireland, S., and B. Turner. 2006. The effects of larval crowding and food type on the size and development of the blow fly, Calliphora vomitra. Forensic Sci. Int. 159: 175-181.

Jander, U., and R. Jander. 2002. Allometry and resolution of bee eyes (Apoidea). Arthropod Struct. Dev. 30: 179-193.

Joshi, D.S. 1999. Latitudinal variation in locomotor activity rhythm in adult Drosophila ananassae. Can. J. Zool. 77: 865-870.

Kelber, A., E.J. Warrant, M. Pfaff, R. Wallen, J.C. Theobald, W.T. Wcislo, and R.A. Raguso. 2006. Limit intensity limits foraging activity in nocturnal and crepuscular bees. Behav. Ecol. 17: 63-72.

Koh, K., J.M. Evans, J.C. Hendricks, and A. Sehgal. 2006. A Drosophila model for age-associated changes in sleep:wake cycles. PNAS. 103: 1384313847. 
Kurahashi, H. 1982. Possible origin of a synanthropic fly Chrysomya megacephala in New Guinea (Diptera: Calliphoridae). Monographiae Biologicae. 42: 689-698.

Land, M.F., and H. Eckhart. 1985. Maps of the acute zones of fly eyes. J. Comp. Physiol. A. 156: 525-538.

Land, M.F. 1997. Visual acuity in insects. Annu. Rev. Entomol. 42: 147-177.

Land, M.F., and D-E. Nilsson. 2012. What makes a good eye? In Animal Eyes. Oxford University Press, Inc., New York, NY.

Meyer-Rochow, V.B., and T.F. Lau. 2008. Sexual dimorphism in the compound eye of the moth Operothera brumata (Lepidoptera, Geometridae). Invertebr. Biol. 127: 201-216.

Norris, K.R. 1965. The bionomics of blow flies. Annu. Rev. Entomol. 10: 47-68.

Ribi, W.A., E. Engels, and W. Engels. 1989. Sex and caste specific eye structure in stingless bees and honey bees (Hymenoptera: Trigonidae, Apidae). Entomol. Gener. 14: 233-242.

Shingleton, A.W., W.A. Frankino, T. Flatt, H.F. Nijhout, and D.J. Emlen. 2007. Size and shape: the developmental regulation of static allometry in insects. BioEssays 29: 536-548.

Spaethe, J., and L. Chittka. 2003. Interindividual variation of eye optics and single object resolution in bumblebees. J. Exp. Biol. 206: 3447-3453.

Stavenga, D.G., R. Kruizinga, and H.L Leertouwer. 1990. Dioptrics of the facet lenses of male blow flies Calliphora and Chrysomia. J. Comp. Physiol. A. 166: 365-371.

Stoffolano, J.G., E.Y. Gonzalez, M. Sanchez, J. Kane, K. Velazquez, A.L. Oquendo, G. Sakolsky, P. Schafer, and C.M. Yin. 2000. Relationship between size and mating success in the blow fly Phormia regina (Diptera: Calliphoridae). Ann.Entomol.Soc. Am. 93: 673-677.

Sukontason, Ka., T. Chaiwong, S. Piangjai, S. Upakut, K. Moophayak, and Ko. Sukontason. 2008. Ommatidia of blow fly, house fly, and flesh fly: implication of their vision efficiency. Parasitol. Res. 103: 123-131.

Theobald, J.C., M.M. Coates, W.T. Wcislo, and E.J. Warrant. 2007. Flight performance in night-flying sweat bees suffers at low light levels. J. Exp. Biol. 210: 4034-4042. 
Tomberlin, J.K., R. Mohr, M.E. Benbow, A.M. Tarone, and S. VanLaerhoven. 2011. A roadmap for bridging basic and applied research in forensic entomology. Annu. Rev. Entomol. 56, 401-421.

van Hateren, J.H., R.C. Hardie, A. Rudolph, S.B. Laughlin, and D.G. Stavenga. 1989. The bright zone, a specialized dorsal eye region in the male blow fly Chrysomia megacephala. J. Comp. Physiol. A. 164: 297308.

Wall, R. 1993. The reproductive output of the blowfly Lucilia sericata. J. Insect Physiol. 39: 743-750.

Wells, J.D., and H. Kurahashi. 1994. Chrysomya megacephala development: Rate, variation and the implications for forensic entomology. Jpn. J. Sanit. Zool. 45: 303-309.

Williams, C.R., and M.J. Kokkinn. 2005. Daily patterns of locomotor and sugarfeeding activity of the mosquito Culex annulirostris from geographically isolated populations. Physiol. Entomol. 30: 309-316.

Wunderer, H., and De Kramer, J.J. 1989. Dorsal ocelli and light-induced diurnal activity patterns in the arctiid moth, Creatonotos transiens. J. Insect Physio. 35: 87-95. 
a)

b)

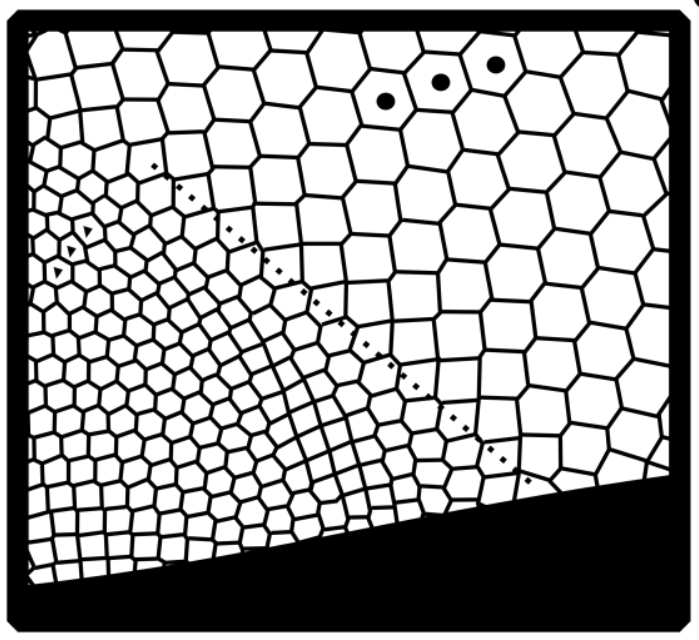

d)

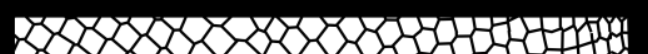

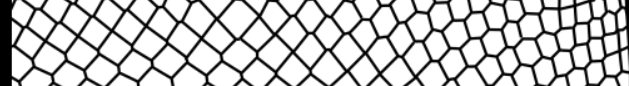
2030

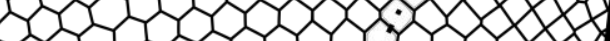
XI)

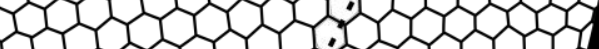

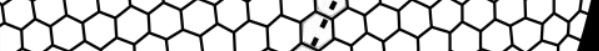

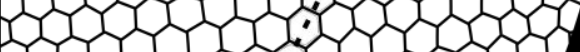

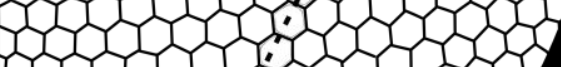

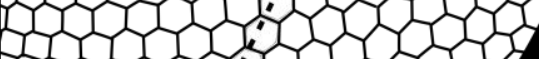
An Dinn 30 12. c)

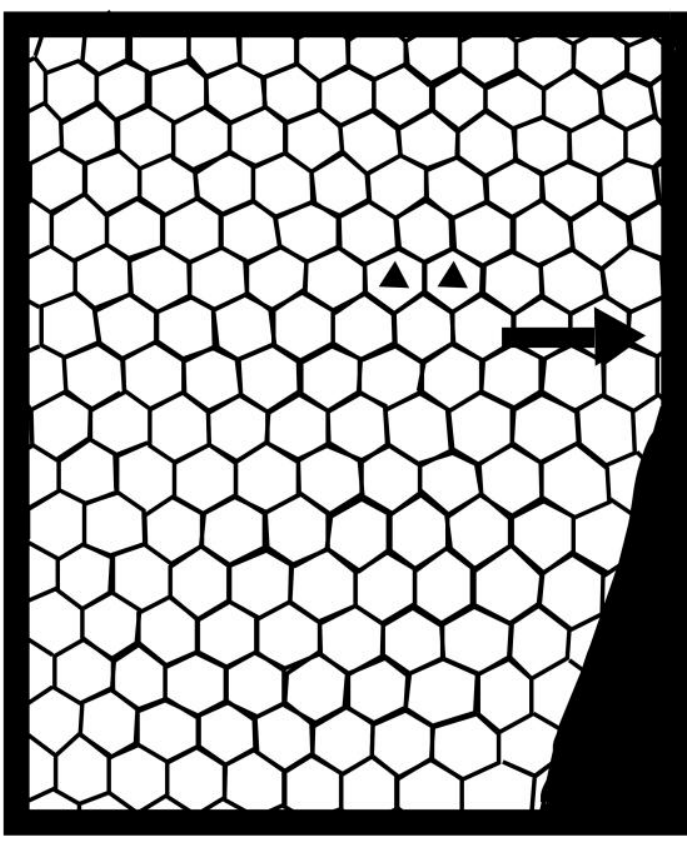


Fig 2.1. The compound eye areas morphological measurements were taken from in Chrysomya megacephala. (a) Photo of male (left) and female (right) heads from C. megacephala. The black dotted circle on the male eye is the area shown in Fig. 2.1b. The black arrow on the female eye points to area shown in Fig. 2.1c. The black lines show the distance measured to determine eye size. (b) Tracing of a male eye replica from the area inside the circle in Fig. 2.1a. The row used to determine the central facet from which the dorsal and ventral ommatidia were measured is marked with a dashed line. The ventral facets measured are indicated by the 3 small triangles. The dorsal facets measured are indicated by the 3 small circles. (c) Tracing of a female eye replica. The two triangles represent the two facets that were measured. The arrow is similar in placement to Fig. 2.1a. (d) Tracing of a male eye replica. Ommatidia were counted to indicate the height of the enlarged area. The dashed line indicates the consecutive rows that were counted. 

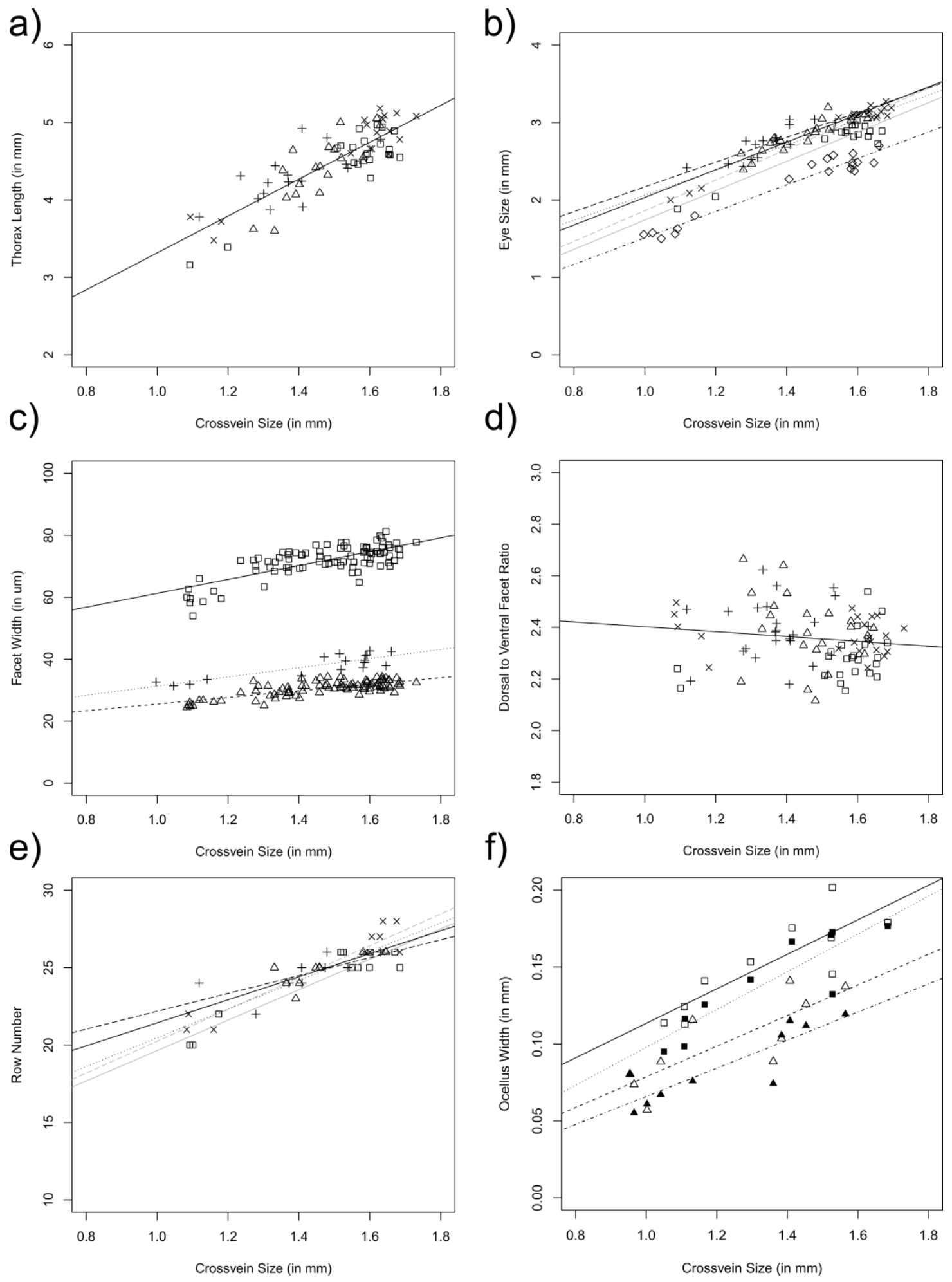
Fig 2.2. The relationship between body size and different eye morphological features. (a) The relationship between thorax length and crossvein length. Each group is represented by a different symbol (males: squares for "FIU colony", $n=21$; triangles for "FIU wild", $n=19$; plus signs for "Keys wild", $n=16$; $x$ 's for "Marathon Female", $n=18$ ). The black regression line is for all groups. (b) The relationship between body size and eye size. Each group is represented by a different symbol (males: squares for "FIU colony", $n=16$; triangles for "FIU wild", $n=20$; plus signs for "Keys wild", $n=18$; x's for "Marathon Female", $n=19$; females: diamonds, $n=19$ ). The dotted black regression line is for the entire group of males. The gray regression lines are for the two groups originating from a single egg clutch. The black regression lines (with the exception of the dotted one) are for the wild caught groups. The solid line indicates an origin on the FIU campus. A long dashed line indicates an origin from the Florida Keys. The dot-dash line is for females. (c) The relationship between body size and facet width. The squares are male dorsal facets $(n=91)$. The triangles are male ventral facets $(n=91)$. The plus signs are female facets $(n=17)$. (d) Dorsal to ventral ratio versus crossvein size for male individuals. Each group is represented by a different symbol (males: squares for "FIU colony", $n=23$; triangles for "FIU wild", $n=22$; plus signs for "Keys wild", $n=24$; $x$ 's for "Marathon Female", $n=22$ ). (e) The relationship between body size and height of the dorsal area in ommatidial rows. Each group is represented by a different symbol (males: squares for "FIU colony", $\mathrm{n}=11$; triangles for "FIU wild", $n=10$; plus signs for "Keys wild", $n=9$; $x$ 's for "Marathon Female", $n=11$ ). The dotted regression line is for the entire group of males. The gray regression lines are for the two groups originating from a single egg clutch. The black regression lines (with the exception of the dotted one) are for the wild caught groups. The solid line indicates an origin on the FIU campus. A dashed line indicates an origin from the Florida Keys. (f) The relationship between body size and ocellus width. The squares are for males, the triangles are for females. Empty shapes correspond to median ocellus, filled in shapes correspond to lateral ocellus. The solid regression line is for the median male ocellus. The dotted line is for lateral male ocellus. The dashed solid regression line is for the median female ocellus. The dot-dash regression line is for the lateral female ocellus. 

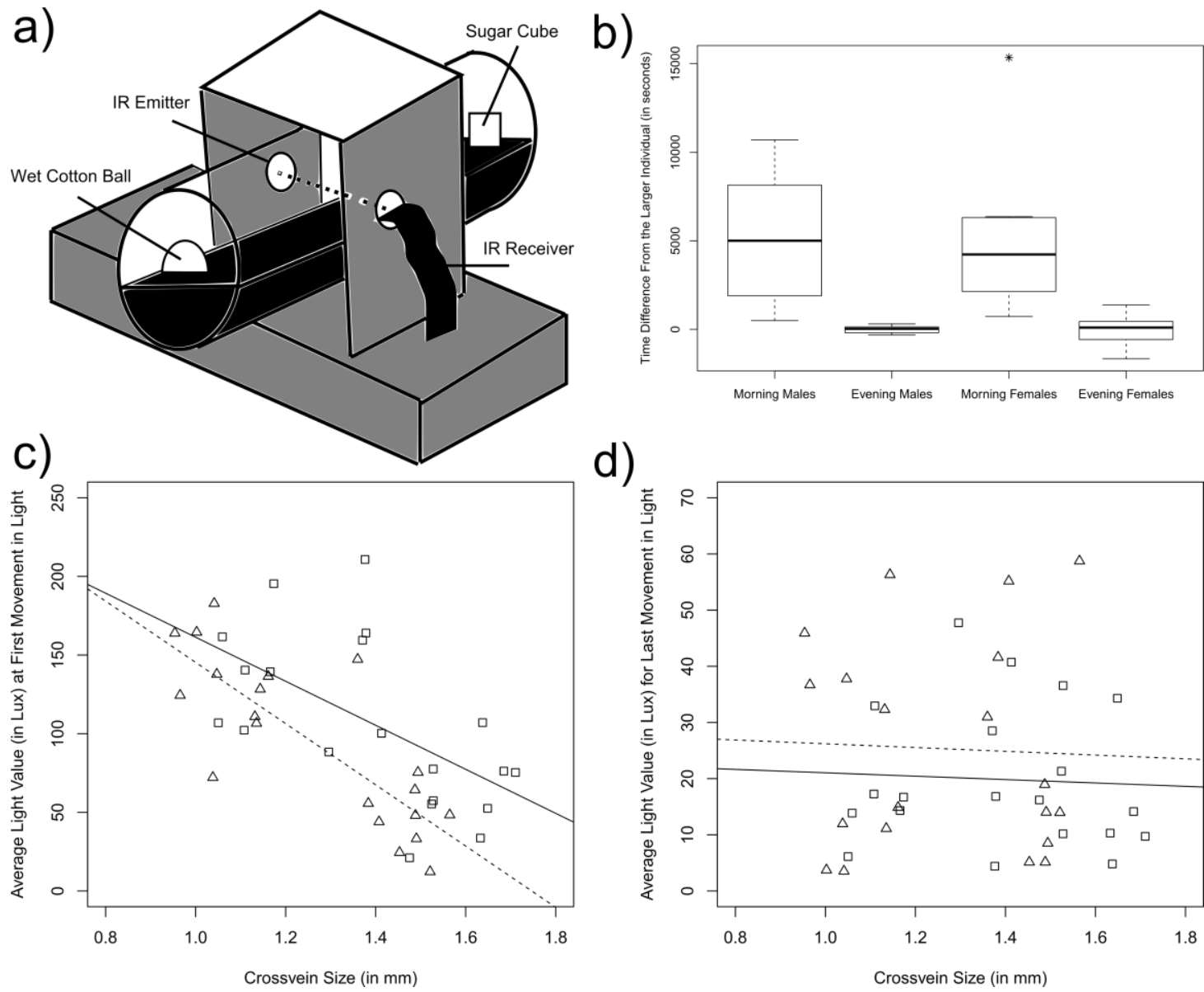

d)

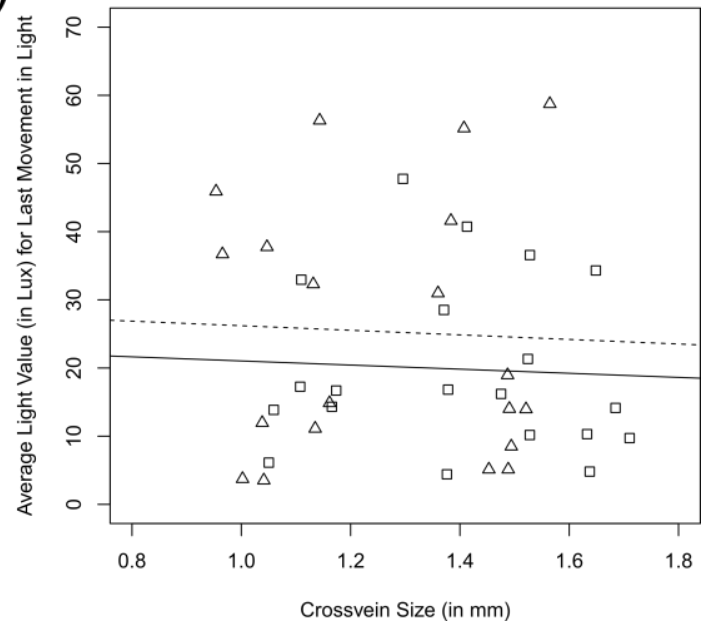

Fig. 2.3. Behavioral analysis of $C$. megacephala daily movement. (a) Drawing of one infrared sensor used. For the final experimental design, two of these were present on the same base to allow for simultaneous measurements from two different flies. (b) Comparison of the time differences between first (in the morning) or last (in the evening) movement between smaller and larger individuals ( $n=10$ pairings, two replicates per pairing). The thick bar represents the median. (c) The average light value at the time of the initial cross in the morning after the lights came on. The squares represent the males $(n=20)$; triangles represent the females $(n=20)$. The solid line is the regression for males. The dashed line is the regression for females. (d) The average light value at the time of the last cross in the evening before the lights turned off. The squares represent the males $(n=20)$; triangles represent the females $(n=20)$. The solid line is the regression for males. The dashed line is the regression for females. 
Table 2.1. Results of regression analysis for various morphological features ( $\pm \mathrm{SE}$ ) when compared to crossvein length.

\begin{tabular}{llllll}
\hline Feature & Group & $\mathrm{R}^{2}$ & Intercept & Slope & P-value \\
\hline Thorax Length & All Males & 0.71 & $0.94(0.27)$ & $2.38(0.18)$ & $<0.001$ \\
Eye Size & Females & 0.95 & $-0.20(0.13)$ & $1.71(0.09)$ & $<0.001$ \\
& All Males & 0.78 & $0.45(0.15)$ & $1.62(0.10)$ & $<0.001$ \\
& FIU Colony & 0.89 & $-0.15(0.28)$ & $1.89(0.18)$ & $<0.001$ \\
& FIU Wild & 0.82 & $0.25(0.29)$ & $1.78(0.20)$ & $<0.001$ \\
& Marathon Female & 0.97 & $0.11(0.13)$ & $1.98(0.08)$ & $<0.001$ \\
Facet Width & Keys Wild & 0.75 & $0.57(0.32)$ & $1.60(0.23)$ & $<0.001$ \\
& Male Dorsal & 0.55 & $38.91(3.22)$ & $22.37(2.17)$ & $<0.001$ \\
D-V Facet Ratio & Male Ventral & 0.55 & $14.84(1.52)$ & $10.65(1.02)$ & $<0.001$ \\
Ommatidia Row No. & Female & 0.75 & $16.48(3.19)$ & $14.82(2.19)$ & $<0.001$ \\
& All Males & 0.019 & $2.50(0.11)$ & $-0.09(0.071)$ & 0.19 \\
& All Males & 0.80 & $11.19(1.09)$ & $9.27(0.74)$ & $<0.001$ \\
& FIU Colony & 0.88 & $9.81(1.78)$ & $9.83(1.20)$ & $<0.001$ \\
Median Ocellus Width & FIU Wild & 0.61 & $14.00(3.11)$ & $7.44(2.12)$ & $<0.001$ \\
Lateral Ocellus Width & Marathon Female & 0.91 & $9.96(1.64)$ & $10.82(1.09)$ & 0.038 \\
& Keys Wild & 0.48 & $16.42(3.20)$ & $5.77(2.26)$ & 0.008 \\
& Males & 0.71 & $0.0013(0.034)$ & $0.11(0.025)$ & 0.002 \\
& Females & 0.66 & $-0.021(0.031)$ & $0.10(0.025)$ & 0.004 \\
& Males & 0.79 & $-0.025(0.030)$ & $0.12(0.022)$ & $<0.001$
\end{tabular}


Table 2.2. Ommatidial length ranges (minimum to maximum) for the compound eyes in a female and a male $C$. megacephala of similar size.

\begin{tabular}{llcc}
\hline Sex & Eye Region & Ommatidial Length Range (um) & Crossvein Length (mm) \\
\hline Female & ----- & $238.29-257.26$ & 1.50 \\
Male & Dorsal & $242.90-252.96$ & 1.48 \\
Male & Ventral & $237.94-265.98$ & 1.48
\end{tabular}


Table 2.3. Results of regression analysis for light level at first movement after lights came on or last movement before lights turned off $( \pm \mathrm{SE})$ compared to crossvein length.

\begin{tabular}{llllll}
\hline Time Light Level Taken & Group & $\mathrm{R}^{2}$ & Intercept & Slope & P-value \\
\hline First Movement in Light & Female & 0.65 & $339.9(42.43)$ & $-194.6(33.11)$ & $<0.001$ \\
& Male & 0.34 & $301.28(65.01)$ & $-139.96(46.10)$ & 0.007 \\
Last Movement in Light & Female & 0.001 & $29.55(26.30)$ & $-3.35(20.53)$ & 0.872 \\
& Male & 0.003 & $24.10(18.95)$ & $-3.02(13.44)$ & 0.825
\end{tabular}




\title{
CHAPTER III: The forensically important blow fly, Chrysomya megacephala (Diptera: Calliphoridae), is more likely to walk than fly to carrion at low light levels
}

\begin{abstract}
One factor that influences estimates of time since death using entomological evidence is whether or not blow flies nocturnally oviposit. Field studies focusing on egg laying have found it occurs on an inconsistent basis. A key but poorly understood factor in nocturnal oviposition is a blow fly's ability to locate carrion under low light levels. It has been speculated that blow flies are more likely to walk than fly to carrion during the night, but this has not been empirically tested. We directly compared guided walking versus flying using infrared sensors under low light levels in laboratory conditions for Chrysomya megacephala (F.) (Diptera: Calliphoridae), a blow fly previously described to be nocturnal. We found C. megacephala is more likely to walk than fly towards carrion under low light levels $(p=0.016)$. We did not, however, find differences between males and females for walking $(p=0.48)$ or flying $(p=0.42)$ despite male C. megacephala possessing eyes better suited for increased light capture. These results demonstrate the need to better understand where blow flies go at night, as bodies found within a fly's walking distance are more likely to be colonized.

\section{Introduction}

The age of an immature carrion insect thought to have developed on a corpse provides an estimate of the minimum time since death [1]. Carrion fly
\end{abstract}


adults are relatively inactive at night [2], and if a death investigator assumes that oviposition or larviposition could not have occurred on the victim at night, this may substantially influence a forensic entomology analysis [3].

The circumstances under which that assumption is justified have been the subject of debate. Certainly oviposition can occur in darkness under laboratory conditions [4] and outdoors when flies are enclosed with carrion [5]. Oviposition has also been observed during some field studies at night, both when flies could walk to carrion [3] and when walking to carrion was prevented [6]. At other field sites, however, it was never observed when allowing for both walking and flying to carrion [4, 7-8], when walking was prevented [5], or when walking was impeded by elevating carrion on a platform [9] or hanging it [10]. One possible reason for this inconsistency is that it is unclear how likely it is an adult female will fly to a corpse at night, or if eggs laid at night are more likely if the oviposition medium is close enough for a female to walk to. Furthermore, some authors have not specified the sex of adult flies that flew to bait at night [11], so the relevance concerning nocturnal oviposition was also not obvious.

Because of this uncertainty, it would be useful to distinguish between nocturnal movement of a carrion fly toward decayed meat by walking compared to by flying and also according to the sex of the individual fly. We attempted to make these distinctions by recording the guided walking and flying behavior of Chrysomya megacephala (F.) (Diptera: Calliphoridae) towards a rotting meat source under a range of very low light levels. C. megacephala is unusual for a muscomorph fly in that males have dorsal compound eye ommatidia and ocelli 
that are much larger than those of females [12-13]. Proportionally larger eyes and ocelli are often found in other insects that are active in low light conditions (reviewed in [14]), and the optical properties of the enlarged dorsal eye facets in male C. megacephala include increased light capture [12]. Furthermore, $C$. megacephala adults have been captured at a meat bait in higher numbers than other carrion insects between the periods of sunset and sunrise, though no information regarding the sex of the individuals was provided [11].

We hypothesized both male and female $C$. megacephala are more likely to walk towards carrion than fly at lower light levels, since walking has the benefit of tactile stimulation. We also hypothesized that male C. megacephala would move more often at lower light levels than their female counterparts, due to their specialized eye and ocellus morphology.

\section{Methods}

\section{Study Groups}

In order to reduce the influence of differing genotype on fly behavior [15], for all trials we used C. megacephala from an inbred laboratory colony originating from wild caught individuals at Florida International University. The colony was maintained for 15 months prior to the beginning of the experiment without the addition of wild flies. In order to reduce age related effects on behavior [16-17], individuals compared in a given experiment were from eggs collected from the colony during a 3-hour interval. Larvae developed at $27^{\circ} \mathrm{C}$ and were provided meat until pupation. Upon emergence, adults were separated by sex within 18 hours and entrained at room temperature (range from $20-22^{\circ} \mathrm{C}$ ) at a low light 
simulated day cycle for at least three days, a time period previously applied prior to experimental manipulation in behavioral assays involving both $C$. megacephala [13] and other dipterans [18-20]. Groups of flies were entrained in separate containers outside the experimental chamber utilizing the same light source and provided water and sugar during this period. The total number of individuals separated was dependent upon the number of flies that emerged. The light cycle was generated by 3 LEDs and a microcontroller (Arduino, Italy). The light cycle consisted of a period of 12 hours of lights off, a two hour period of light increase to a maximum level of approximately 7 lux, followed by an eight hour hold at the maximum level, and finally a two hour decrease back to darkness. A maximum light level of 7 lux was chosen as previously there was not a difference in walking movement between males and females at a minimum light level of approximately 9 lux [13]. We therefore wanted to incorporate lower light levels to determine if the previous lack of difference was due to initial light levels being too high. Additionally, C. megacephala oviposition observations have been made at light levels less than 1 lux [6] so we wanted to ensure we included a slow increase around the lower threshold of light levels.

\section{Experimental Chamber}

After entrainment for each trial, a group of 12 same sex individuals from the same age cohort were separated from the larger group and split into groups of six. In all trials, comparisons were always from the same age cohort. The age of the individuals being compared for a specific trial was always the same, though the age could be different between trials. Individuals were separated by 
sex in order to compare sexual specific behavioral differences. These groups of six were released into separate sides of the experimental chamber (Fig. 3.1) which was illuminated by the previously described light cycle, before the lights turned off. These released individuals spent the night in the chamber. Individuals were not able to move between the two sides due to a piece of white poster board that split the chamber into two equal parts. The chamber was a $24.5 \mathrm{~cm} \mathrm{x}$ $24.5 \mathrm{~cm} \times 24.5 \mathrm{~cm}$ cube. The top, bottom, and side containing the infrared sensors were made of clear Plexiglas. The other three sides were made from mosquito netting. Opposite each entrance opening was a $46 \mathrm{~mm}$ by $26 \mathrm{~mm}$ passageway located either at the bottom of the chamber or $108 \mathrm{~mm}$ above the bottom. Insecta-slip (Bioquip, Rancho Dominguez, CA) was placed around the passageway located $108 \mathrm{~mm}$ above the bottom to prevent access by walking. Two sets of infrared emitters and receivers (Jameco Electronics, Belmont, CA) spanned the opening of each passageway. On the other side of the sensors, each passageway was connected to its own smaller chamber which was a $100 \mathrm{~mL}$ clear plastic cup with a $45 \mathrm{~mm}$ diameter opening. The bottom of the cup was removed and replaced with a mesh opening at the end. Thus, one side of the chamber recorded only flight through the exit hole, while the other side recorded either walking or flying.

\section{Behavioral Observations}

A total of 20 female and 17 male trials were completed. These numbers are uneven because we had a greater number of females than males in our experimental cohorts. The chamber was placed in a room that blocked all outside 
light sources. The temperature was recorded after the introduction of the flies in the cage and again after the trial had finished using a thermometer placed next to the set-up and ranged from $20-22^{\circ} \mathrm{C}$. The walking and flying passageways were blocked until at least 90 minutes before the lights came back on. At this time, approximately 100 grams of ground beef that had been sealed and placed in a $26^{\circ} \mathrm{C}$ incubator for two days and at room temperature for one additional day were placed in the middle of the end with the motion sensors (Fig. 3.1). The choice of three day old beef was consistent with previous work investigating adult movement to carrion in low light conditions [21] and personal experience having greater success attracting adult $C$. megacephala with aged rather than fresh beef. The beef was sealed to prevent odor from escaping until required for a trial. The distance from the passageways and the meat was approximately $45 \mathrm{~cm}$. After placement of the meat, the covering of each passageway was removed, allowing flies to pass through the infrared sensors and into that half of the screened second chamber near the beef. The experiment was stopped when the maximum light level was reached, so each trial lasted at least 3.5 hours. Any cross into either chamber (walking or flying) near the meat before the end of the trial was recorded using a custom python script which recorded the side, number, and time of the cross. The infrared motion sensors were calibrated prior to each trial and a blockage of more than $60 \%$ of the light on either of the two sensors was recorded as a cross.

On 11 occasions during the 37 trials, there was a "cross" that was not caused by blow fly activity. Seven of these "crosses" occurred during times when 
the sensor was blocked, so no blow fly would physically be able to cross. The other 4 occurred during the observation period. Part of the data collected when a cross occurred was the reading from a pin on the Arduino that was not connected to any output (values could range from $0-1023$ ). For all 11 of these crosses, this value ranged from 1-5. We passed both a pen and a tethered dead fly through the sensors over 1000 times each on both the walking and flying side (over 4000 times total) and never obtained a single digit number (0-9) for this value (data not shown). As this was a custom built apparatus, the large number of crosses with inanimate objects was done to determine what values were associated with a real known cross to distinguish real crosses from false crosses that could be considered "noise". The 11 "crosses" where a single digit was recorded for this value were not considered for analysis. In contrast, values consistent with those from the known crosses (40-530) were considered for analysis.

Starting just after the initial placement of the flies and ending after the experiment finished, the light level was recorded every 30 seconds using a photocell (Jameco Electronics, Belmont, CA). The light was incrementally increased every minute so the thirty second time period allowed for a reading during every increment. If a cross occurred within the 30 second period between light level readings, the average light level was determined using the readings directly before and after the cross. To convert the photocell readings into lux values, the light levels were recorded at 10 step increments using a light meter (Gossen Starlite 2, Nurnberg, Germany). These values were plotted against the photocell values associated with the same 10 step increments and piecewise 
interpolation was used to estimate light levels not directly recorded as described in [13].

\section{Statistical Analysis}

For all statistical tests, an alpha value of 0.05 was used as the threshold for significance. A one-tailed Fisher's exact test was used to determine whether the number of trials with walking crosses was significantly greater than the number of trials with flying crosses for the following three groups: all combined (male and female trials together) trials; female trials; and male trials. A one-tailed Fisher's exact test was also used to determine whether the number of trials in which walking occurred for males was significantly larger than the number of trials in which walking crosses occurred for females. Similarly, to determine whether the number of trials in which flying males occurred was significantly larger than the number of trials in which flying females occurred.

In addition, we used a one-tailed paired t-test to determine if the light level at the time of the first walking cross was significantly less than the light level at the time of the first cross for flying. If one type of movement occurred (i.e., walking or flying) but the other did not, we used the maximum light level reached following the two hour light increase that day as the light level for no movement to compute the difference. While the data for the light comparisons did not exhibit a normal distribution, the distribution was unimodal, symmetrical, and had a limited variance allowing us to feel justified in using a paired t-test [22]. 


\section{Results}

Flies were recorded both walking and flying, but crosses on the walking side were significantly more common (Fig. 3.2a; $p=0.016$ ). This relative difference was observed for each sex, although not statistically significant when each sex was compared separately (females: $p=0.064$, males: $p=0.13$ ). While males both walked ( $41.8 \%$ to $35 \%)$ and flew (17.6\% to $10 \%)$ on a larger percentage of trials than females (Fig. 3.2a), this difference was not significantly greater for walking $(p=0.48)$ or flying $(p=0.42)$.

There were very few instances of fly movement recorded during complete darkness (Fig. 3.2b). These consisted of two female and one male walking cross, and two male flying crosses.

Figure 3.2c shows a direct comparison of the light levels that walking and flying movement occurred for each trial. A value of zero indicates no difference in light level between walking and flying, a positive value indicates walking at a lower light level than flying, and a negative value indicates flying at a lower light level than walking. As can be seen in Fig. 3.2c, compared to flying flies generally walked at significantly lower average light levels $(t=1.83, d f=36, p=0.038)$. This trend was the same for females, who also walked at significantly lower light levels than they flew $(t=1.74, d f=19, p=0.049)$. Males, however, did not walk at significantly lower light levels than they flew $(t=0.87, d f=16, p=0.20)$.

\section{Discussion}

It has been speculated that a blow fly is more likely to walk than fly to carrion at low light levels [6], but here we provide the first supporting evidence for 
this opinion. We demonstrated that $C$. megacephala is more likely to walk towards carrion than fly under low light level conditions. While acknowledging flies could have also flown through the "walking" side, we believe they more than likely walked. Both low light and fast optic flow increase photon noise (reducing reliability) in vision [23]. Fast optic flow is induced by flying near the ground [24], so these conditions together make it less likely blow flies flew through the low opening on the "walking" side. That there was a significant difference between the number of trials of walking versus flying overall, but not one for each sex individually, probably reflects the lower sample size for the individual sexes. Previous laboratory work evaluating low light level flight to carrion included a meat source inside the same cage flies were placed in, opening up the possibility that flies captured just randomly came in contact with the meat [21]. By placing the carrion outside of the chamber rather than inside, we were able to demonstrate a more stringent test of guided movement as we required the flies to navigate through an opening to get closer to the beef.

Despite the fact that male $C$. megacephala have both compound eyes and ocelli that appear to be better adapted to low light levels than those of females, in this experiment and in previous work [13] there was no evidence males are more active than females at lower light levels. While previous work limited movement to walking by confining flies to tubes [13], we did not see a difference while allowing for flying that would rely more on visual information in the absence of tactile stimulation. 
On only five occasions was a cross detected with the lights off. While Nanzi et al. [11] characterized C. megacephala as "nocturnal", a characterization of $C$. megacephala engaging in crepuscular activity may be more appropriate. It is possible that a large number of the individuals captured by Nanzi et al. [11] were caught in the periods of dawn and dusk. We found that on the campus of Florida International University on a clear half-moon day, light levels at sunrise and sunset can be over 550 lux. For this reason, information regarding the light level rather than the time of day is better suited for determining the possibility of flight. Flight at low light levels consistent with dawn and dusk have previously been reported for female $C$. megacephala, as Singh and Bharti [6] reported three separate observations of nocturnal oviposition on a medium that could not be accessed by crawling. The reported light levels for these days were between 0.70.8 lux [6]. The lowest light level we observed for female flight in our current work was not much greater at 1.63 lux. We did, however, observe two occurrences of male flights with the lights off. This is surprising as previously it was suggested that blow flies were not capable of oriented flight in darkness [25]. The low number of observations means these results should be taken with some reservations.

The small overall average difference in the light levels between walking and flying (about 1 lux) can probably be attributed to the low light cycle. Since we were targeting low light level movement, the range of light differences was very small with a maximum light difference of 7 lux. This could explain why the differences seen in Fig. 3.2c were so small. Light and dark cycles are known to 
be one of the largest contributors to circadian rhythm [20], so entrainment at the same low light level the experiment was conducted at ensured all flies were set to the same conditions prior to experimentation. Incorporating a similar set-up in an outdoor environment should be considered in the future, as it would allow for a more realistic range of light values.

Another factor to consider is whether or not the circadian rhythm is linked to oviposition behavior. Previous work in another blow fly, Calliphora vicina (Diptera: Calliphoridae), showed that eggs are more likely to be laid during light than in darkness [26]. Future work should not only investigate whether a pattern exists for $C$. megacephala, but also focus on determining a finer range of times. For example, here we focused on the transitional period from dark to light, simulating early mornings. If it is found that flies are more likely to lay eggs in the later stages of the day, then future experiments can focus on observing behavior during these times.

Additionally, we used individuals from the same inbred colony in order to reduce the influence that genetics can have on behavior [15]. Now that this pattern has been established in our inbred population, future work should see if the same differences are present when a natural population more representative of what would be found in a forensic case is used.

One potential issue that may have impacted the results for female $C$. megacephala was whether or not the individuals were gravid. It was highly unlikely that the females used were gravid given the relatively short time after eclosion females could have been used (as little as three days post emergence), 
limited time for mating, and lack of a protein meal for egg development. While there is incentive for both a non-gravid and a gravid female to locate carrion, it is possible that the different motivations for doing so would result in changes in behavior. Previously it has been described that behavior can change in insects when searching for a protein meal in contrast with searching for an oviposition site (reviewed in [27]). Future work should use females known to be gravid to determine how this may influence movement under low light levels.

Future work should also investigate where blow flies go at night, as bodies located in close proximity to these locations would be more susceptible to nocturnal oviposition. Furthermore, we only tested movement across a small distance, meaning these results would only be applicable to cases where $C$. megacephala was in close proximity to carrion. An investigation into distance thresholds for walking movements would further aid in understanding situations where blow flies are more likely to colonize a corpse. Another factor related to distance that might have affected our results is whether $C$. megacephala males were less likely to cross because they were already relatively close to the meat. Male C. megacephala can often be observed around but not on carrion, presumably searching for females. It may be that the short distance used here actually had them at a distance they would generally be at around carrion, making them less likely to move. Future work should increase the distance, which would in turn lower the odor concentration, possibly prompting more movement by the males to carrion to position themselves to find females. 
In conclusion, $C$. megacephala movement increased at low light levels when adults are able to walk to carrion versus when carrion could only be accessed by flying. That there was not a statistically significant difference in movement based on sex, despite male $C$. megacephala having compound eyes and ocelli that appear to be designed for increased light capture, show that more experiments are needed to determine the function of this unique eye design.

\section{References}

[1] Catts, EP, Goff, ML. Forensic Entomology in criminal investigations. Annu Rev Entomol 1992; 37: 253-72.

[2] Erzinçlioglu Z. Naturalists' Handbook 23 Blowflies. 1996. The Richmond Publishing Co, Slough, United Kingdom.

[3] Greenberg B. Nocturnal oviposition behavior of blow flies (Diptera: Calliphoridae). J Med Entomol 1990; 27(5): 807-10.

[4] Amendt J, Zehner R, Reckel F. The nocturnal oviposition behavior of blowflies (Diptera: Calliphoridae) in Central Europe and its forensic implications. Forensic Sci Int 2008; 175: 61-4.

[5] George KA, Archer MS, Toop T. Nocturnal colonization behavior of blowflies (Diptera: Calliphoridae) in southeastern Australia. J Forensic Sci 2013; 58(S1): S112-6.

[6] Singh D, Bharti M. Further observations on the nocturnal oviposition behavior of blow flies (Diptera: Calliphoridae). Forensic Sci Int 2007; 3: 94-7.

[7] Stamper T, Debry RW. The nocturnal oviposition behavior of carrion flies in rural and urban environments: methodological problems and forensic implications. Can Soc of Forensic Sci J 2007; 40(4): 173-82.

[8] Stamper T, Davis P, Debry RW. The nocturnal ovipositing behavior of carrion flies in Cincinnati, Ohio. J Forensic Sci 2009; 54(6): 1450-2. 
[9] Tessmer JW, Meek CL, Wright VL. Circadian patterns of oviposition by necrophilous flies (Diptera: Calliphoridae) in southern Louisiana. Southwest Entomol 1995; 20(4): 439-45.

[10] Barnes KM, Grace KA, Bulling MT. Nocturnal oviposition behavior of forensically important Diptera in central England. J Forensic Sci 2015; doi: 10.1111/1556-4029.12841.

[11] Nanzi WA, Nooraidah H, Jeffery J, Azahari AH, Mohd Noor I, Sadiyah I, Lee $\mathrm{HL}$. Distribution and abundance of diurnal and nocturnal dipterous flies in the Federal Territory, Putrajaya. Trop Biomed 2007; 24: 61-6.

[12] van Hateren JH, Hardie RC, Rudolph A, Laughlin SB, Stavenga DG. The bright zone, a specialized dorsal eye region in the male blow fly Chrysomia megacephala. J Comp Physiol A 1989; 164: 297-308.

[13] Smith JL, Palermo NA, Theobald JC, Wells JD. Body size, rather than male eye allometry, explains Chrysomya megacephala (Diptera: Calliphoridae) activity in low light. J Insect Sci 2015; 15(1): 133.

[14] Warrant EJ. Seeing in the dark: vision and visual behavior in nocturnal bees and wasps. J Exp Biol 2008; 211: 1737-46.

[15] Williams, CR, Kokkinn MJ. Daily patterns of locomotor and sugar-feeding activity of the mosquito Culex annulirostris from geographically isolated populations. Physiol Entomol 2005; 30: 309-316.

[16] Gibert, P, Huey RB, Gilchrist GW. Locomotor performance of Drosophila melanogaster: Interactions among developmental and adult temperatures, age, and geography. Evolution 2001; 55: 205-209.

[17] Koh, K, Evans JM, Hendricks JC, Sehgal A. A Drosophila model for ageassociated changes in sleep:wake cycles. PNAS 2006; 103: 13843-47.

[18] Wheeler DA, Hamblen-Coyle MJ, Dushay MS, and Hall JC. Behavior in lightdark cycles of Drosophila mutants that are arrhythmic, blind, or both. J Biol Rhythms 1993; 8: 67-94.

[19] Rieger D, Fraunholz C, Popp J, Bichler D, Dittmann R, Helfrich-Forster C. The fruit fly Drosophila melanogaster favors dim light and times it activity peaks to early dawn and late dusk. J Biol Rhythms 2007; 22: 387-99.

[20] Yoshii T, Vanin S, Costa R, Helfrich-Forster C. Synergic entrainment of Drosophila's circadian clock by light and temperature. J Biol Rhythms 2009; 24: 452-64. 
[21] Wooldridge J, Scarse L, Wall R. Flight activity of the blowflies, Calliphora vomitoria and Lucilia sericata, in the dark. Forensic Sci Int 2007; 172: 947.

[22] Dowdy S, Wearden S, Chilko D. Statistics for Research. 2004. John Wiley \& Sons, Inc., Hoboken, New Jersey.

[23] Snyder AW. Physics of vision in compound eyes. In Handbook of Sensory Physiology. 1979. Springer, Berlin, pp. 225-313.

[24] Land MF, Nilsson D-E. Animal Eyes. 2012. Oxford University Press, Inc., New York, New York.

[25] Zurawski KN, Benbow E, Miller JR, Merritt RW. Examination of nocturnal blow fly (Diptera: Calliphoridae) oviposition on pig carcasses in midMichigan. J Med Entomol 2009; 46(3): 671-9.

[26] George KA, Archer MS, Toop T. Correlation of molecular expression with diel rhythm of oviposition in Calliphora vicina (Robineau-Desvoidy) (Diptera: Calliphoridae) and implications for forensic entomology. J Forensic Sci 2015; 60: S108-15.

[27] Barton Browne L. Physiologically induced changes in resource-oriented behavior. Annu Rev Entomol 1993; 38: 1-25. 


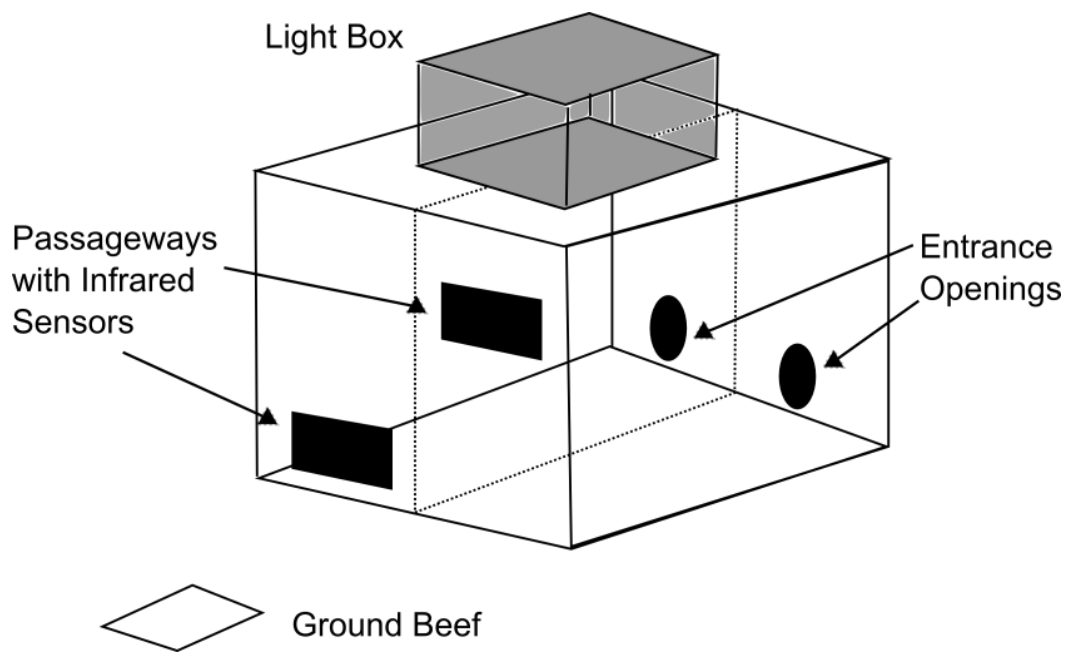

Figure 3.1: Diagram of the experimental chamber. The space within the cube was split into equal halves by an opaque divider (dotted rectangle). Each side had entrance holes (dark ovals) through which flies were introduced before the hole was sealed. A fly in the chamber could move close to, but not touch, a decayed meat bait (ground beef) by entering a screened compartment through a second hole (black squares) that did or did not require flight. The elevated exit hole was surrounded by Insect-a-slip so that no fly could climb that wall of the chamber. An infrared sensor recorded the movement of any fly through the hole to the meat. A box containing three LEDs (gray cube) provided illumination from above. See the text for additional details. 
a)

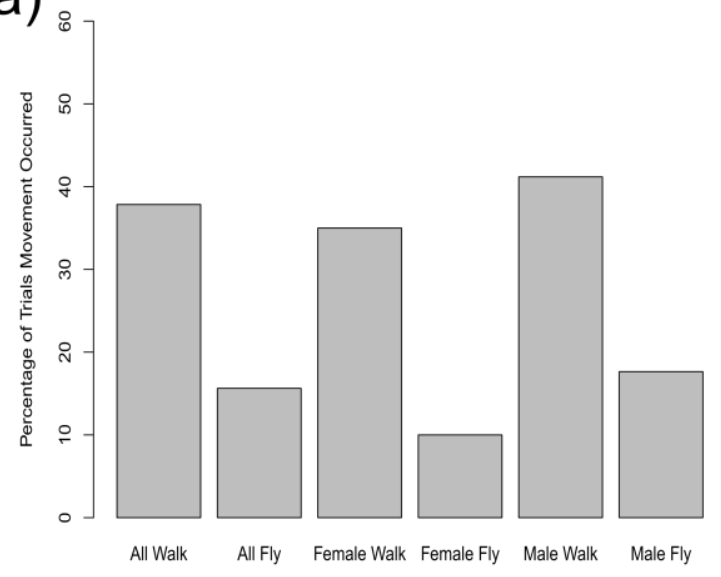

b)

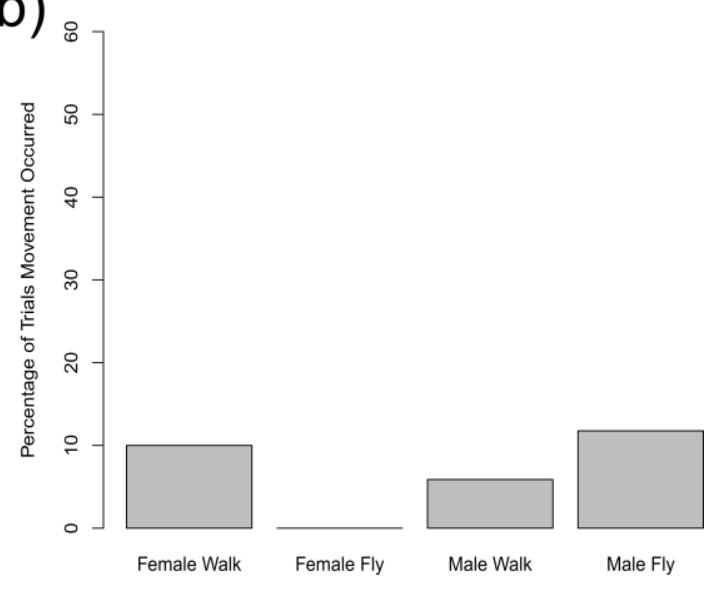

c)

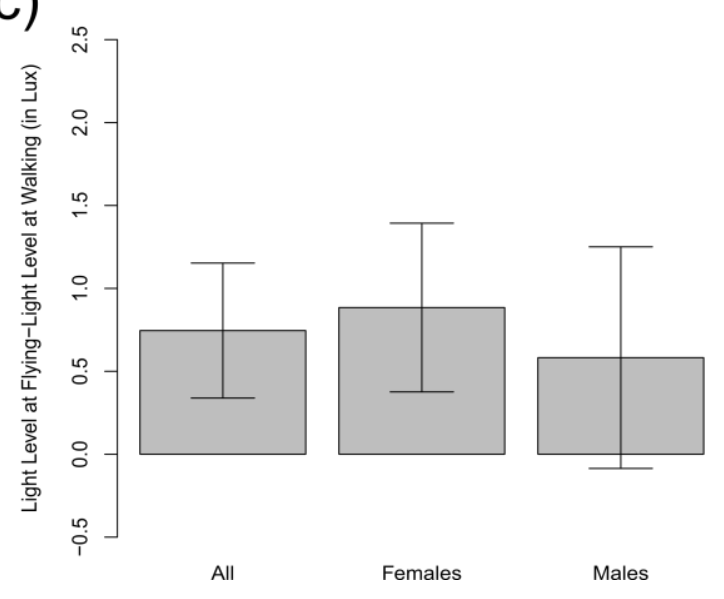

Figure 3.2: Plots of activity patterns during the experiment. (a) The number of trials either walking or flying occurred across all trials $(n=37)$; just trials with females $(n=20)$; and just trials with males $(n=17)$. (b) The number of trials either walking or flying occurred during only the period of darkness ( $n=20$ for females; $\mathrm{n}=17$ for males). (c) The average light level difference (in lux) between walking and flying for males and females. A positive number indicates walking occurred at a lower light level than flying. 


\title{
CHAPTER IV: Isolation of the male-specific transformer exon as a method for immature specimen sex identification in Chrysomya megacephala (Diptera: Calliphoridae)
}

\begin{abstract}
Being able to efficiently differentiate between male and female individuals in the immature forms of insects allows for investigations into sexually dimorphic patterns of growth rates and gene expression. For species lacking sex specific morphological characteristics during these periods, alternative methods must be devised. Commonly, isolation of sex determination genes reveals sex specific band patterns and allow for markers that can be used in insect control. For blow flies, a family that includes flies of medical and forensic importance, sex has previously been identified in some members using the male specific exon in the transformer gene. This gene is relatively conserved between members of the genera Cochliomyia and Lucilia (Diptera: Calliphoridae), and we isolated a portion of this gene in an additional forensically and medically important blow fly genus using the widespread Chrysomya megacephala (F.). We found a relatively high level of conservation between exons 1 and 2 of transformer and were able to amplify a region containing the male specific exon in $C$. megacephala. A sex specific molecular diagnostic test based on the presence of sexually dimorphic PCR product bands showed the expected genotype for adults and intrapuparial period specimens of known sex. The same result could be obtained from single third instar larval specimens, opening up the possibility to not only to determine if
\end{abstract}


development rates are sex dependent, but also to investigate the development of sexually dimorphic traits of interest in C. megacephala.

\section{Introduction}

The ability to identify sex and isolate sex determination genes in the immature forms of blow flies has multiple applications. Some species are livestock pests, leading to programs focused on their sterilization, which utilize sex determination genes as potential markers for control (Scott 2014). Many species of blow flies are forensically important, as the age of an immature specimen found on a corpse is used to estimate the minimum time since death (Catts and Goff 1992) assuming the eggs were laid on the victim after death. This estimate requires a model of larval development rate, which varies as a result of different factors (Wells and LaMotte 2001). Sex, a factor influencing larval development rates in many insects (Teder 2014), has recently been shown to influence development in a blow fly species (Picard et al. 2013). Finally, for an increasing number of blow flies, there are genomes and transcriptomes available (Sze et al. 2012, Wang et al. 2015, Anstead et al. 2015). These tools allow for investigations into genes of interest, many of which likely have sexually dimorphic patterns of gene expression during the larval stages. An issue that hampers the identification of sex in the immature stages, however, is the lack of sexually specific morphological characteristics.

Alternative methods for sex determination in blow flies exist. Karyotyping reveals sex in species for which males have a $Y$ chromosome (Boyes and Shewell 1975). Finding suitable mitotic cells is possible (Azeredo-Espin and 
Pavan 1983, Parise-Maltempi and Avancini 2001), though the efficiency is unclear as insect karyotyping is generally plagued by a limited amount of cells (Popescu and Dutrillaux 2000). Another method is the estimation of genome size that varied between the sexes in some, but not all, of the 10 blow fly species tested (Picard et al. 2012). Finally, a region with one or two male specific exons found in the gene transformer has been used to determine sex in four blow fly species: Lucilia cuprina (Concha and Scott 2009), L. sericata, Cochliomyia hominivorax, and Co. macellaria (Diptera: Calliphoridae) (Li et al. 2013). Transformer is alternatively spliced and only females produce the full length Transformer (TRA) protein that shifts sexual fate toward female (reviewed in Scott et al. 2014). The male specific exon (or exons for L. cuprina, which can have two) is located between the first two exons and contains stop codons, preventing the full length protein in males (reviewed in Scott et al. 2014). The male exon is less conserved than its flanking exons; though portions, including a splice site for TRA and the RNA binding protein Transformer 2 (TRA2), are highly similar between species (reviewed in Scott et al. 2014).

Chrysomya megacephala $(F$.$) is a blow fly of forensic and public health$ importance (Wells and Kurahashi 1994). Genome size does not differ between the sexes (Picard et al. 2012). Males possess a Y chromosome (Parise-Maltempi and Avancini 2001), but we believe that a method to identify sex in the larval stages of $C$. megacephala that could be efficiently performed using only a single specimen would be more useful than doing karyotyping. 
The objective of this work was to identify the male specific exon in the transformer gene of $C$. megacephala and design a molecular method to allow for the identification of sex in the immature stages of this species.

\section{Methods}

Finding Transformer in C. megacephala. C. megacephala genomic DNA from individuals captured in south Florida was extracted, sequenced on the MiSeq (Illumina, San Diego, CA), and assembled into contigs as described in Evenstone (2015). CLC Genomics Workbench (QIAGEN, Valencia, CA) was used to create a local BLAST database from the contigs which was searched against transformer exons 1 and 2 from Co. hominivorax, which flank the male specific exon (Li et al. 2013). These DNA sequences were translated to proteins and conserved amino acid sequences aligned to Co. hominivorax, Co.

macellaria, L. cuprina, and L. sericata. Primers were designed using Primer 3 (Untergrasser et al. 2012) to include the forward primer in exon 1 and the reverse primer in exon 2. The primer sequences used were:

forward: 5'- CCTCATGCTATTGTCCGTGC -3';

reverse: 5'- CGATTTGTTTTGCATTTACCCGT-3'.

RNA Extraction and RT-PCR. Individuals for RNA extraction were either from a C. megacephala colony started in June 2014 from wild individuals and extracted in January 2016 or the direct offspring, second, or fifth generation from a C. megacephala colony originated from wild individuals in March 2016. All founding members were captured in Miami-Dade County, Florida and no wild adults were added once a colony was initiated. RNA was isolated using 
individuals from the following groups: six samples consisting of the heads of two adults (three pairs of males and three pairs of females), six samples consisting of the heads of two intrapuparial period (Martin-Vega et al. 2016) individuals (three pairs of males and three pairs of females; sex known based on our observation that enlarged dorsal ommatidia present in the adult compound eye, Kurahashi 1982, are visible in the later intrapuparial period), 40 whole intrapuparial period individuals from the same egg clutch extracted in 12 hour intervals across 48 hours (sex known for 20); five larval samples of unknown sex reared at $27^{\circ} \mathrm{C}$ for 68.67 hours, and two egg clutches laid within three hours of extraction at room temperature $\left(20-22^{\circ} \mathrm{C}\right)$. For all specimens, individuals were killed and immediately macerated using a sterile plastic pestle. For the adult, intrapuparial period head, and egg specimens, RNA was isolated using the RNeasy Plus Kit (QIAGEN, Valencia, CA) after running the lysate through a QIAShredder column (QIAGEN, Valencia, CA). RNA for these groups was eluted into a final volume of $40 \mathrm{uL}$. For the larval and whole intrapuparial specimens, a single individual was extracted using the Direct-zol RNA Mini-Prep Plus kit (Zymo Research, Irvine, CA) into a final elution volume of $100 \mathrm{uL}$ and $60 \mathrm{uL}$, respectively. All extracts were quantified using a NanoDrop (Thermo Scientific, Wilmington, DE).

A total of 1 ug of RNA was reverse transcribed for each sample using the iScript gDNA Clear cDNA Synthesis Kit (Bio-Rad, Hercules, CA). No-RT controls were included to detect for any possible genomic DNA contamination. The DNase treatment was omitted for the larval samples because a DNase step was included during RNA extraction. The cDNA was amplified with Promega 2x 
Master Mix (Promega, Madison, WI) and the primer sequences previously listed. The thermal cycling parameters were $94^{\circ} \mathrm{C}$ for $5 \mathrm{~min}$; 34 cycles of $94^{\circ} \mathrm{C}$ for $1 \mathrm{~min}$, $52^{\circ} \mathrm{C}$ for $1 \mathrm{~min}$, and $72^{\circ} \mathrm{C}$ for $1.5 \mathrm{~min}$; and a final extension at $72^{\circ} \mathrm{C}$ for $10 \mathrm{~min}$. PCR product was run on a $\sim 1.5 \%$ agarose gel stained with SYBR Safe (Thermo Fisher, Waltham, MA) and visualized using a UV transilluminator.

Sequencing the Male Exon. The PCR product for the three adult males was purified using EXO-SAP IT (Affymetrix, Santa Clara, CA) and sequenced directly using the same PCR primers and separation detection with an ABI3730XL analyzer (Applied Biosystems, Foster City, CA). Sequence data were analyzed using Sequencher (Gene Codes, Ann Arbor, MI).

\section{Results}

Conservation Level among Blow Flies. A comparison between the amino acids in exons 1 and 2 of transformer in C. megacephala and other blow flies showed consistent levels of conservation (Fig. 1). For exons 1 and 2 (Fig. 1A), C. megacephala has a $65.0 \%$ amino acid sequence identity to Co. macellaria, a $64.0 \%$ sequence identity to Co. hominivorax, a $68.0 \%$ sequence identity to $L$. cuprina, and a $67.0 \%$ sequence identity to $L$. sericata.

The length of the male exon in C. megacephala is $305 \mathrm{bp}$ (Fig. A.6). The 3 ' end of this exon includes the highly conserved TRA/TRA2 binding motif previously described for other blow flies (Li et al. 2013) (Fig. 1B).

Male and Female Specific DNA Amplification Patterns. As can be seen in Figs. $2 \mathrm{~A}$ and $2 \mathrm{~B}$, we generated the expected amplification pattern (male exon amplified or not) for adult and intrapuparial period individuals of known sex. 
For the larval specimens where sex was not known, an individual yielded either a male or female pattern (Fig. 2C). For the eggs, only the female specific pattern was observed (Fig. 2D). In some cases we observed a smaller and less bright band in males (Fig. 2E). This band was not always present in males and was never observed in females.

\section{Discussion}

Here we have described for the first time a molecular test to identify sex for C. megacephala. By using a portion of the transformer gene, as has been previously done in other blow flies (Concha and Scott 2009; Li et al. 2013), we were able to isolate the male specific exon in C. megacephala, allowing for sex identification in the larval forms.

Conservation Extends to a New Genus. This molecular test is dependent upon the level of conservation previously observed in other species extending to a new genus. Overall the relatively high level of conservation is not surprising based on previous comparisons between two genera (Li et al. 2013). That combined exons 1 and 2 are more similar to the Lucilia species than the Cochliomyia species, however, is unexpected given the genus Chrysomya is more closely related to Cochliomyia (Singh and Wells 2013). As only a partial region of $C$. megacephala transformer is reported, sequencing the entire gene in the future may show the expected levels of similarity. Furthermore, the length of exon 2 could be confirmed as we used similar sequences obtained in comparisons with Co. hominivorax but did not sequence further in the 3' direction. 
The TRA/TRA2 binding site previously found in the male exon of other blow flies (Li et al., 2013) is highly conserved, but does differ slightly from both Lucilia and Cochliomyia in different areas. This is not surprising as this represents the description of the male exon in a new genus, and previously small differences existed between across genera comparisons (Li et al. 2013).

\section{Immature Sexing Results Similar to Previously Described Blow Fly}

Species. While we were able to obtain either male or female patterns of expression in the larval specimens, we found eggs exhibited only the female band pattern. The eggs used were extracted within three hours of oviposition at room temperature $\left(20-22^{\circ} \mathrm{C}\right)$. Small amounts of male product have been observed after 2-3 hours in $L$ sericata at room temperature (Li et al. 2013), but not until the first instar in L. cuprina at $21^{\circ} \mathrm{C}$ (Concha and Scott 2009). Future work should investigate when the male product can first be seen for $C$. megacephala.

A benefit to identifying sex in the immature stages is the expression of sexually dimorphic genes can be investigated early in development. For example in $C$. megacephala, a defining sexually dimorphic characteristic is eye morphology (Kurahashi 1982). A major event in eye pattern formation and differentiation in dipterans occurs during the third instar, as the morphogenetic furrow moves across the eye-antennal imaginal disc (Ready et al. 1976). Being able to identify sex in the larval stages, means differences between the sexes in genes being expressed during this time period for $C$. megacephala can now be investigated. 
Future Work and Conclusions. Future work should attempt to isolate regions of genomic DNA to serve as molecular markers for sex identification, a more cost effective alternative than RNA isolation and reverse transcription. One target area could be the $\mathrm{Y}$-chromosome, present in C. megacephala (PariseMaltempi and Avancini 2001), as previously a genetic marker for a male specific diagnostic test was designed using a repetitive element found in the $\mathrm{Y}$ chromosome of the Mediterranean fly, Ceratitis capitata (Zhou et al. 2000).

Concha and Scott (2009) and Li et al. (2013) thoroughly investigated the transformer gene in blow flies with an aim of producing male only strains to manage populations in areas where the flies cause myiasis. Here we focused on using this information solely to devise a marker for sex identification for $C$. megacephala in the immature stages, a tool for investigators interested in sex specific development or sexually dimorphic genes. Given the level of conservation observed here, we suspect that a similar sex identification method could be developed for many blow fly species.

References

Anstead, C.A., P.K. Korhonen, N.D. Young, R.S. Hall, A.R. Jex, S.C. Murali, D.S.T Hughes, S.F. Lee, T. Perry, A.J. Stroehlein, B.R.E. Ansell, B. Breugelmans, A. Hofmann, J. Qu, S. Duggan, S.L. Lee, H. Chao, H. Dinh, Y. Han, H.V. Doddapaneni, K.C. Worley, D.M. Muzny, P. Ioannidis, R.M. Waterhouse, E.M. Zdobnov, P.J. James, N.H. Bagnall, A.C. Kotze, R.A. Gibbs, S. Richards, P. Batterham, and R.B. Gasser. 2015. Lucilia cuprina genome unlocks parasitic fly biology to underpin future interventions. Nat. Comm. 6:7344. 
Azeredo-Espin, A.M.L., and C. Pavan. 1983. Karyotypes and possible regions of origin of three species of Calliphoridae (Diptera) recently introduced in Brazil. Rev. Bras. Genet. 4: 619-638.

Boyes, J.W., and G.E. Shewell. 1975. Cytotaxonomy of Calliphoridae (Diptera). Genetica 45: 435-488.

Catts, E.P., and M.L. Goff. 1992. Forensic entomology in criminal investigations. Annu. Rev. Entomol. 37: 253-72.

Concha, C., and M.J. Scott. 2009. Sexual development in Lucilia cuprina (Diptera: Calliphoridae) is controlled by the transformer gene. Genetics 182: 785-798.

Evenstone, L.A. 2015. Employing limited next generation sequence data for the development of genetic loci of phylogenetic and population genetic utility. M.S. thesis, Florida International University, Miami.

Kurahashi, H. 1982. Possible origin of a synanthropic fly Chrysomya megacephala in New Guinea (Diptera: Calliphoridae). Monographiae Biologicae. 42: 689-698.

Li, F., S.P. Vensko, E.J. Belikoff, and M.J. Scott. 2013. Conservation and sexspecific splicing of the transformer gene in the calliphorids Cochliomyia hominivorax, Cochliomyia macellaria, and Lucilia sericata. PLoS One 8: e56303.

Martin-Vega, D., Martin J.R. Hall, and T.J. Simonsen. 2016. Resolving confusion in the use of concepts and terminology in intrapuparial development studies of cyclorrhaphous Diptera. J. Med. Entomol. doi: 10/1093/jme/tjw081.

Parise-Maltempi, P.P., and R.M.P Avancini. 2001. C-banding and FISH in chromosomes of the blow flies Chrysomya megacephala and Chrysomya putoria (Diptera, Calliphoridae). Mem. Inst. Oswaldo Cruz 96: 371-377.

Picard, C.J., J.S Johnston, and A.M. Tarone. 2012. Genome sizes of forensically relevant Diptera. J. Med. Entomol. 49: 192-197.

Picard, C.J., K. Deblois, F. Tovar, J.L. Bradley, J.S. Johnston, and A.M. Tarone. 2013. Increasing precision in development-based postmortem interval estimates: What's sex got to do with it? J. Med. Entomol. 50: 425431. 
Popescu, P., and B. Dutrillaux. 2000. Preparation of chromosome spreads, pp.11-17. In P. Popescu, H. Hayes, and B. Dutrillaux (eds.), Techniques in Animal Cytogenetics. Springer-Verlag, Berlin.

Ready, D.F., T.E. Hanson, and S. Benzer. 1976. Development of the Drosophila retina, a neurocrystalline lattice. Dev. Bio. 53: 217-240.

Scott, M.J., M.L. Pimsler, and A.M. Tarone. 2014. Sex determination mechanisms in the Calliphoridae (Blow Flies). Sex. Dev. 8: 29-37.

Scott, M.J. 2014. Development and evaluation of male-only strains of the Australian sheep blow fly, Lucilia cuprina. BMC Genetics 15: S3.

Singh, B., and J.D. Wells. 2013. Molecular systematics of the Calliphoridae (Diptera: Oestroidea): evidence from one mitochondrial and three nuclear genes. J. Med. Entomol. 50: 15-23.

Sze, H.S., J.P. Dunham, B. Carey, P.L. Chang, F. Li, R.M. Edman, C. Fjeldsted, M.J. Scott, S.V Nuzhdin, and A.M. Tarone. 2012. A de novo transcriptome assembly of Lucilia sericata (Diptera: Calliphoridae) with predicted alternative splices, single nucleotide polymorphisms, and transcript expression estimates. Insect Mol. Bio. 21: 205-221.

Teder, T. 2014. Sexual size dimorphism requires a corresponding sex difference in development time: a meta-analysis in insects. Functional Ecology 28: 479-486.

Untergasser, A., I. Cutcutache, T. Koressaar, J. Ye, B.C. Faircloth, M. Remm, and S.G. Rozen. 2012. Primer3 - new capabilities and interfaces. Nuc. Acids Res. 40: e115.

Wang, X., M. Xiong C. Lei, and F. Zhu. 2015. The developmental transcriptome of synanthropic fly Chrysomya megacephala and insights into olfactory proteins. BMC Genomics 16: 20.

Wells, J.D., and H. Kurahashi. 1994. Chrysomya megacephala development: Rate, variation and the implications for forensic entomology. Jpn. J. Sanit. Zool. 45: 303-309.

Wells, J.D., and L. LaMotte. 2001. Estimating the postmortem interval, pp.263286. In J.H. Byrd and J.L. Castner (eds.), Forensic Entomology: The utility of arthropods in legal investigations. CRC Press, Boca Raton, FL. 
Zhou, Q., P.M. Untalan, and D.S. Haymer. 2000. Repetitive A-T rich DNA sequences from the $Y$ chromosome of the Mediterranean fruit fly, Ceratitis capitata. Genome 43: 434-437. 


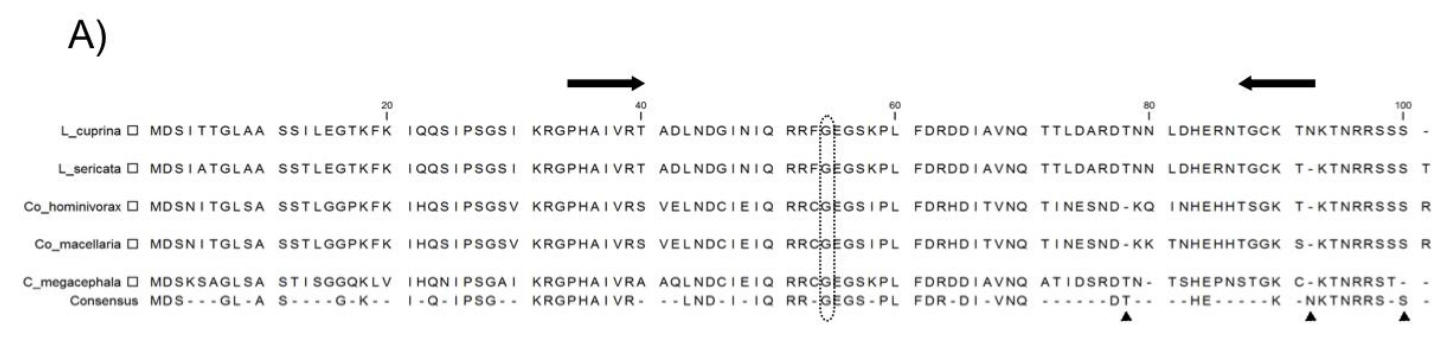

B)

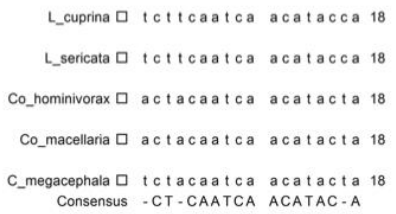

Fig. 4.1. Sequence comparisons for all five blow flies for which a portion of transformer has been described. Sequence data for $L$. cuprina are from Concha and Scott (2009), while sequence data for L. sericata, Co. hominivorax, and Co. macellaria are from Li et al. (2013). A) Comparison of the amino acid sequences for exons 1 and 2. The dotted circle represents the amino acid that is encoded by nucleotides in both exons. The arrows indicate the position of the forward and reverse primers. A triangle underneath the consensus sequence indicates an amino acid that is not present in all species. B) Comparison of the TRA/TRA2 binding site (13 nucleotides long) found in the male specific exon, including the five nucleotide motif adjacent to this region (3' direction). 
A) $\mathrm{MMMFF}$

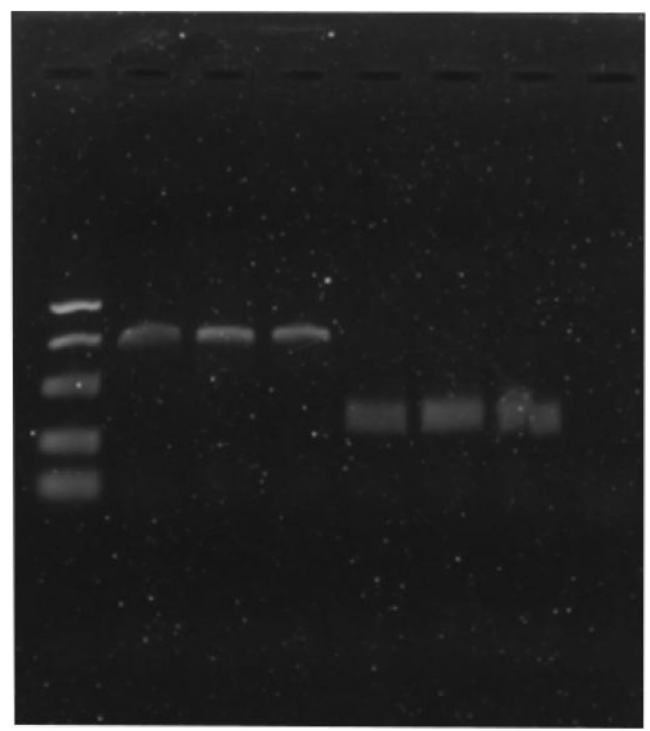

C) $M F F M F$

E)
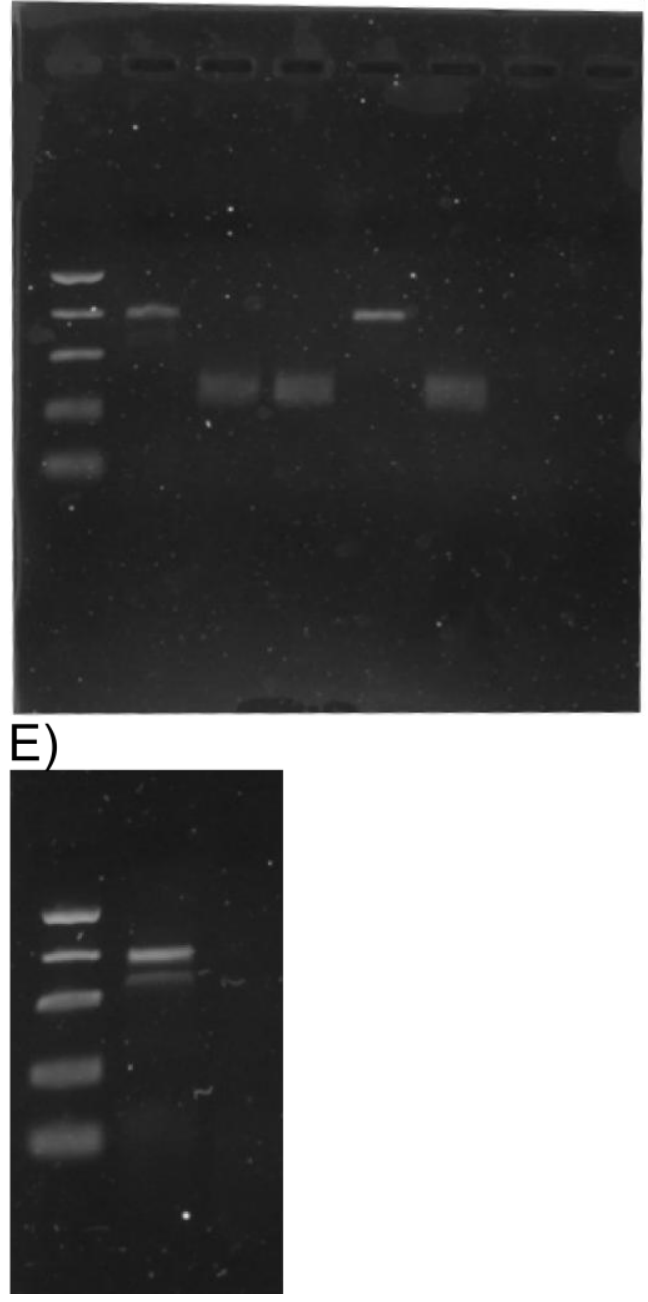

B) $M M M F F$

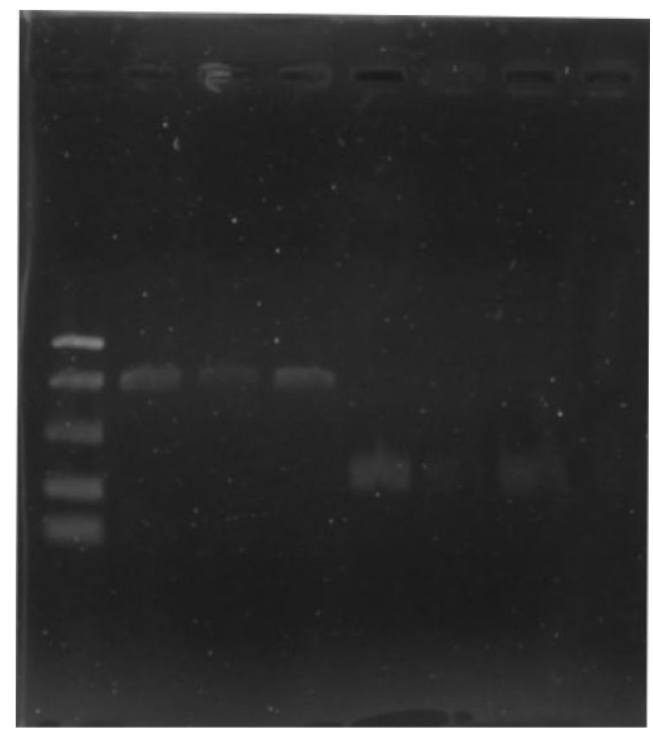

D)

F F

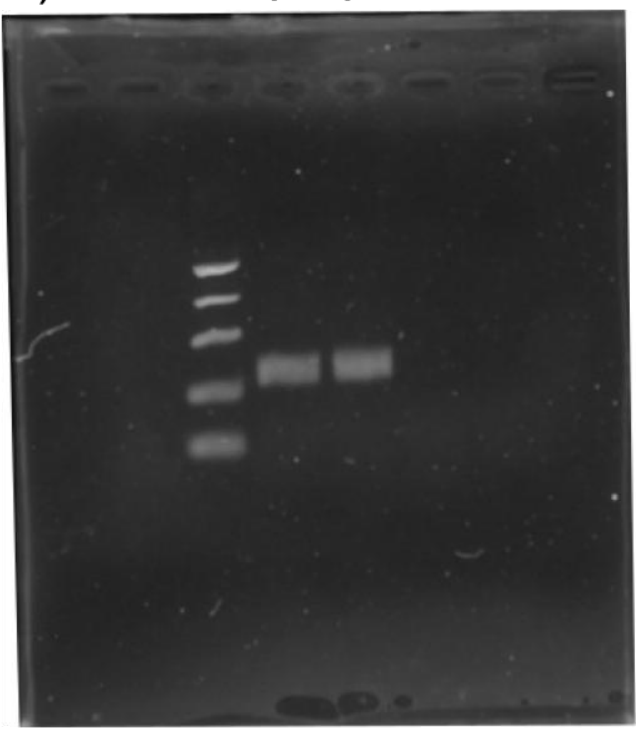


Fig. 4.2. $P C R$ yield gel images showing a male or female band pattern for $(A)$ adults of known sex, (B) intrapuparial period specimens of known sex, (C) unknown larval specimens, and (D) egg clutches. (E) An example of a male intrapuparial period individual showing a second, shorter, and less bright band below the band present in all males. This smaller band is not always present in males, but is never present in females. The ladder used in all images had the following sized fragments (from top to bottom): $766 \mathrm{bp}, 500 \mathrm{bp}, 300 \mathrm{bp}, 150 \mathrm{bp}$, 50bp. A "M" designates male and a "F" designates female specific band patterning. 


\title{
CHAPTER V: Expression of rhodopsin 1 and 3 in Chrysomya megacephala (Diptera: Calliphoridae): a preliminary search for candidate genes responsible for the male eye phenotype
}

\begin{abstract}
Sexually dimorphic differences in compound eye morphology occur in many dipteran species. As much of the same genetic content is shared between males and females, these dimorphic differences can arise as a result of increased expression levels, different locations of expression, or differences in the timing of expression for the same gene. Whereas earlier investigations into sexually specific patterns of gene expression and their influences on sexually dimorphic traits were limited to model organisms, improvements in sequencing technology have given researchers access to genomes and transcriptomes of non-model organisms, allowing similar studies to be conducted in a large number of species. Here, we investigated the pattern of expression in each sex for two genes of interest, rhodopsin $1(r h 1)$ and $3(r h 3)$, which may play a role in the unique compound eye morphology seen in males of the blow fly Chrysomya megacephala (Diptera: Calliphoridae). For $r h 1$, we investigated its temporal pattern of expression during the later stages of intrapuparial development. We found males express rhtearlier in development than females, though the mean differences at these time points were not statistically significant. For $r h 3$, we compared gene expression in adults as previous reports had associated increased rh3 expression with dorsal facet enlargement in other dipterans. In
\end{abstract}


contrast to those reports, we observed higher gene expression in females than males. While this work is preliminary, it provides a basis for future research while representing the first work of comparing sex specific differences in gene expression during the intrapuparial period of blow flies.

\section{Introduction}

Drosophila melanogaster has served as the model organism for dipteran compound eye development. Among the more regular features of the dipteran eye are the eight photoreceptors (Ready et al. 1976). The arrangement of the photoreceptors is relatively uniform, with $\mathrm{R} 1$ through $\mathrm{R} 6$ forming the outer photoreceptors and the more distal R7 located on top of R8 centrally (Tomlinson and Ready 1987a). The function of each of the photoreceptors is linked to the type of rhodopsin gene it expresses. The six outer photoreceptors are used in motion detection and all express rhodopsin 1 (rh1); the two central photoreceptors, R7 and R8, are used for color discrimination and the rhodopsin genes show specific pairwise expression patterns (reviewed in Wernet and Desplan 2004). R7 expresses either $r h 3$ or $r h 4$, paired with $R 8$ expressing either rh5 or rh6, respectively (reviewed in Wernet and Desplan 2004). The sequence in which these rhodopsin genes are expressed is known and is confined to the later portions of the intrapuparial period (Earl and Britt 2006).

Differences in the amount of relative rhodopsin expression from the pattern present in $D$. melanogaster have been linked to morphological changes in the compound eye of other dipterans. For example, an increase in the expression of $r h 3$ among adult members of $D$. mauritiana in comparison with other 
Drosophila species has been linked to enlarged facet size (Posnien et al. 2012). Functional specialization in other dipterans has also been associated with differences in the location of rhodopsin expression. For example, in a region of enlarged facets believed to help track females, male Musca domestica have R7 cells that do not express the typical rhodopsin genes for color discrimination, but instead express the motion detecting $r h 1$ that is normally found in the outer photoreceptors (Hardie 1981).

A fly that exhibits enlarged dorsal facets believed to be involved in mate tracking, and therefore may have patterns of rhodopsin expression different from D. melanogaster, is the male members of the blow fly Chrysomya megacephala (Diptera: Calliphoridae). A defining characteristic of the male C. megacephala compound eye is an area of enlarged dorsal ommatidia, clearly delineated from a region of smaller ventral facets (Kurahashi 1982). The size increase in the dorsal area is not accompanied by a subsequent change in resolution, leading to the area being referred to as the "bright zone" since the function appears to be increased light captured (van Hateren et al. 1989). Additionally, the increase in size seen in the dorsal area extends internally as the photoreceptors are also wider (van Hateren et al. 1989). The functional significance of these changes is hypothesized to either allow males to move in lower light levels or to track females from further distances under higher light levels (van Hateren et al. 1989). Recent work on low light level movement, however, has not shown any differences in movement under low light levels between male and female $C$. megacephala (Smith et al. 2015; Smith et al. 2016). These findings suggest that 
mate tracking is the main reason for the enlarged dorsal facts of the male compound eye.

A common theme among the morphological differences previously discussed involving the enlargement of dorsal facets and the specialization of a dorsal region is both involve genes that are present in individuals with and without the given phenotype. A manner in which more than one phenotype can be controlled by the same genes is a difference in the timing of expression, referred to as transcriptional heterochrony (Skaer et al. 2002). Between different blow fly species, transcriptional heterochrony has been shown to produce gross morphological differences in bristle formation (Skaer et al. 2002).

The C. megacephala male compound eye provides a potential model for understanding how transcriptional heterochrony can lead to gross morphological differences not only between sexes of the same species, but also between closely related species. The closely related sister species of $C$. megacephala, $C$. pacifica, has dorsal facets that are only slightly enlarged (Kurahashi 1991). These two species are genetically similar enough to interbreed and produce offspring with intermediate enlargement of the dorsal facets (J. Wells, personal communication), making transcriptional heterochrony a potential mechanism for the differences observed in compound eye morphology.

While the focus of this work is on the timing and overall expression of genes of interest in relation to compound eye development, it could produce a practical tool as well. C. megacephala is an insect of forensic importance, and a death investigator typically tries to estimate the age of a specimen associated 
with a corpse (Wells and Kurahashi 1994). Knowledge of temporal gene expression could be used to age intrapuparial individuals. Intrapuparial specimens are typically aged by either the development of morphological features (Pujol-Luz and Barros-Cordeiro 2012; Defilippo et al. 2013, Proenca et al. 2014, Brown et al. 2015) or measuring gene expression (Tarone et al. 2011, Boehme et al. 2013, Boehme et al. 2014). Previous gene expression work in blow flies has not considered sex, a factor shown to influence development time for many insect species (Teder 2014), including one species of forensically important blow fly (Picard et al. 2013). For this reason, the investigation into sexually specific patterns of gene expression is also of use to forensic entomologists.

The main objective of this work was to search for sexually dimorphic eye development gene expression in $C$. megacephala to identify genes possibly responsible for the male compound eye phenotype. This investigation employed two approaches. The first approach was to measure $r h 1$ expression according to sex during the later intrapuparial period to determine whether differences in the timing of expression for rh1 play a role in the development of the male compound eye morphology seen in C. megacephala. Previous reports of areas involved in mate tracking have shown rh1 expression in R7 (Hardie et al. 1981), the last photoreceptor to be differentiated. Given the hypothesized role of the $C$. megacephala dorsal facets to be involved in mate detection, we would expect increased $r$ t 1 expression later in males than females concurrent with when the rhodopsin genes typically expressed in $\mathrm{R} 7$ are known to be expressed. 
The second objective was focused on determining if a previous association of increased gene expression observed in enlarged facets held true for male C. megacephala. Specifically the objective was to determine if $r h 3$, previously associated with increased expression levels in adult Drosophila mauritiana known to have enlarged dorsal facets (Posnien et al. 2012), showed a similar pattern with greater expression in adult male $C$. megacephala in comparison with adult females.

\section{Methods}

\section{Designing protocols for measuring expression of $C$. megacephala} rhodopsin 1, rhodopsin 3, and ribosomal protein 49. Utilizing $C$. megacephala genomic information obtained from MiSeq (Illumina, San Diego, CA) sequence data as described in Evenstone (2015), we assembled a local BLAST database of $C$. megacephala contigs and used to it to search for rhodopsin 1 (rh1) sequence data from Calliphora vicina (Huber et al. 1990) with CLC Genomics Workbench (QIAGEN, Valencia, CA). Following alignments between $\mathrm{Ca}$. vicina and $C$. megacephala, we designed primers using Primer 3 (Untergrasser et al. 2012) that amplified partial conserved regions of $r h 1$. In the same manner, we designed primers for $r h 3$ by searching against $\mathrm{Ca}$. vicina sequences (Schmitt et al. 2005) and ribosomal protein 49 (rp49) by searching against known sequences from Lucilia sericata (Tarone and Foran 2011). The partial portion of rp49 was used as a reference gene for this work.

We aligned the C. megacephala partial amino acid sequences of $r h 1$ and rh3 to the same sequences in Ca. vicina (J05596; AF878411) and L. cuprina 
(KNC21061; KNC32561) to determine levels of conservation among blow flies using CLC Genomics Workbench.

RNA Extraction and RT-PCR for Samples During Intrapuparial

Development. We collected eggs from a C. megacephala colony that was initiated from individuals captured in Miami-Dade County and maintained for five generations without the introduction of wild individuals. The eggs used were collected within a two hour time period and based on number and arrangement appeared to be a single clutch (Wells and Kurahashi 1994). Once collected, the eggs were allowed to develop at $27^{\circ} \mathrm{C}$ on chicken liver. After 168 hours at $27^{\circ} \mathrm{C}$, all individuals that had entered the intrapuparial period $(n=118)$ were placed individually into small cups and assigned a number. Eight of these individuals, selected using a random number generator, were extracted after each 12-hour interval from age 192 to 240 hours (Table 5.1). Adult emergence had occurred by age 252 hours. For extraction, the puparium was removed and a brief description of the eye (including eye color and whether or not sex could be identified based on eye morphology; see Table 5.1 for a summary of the composition of individuals extracted at each time point) was recorded. The entire individual was macerated using a sterile plastic pestle in Tri-Reagent. RNA was extracted from these individuals using the Direct-zol Mini Prep Plus Kit (Zymo Research, Irvine, California) and eluted in 60uL. RNA extract quantification was performed using a NanoDrop (Thermo Scientific, Wilmington, DE).

A total of 1 ug of RNA was reverse transcribed as described in Chapter IV. For each set of extractions, reactions containing RNA but without reverse 
transcriptase were included to detect if genomic DNA contamination was present. Sex was determined by eye morphology in older individuals or by the genetic test described in Chapter IV for younger individuals. Following this, we amplified the cDNA for each individual using the $r h 1$ and rp49 primers. Initially, a number of pilot amplifications were performed to establish conditions that represented the exponential phase of amplification (Marone et al. 2003). The primers and associated thermal cycling conditions for each are listed in Table 2. For the intrapuparial samples, $r h 1$ and $r p 49$ were amplified in duplicate.

RNA Extraction and RT-PCR for Adults. We extracted RNA from the heads of adult male and female $C$. megacephala using the RNeasy Mini Kit (QIAGEN, Valencia, CA) following homogenization of the samples with QIAshredder columns (QIAGEN, Valencia, CA). The adults extracted were from a single clutch of eggs that represented the second generation from a colony established from wild individuals captured in Miami Dade County in March 2016. A total of three samples of male heads and three samples of female heads were extracted within 24 hours of adult emergence, with each sample consisting of a two heads each. Flies were decapitated live and macerated using a sterile plastic pestle. RNA extraction was carried out following the manufacturer's instructions with a final elution volume of $40 \mathrm{uL}$.

Reverse transcription to cDNA was carried out as described for the intrapuparial samples. The gene of interest for the adults was $r h 3$ instead of $r h 1$ (Table 2). For the adult samples, $r h 3$ and $r p 49$ were amplified in duplicate. 
Semi-Quantification of Gene Expression. For the samples extracted during the intrapuparial period, amplified product from $r h 1$ and $r p 49$ for each member of an age group was run on the same $1.67 \%$ agarose gel stained with SYBR Safe (Thermo Scientific, Wilmington, DE). For the adult samples, amplified product from $r h 3$ and $r p 49$ for all individuals was run on the same $1.67 \%$ agarose gel.

Gene expression was measured semi-quantitatively by analyzing the gel images in ImageJ (version 1.47; National Institutes of Health; Bethesda, MD, USA). We converted the images to black and white and used the "Gel Analysis" function to quantify the gel bands by measuring the area under the associated peak of the band. For samples in which there was not a single clear peak, the largest discernible peak was used for measurement. All samples were normalized to the 150bp band on the ladder, which was run in every gel, by dividing the value for each band by the value for the 150bp band. Since multiple gels were run to include all samples, this helped control for the variation caused by differences in gels. Each PCR sample for a gene of interest (either $r h 1$ or $r h 3$ ) was loaded directly next to the reference gene. The band intensity for a gene of interest was then normalized to the intensity of its paired reference gene by dividing the intensity of that band by the reference gene (Meadus 2003). The values for background signal noise were not subtracted from gene expression analysis, as these values would not alter the comparison between males and females. 


\section{Statistical Analysis of Gene Expression During the Intrapuparial}

Period and Adult Stages. A one-way ANOVA was performed to determine if the expression of the reference gene, $r p 49$, remained consistent across the five intrapuparial periods measured. For the intrapuparial period, t-tests assuming unequal variance were performed comparing the average amount of $r h 1$ expression at each individual time point between males and females. No correction was applied to control the familywise error rate for conducting multiple t-tests.

For the adults, we compared the average gene expression for $r$ h 3 in adult males and females using a t-test assuming equal variance to determine if a difference existed in the mean expression levels for the two sexes.

\section{Determining Whether Sex-Specific Differences in Development Rate}

Exist. Each individual dissected from the puparium was classified as one of six development stages defined by compound eye pigmentation pattern (Table 5.1), and assigned a number (e.g. $1=$ unpigmented eye, the earliest stage, and $6=$ red eye with grayish body, the final stage). A Mann-Whitney test was used to look for an effect of sex on development stage rank as a function of age.

\section{Results}

High Level of Conservation in the rhodopsin Genes between $C$.

megacephala, Ca. vicina, and L. cuprina. A large number of amino acids from both $r h 1$ (Fig. 5.1A) and rh3 (Fig. 5.1B) are conserved within the Calliphoridae. Considering only the partial region of amino acid shared by all three species for rh1, C. megacephala has an $83.95 \%$ amino acid identity to L. cuprina and an 
$81.48 \%$ amino acid identity to Ca. vicina. For $r h 3$, the level of conservation is even greater with C. megacephala sharing a $95.20 \%$ amino acid identity to $L$. cuprina and a $94.63 \%$ identity to Ca. vicina.

The Expression of rp49 is Relatively Consistent throughout the Later Intrapuparial Period. There was not a significant effect of sample age on mean expression level of $r p 49\left(\mathrm{~F}_{4,35}=0.573, P=0.684\right)$, indicating that $r p 49$ is a suitable reference gene for our purpose.

Expression Pattern of rhodopsin 1 during the Intrapuparial Period.

The overall expression of $r h 1$ increased with time across the intrapuparial period (Fig. 5.2A). While this general trend was expected, what is of more interest here is how it varied according to sex (Fig. 5.2B). Neither males nor females showed any expression for the initial 192 hour period. At both 204 and 216 hours, however, males showed an increase in gene expression with females still showing no appreciable level of expression. At 228 hours, females showed a higher expression level than males; a trend that is reversed at 240 hours when both sexes increased expression. At each of the time points, however, there was no significant difference based on sex (Table 5.3).

Comparison of Adult rhodopsin 3 Expression Pattern in Adult Male and Female C. megacephala. As can be seen in Fig. 5.3, there is a higher level of $r$ 3 expression in females than in males. This difference, however, is not significant $(\mathrm{t}=-2.437, \mathrm{df}=4, P=0.07144)$.

No Sexual Difference in Development Rate Based on Compound Eye Morphology. During the first two time periods, the compound eye was 
unpigmented in all individuals. It was not until 216 hours after oviposition that compound eye color began to appear. The results of the Mann-Whitney test showed no difference in development rate based on sex using eye pigmentation pattern $(U=193.00, P=0.904)$.

\section{Discussion}

High Conservation Levels of rhodopsin Genes in Blow Flies. The high level of conservation in the rhodopsin genes among blow flies is not unexpected, given the previous level of conservation observed between the more distantly related Ca. vicina and D. melanogaster (Huber et al. 1990, Schmitt et al. 2005).

This conservation probably extends to the other rhodopsin genes in $C$. megacephala. The two rhodopsin genes expressed by R8, rh5 and rh6, should be targets of future work in $C$. megacephala male eye morphology as males show only a small R8 photoreceptor in the area of enlarged facets (van Hateren et al. 1989). R8's function is likely solely for cell signaling in this region to differentiate the other photoreceptors, as $\mathrm{R} 8$ is the first photoreceptor to be differentiated (Tomlinson and Ready 1987b). This would make it a potential candidate for sex specific regulation, as one would expect C. megacephala males to have lower levels of $r h 5$ and rh6 expression.

The Expression of $r h 1$ appears to be Earlier in Males. The overall pattern of $r h 1$ being expressed later in intrapuparial period is consistent with previous patterns in D. melanogaster (Kumar and Ready 1995). Of interest here is whether there are differences in the timing of expression between sexes. An early point of interest is the 204 hour time period, where all eight individuals had 
unpigmented compound eyes. The sex ratio at this time period was three males compared to five females, but the only individuals to express $r h 1$ at this time point were two of the three males. While the overall mean difference was not significant, the absence of any unpigmented females expressing $r h 1$ suggests that males may be expressing $r h 1$ earlier. A drawback to this work is the small sample size (discussed in more detail later), but this pattern seems to warrant further investigation.

Additionally, we also saw a spike in male $r h 1$ expression at the 240 hour time point. One working hypothesis was that if males are incorporating $r h 1$ in the central photoreceptor R7, then we would expect that $r h 1$ expression would be increased for a longer period. Further investigation of this time period is warranted.

\section{The Expression of $r h 3$ is Not Associated with Enlarged Facets in}

Adult $\boldsymbol{C}$. megacephala. That the adult male $C$. megacephala did not have an increase in rh3 expression suggests developmental regulation different from the regulation producing dorsally enlarged facets in Drosophila (Posnien et al. 2012). It is worth noting, however, that Posnien et al. (2012) only compared females among different species for $r$ h 3 gene expression. Male $D$. mauritiana also seem to have larger dorsal facets in comparison with males of the other groups used in the study (Posnien et al. 2012), meaning this enlargement may not be influenced by genes that also determine sex. Since we are investigating a difference between sexes of the same species and not a difference between species, we 
would expect a target of genes regulating the male eye morphology in $C$. megacephala to be influenced by sex determination genes.

\section{Determining Whether Absolute Age and Developmental Age are}

Similar Enough for Comparison. Individuals being the same age but at different stages of development are a known issue when looking at temporal gene expression (Earl and Britt 2006, Tarone and Foran 2008). In previous blow fly gene expression work, this issue has been addressed by defining a time of that individuals entered the intrapuparial period and using individuals that have reached that stage within a certain time window. Tarone and Foran (2008) utilized individuals that had entered the intrapuparial period within 24 hour windows of each other and calculated the overall percent of development, while Boehme et al. (2013) used a narrower 17 hour window and made comparisons based on percent of intrapuparial development. Both of these approaches assume the time of the earliest emerging insect as the denominator for calculating percent development and while reducing variation, still allow a time period where it could occur. We only used absolute age and not a measure of percent development. We tried to incorporate morphological information as a way of understanding how development rates could differ. Our methodology also allowed for some variation as can be seen by our description of compound eye morphology at the three later time points. Future studies could benefit by identifying individuals that are the same age and show the same morphological characteristics among more than just a single trait. Also, our specific study would benefit from evaluating development by studying morphology in a trait other than 
the compound eye, as differential patterns of gene expression may be the result of sexually dimorphic development times for the compound eye.

Future work evaluating intrapuparial age for blow flies would benefit by coupling information regarding both morphological characteristics and gene expression. Here, we considered both factors for the first time, though our morphological characteristics were limited to a subjective description of compound eye morphology. Recently, there has been a thorough description of multiple morphological characteristics to determine what combinations of features can be used to assess developmental age (Brown et al. 2015). As more descriptions of this nature become available, charting gene expression with these features will help in determining more precise estimates of intrapuparial age and development. Also, more work involving the objective quantification of development stage is needed. Brown et al. (2015) made strides toward this end by quantifying morphological features with a color chart. In the end, however, color was still grouped by overall discrete color categories, and a way of analysis based solely on quantitative values (ex: RGB values) would provide the most objective method for quantification. Animal color analysis in other fields provides guidelines (Stevens et al. 2007) that could be implemented for use in blow flies using digital cameras, a common element in both research and crime labs.

\section{Utilization of a Gene with Known Expression Patterns for Use in}

Forensics. The relatively close conservation of a number of genes between $D$. melanogaster and blow flies provides a valuable tool as many genes in $D$. melanogaster have characterized expression patterns (Arbeitman et al. 2002). 
Here, we evaluated the expression pattern of a gene that is known to be expressed in the later stages of intrapuparial development and found its expression pattern to be similar to D. melanogaster (Kumar and Ready 1995). As such, rh1 could be used by forensic investigators as a marker for late intrapuparial development. By identifying genes that are known to be expressed at various time points, the creation of a set of candidate genes that are expressed during different intrapuparial stages (ex: early, mid, late) could help investigators interested in determining the age of specimens during this developmental period. Gene expression work with blow fly eggs (Tarone et al. 2007) and late larval/early intrapuparial period specimens (Tarone and Foran 2011) has utilized this approach by targeting genes with known expression patterns. As more genomic and transcriptomic information becomes available for blow flies (Sze et al. 2012, Wang et al. 2015, Anstead et al. 2015), genes targeting specific times in development can be more easily evaluated in multiple species.

\section{Evaluating Whether Differences in rh1 Expression are Sex-Specific}

or Species-Specific. If future work confirms the potential pattern observed here that $r h 1$ has an earlier onset of expression in males than females, next it would be imperative to determine whether this difference is associated with the specific eye morphology found in $C$. megacephala males or if it is just a sex-specific response. A blow fly that does not exhibit the same male eye morphology seen in C. megacephala and for which larval specimen sex can be determined would be 
the ideal candidate. One possibility is $C$. rufifacies, a species in the same genus with females that produce offspring of one sex (Ullerich, 1984).

Limitations of the Current Work. While providing the first comparison of intrapuparial period gene expression between males and females in blow flies, the results of this study are preliminary. One reason this work is preliminary is the small sample size ( $\mathrm{n}=8$ for each sample age), which was also associated with unequal number of each sex for a given sample age. As this was a provisional attempt to identify genes of interest that may be crucial to the development of the derived eye phenotype, we chose to concentrate on 12 hour time periods toward the end of intrapuparial development when rh1 has been shown to be expressed in D. melanogaster (Kumar and Ready 1995). Now that time points of interest have been identified, future work can focus on narrower intervals around these time points.

Another limitation of this work is the use of a semi-quantitative RT-PCR instead of real time quantitative PCR (qPCR). The semi-quantitative approach taken here is an older, less sensitive, and less used technique in comparison to qPCR (vanGuilden et al. 2008). We started with this technique in hopes of finding a time point where expression was present in one sex and not the other, and ultimately did quantify our data to have a general understanding of the relative amount of gene expression levels at each time point. Follow up work utilizing QPCR should be conducted now that general expression patterns have been shown. 


\section{References}

Anstead, C.A., P.K. Korhonen, N.D. Young, R.S. Hall, A.R. Jex, S.C. Murali, D.S.T. Hughes, S.F. Lee, T. Perry, A.J. Stroehlein, B.R.E. Ansell, B. Breugelmans, A. Hofmann, J. Qu, S. Duggan, S.L. Lee, H. Chao, H. Dinh, Y. Han, H.V. Doddapaneni, K.C. Worley, D.M. Muzny, P. loannidis, R.M. Waterhouse, E.M. Zdobnov, P.J. James, N.H. Bagnall, A.C. Kotze, R.A. Gibbs, S. Richards, P. Batterham, and R.B. Gasser. 2015. Lucilia cuprina genome unlocks parasitic fly biology to underpin future interventions. Nat. Comm. 6:7344.

Boehme, P., P. Spahn, J. Amendt, and R. Zehner. 2013. Differential gene expression during metamorphosis: a promising approach for age estimation of forensically important Calliphora vicina pupae (Diptera: Calliphoridae). Int. J. Legal Med. 127: 243-249.

Boehme, P., P. Spahn, J. Amendt, and R. Zehner. 2014. The analysis of temporal gene expression to estimate the age of forensically important blow fly pupae: results from three blind studies. Int. J. Legal Med. 128: 565-573.

Brown, K., A. Thorne, and M. Harvey. 2015. Calliphora vicina (Diptera: Calliphoridae) pupae: a timeline of external morphological development and a new age and PMI estimation tool. Int. J. Legal Med. 129: 835-850/

Cagan, R.L., and D.F. Ready. 1989. The emergence of order in the Drosophila pupal retina. Dev. Bio. 136: 346-362.

Defillipo, F., P. Bounilauri, and M. Dottori. 2013. Effect of temperature on six different developmental landmarks within the pupal stage of the forensically important blow fly Calliphora vicina (Robineau-Desvoidy) (Diptera: Calliphoridae). J. Forensic Sci. doi: 10.1111/1556-4029.12199.

Earl, J.B., and S.G. Britt. 2006. Expression of Drosophila rhodopsins during photoreceptor cell differentiation: insights into R7 and R8 cell subtype commitment. Gene Expr. Patterns 6: 687-694.

Evenstone, L.A. 2015. Employing limited next generation sequence data for the development of genetic loci of phylogenetic and population genetic utility. M.S. thesis, Florida International University, Miami.

Hardie, R.C., N. Franceschini, W. Ribi, and K. Kirschfeld. 1981. Distribution and properties of sex-specific photoreceptors in the fly Musca domestica. J. Comp. Phys. 145: 139-152. 
Huber, A., D.P. Smith, C.S. Zuker, and R. Paulsen. 1990. Opsin of Calliphora peripheral photoreceptors R1-R6. The Journal of Biological Chemistry 265: 17906-17910.

Kumar, J.P., and D.F. Ready. 1995. Rhodopsin plays an essential structural role in Drosophila photoreceptor development. Development 121: 4359-4370.

Kurahashi, H. 1982. Possible origin of a synanthropic fly Chrysomya megacephala in New Guinea (Diptera: Calliphoridae). Monographiae Biologicae. 42: 689-698.

Marone, M., S. Mozetti, D. De Ritis, L. Pierelli, and G. Scambia. 2001. Semiquantitative RT-PCR analysis to assess the expression levels of multiple transcripts from the same sample. Biol. Proceed. Online 3: 19-25.

Meadus, W.J. 2003. A semi-quantitative RT-PCR method to measure the in vivo effect of dietary conjugated linoleic acid on porcine muscle PPAR gene expression. Biol. Proceed. Online 5: 20-28.

Picard, C.J., K. Deblois, F. Tovar, J.L. Bradley, J.S. Johnston, and A.M. Tarone. 2013. Increasing precision in development-based postmortem interval estimates: What's sex got to do with it? J. Med. Entomol. 50: 425431.

Posnien, N., C. Hopfen, M. Hillbrant, M. Ramos-Womack, S. Murat, A. Schonauer, S.L. Herbert, M.D.S. Nunes, S. Arif, C.J. Breuker, C. Schlotterer, P. Mitteroecker, and A.P. McGregor. 2012. Evolution of eye morphology and rhodopsin expression in the Drosophila melanogaster species subgroup. PLOS One 7: e37346.

Proenca, B., A.C. Ribeiro, R.T. Luz, A.M. Aquiar, V.C. Maia, and M.S. Couri. 2014. Intrapuparial development of Chrysomya putoria (Diptera: Calliphoridae). J. Med. Entomol. 51: 908-914.

Pujol-Luz, J.R., and K.B. Barros-Cordeiro. 2012. Intra-puparial development of the females of Chrysomya albiceps (Wiedemann) (Diptera, Calliphoridae). Rev. Bras. Entomol. 56: 269-272.

Ready, D.F., T.E. Hanson, and S. Benzer. 1976. Development of the Drosophila retina, a neurocrystalline lattice. Dev. Bio. 53: 217-240.

Schmitt, A., A. Vogt, K. Friedmann, R. Paulsen, and A. Huber. 2005. Rhodopsin patterning in central photoreceptor cells of the blowfly Calliphora vicina: cloning and characterization of Calliphora rhodopsins Rh3, Rh5, and Rh6. J. Exp. Biol. 208: 1247-1256. 
Smith J.L., N.A. Palermo, J.C. Theobald, and J.D. Wells. 2015. Body size, rather than male eye allometry, explains Chrysomya megacephala (Diptera: Calliphoridae) activity in low light. J. Insect Sci. 15(1): 133.

Smith, J.L., N.A. Palermo, J.C. Theobald, and J.D. Wells. 2016. The forensically important blow fly, Chrysomya megacephala (Diptera: Calliphoridae), is more likely to walk than fly to carrion at low light levels. Forensic Sci. Int. 266: 245-249.

Stevens, M., C.A. Parraga, I.C. Cuthill, J.C. Partridge, and T.S. Troscianko. 2007. Using digital photography to study animal coloration. Biol. J. Linn. Soc. 90: 211-237.

Sze, H.S., J.P. Dunham, B. Carey, P.L. Chang, F. Li, R.M. Edman, C. Fjeldsted, M.J. Scott, S.V Nuzhdin, and A.M. Tarone. 2012. A de novo transcriptome assembly of Lucilia sericata (Diptera: Calliphoridae) with predicted alternative splices, single nucleotide polymorphisms, and transcript expression estimates. Insect Mol. Bio. 21: 205-221.

Tarone, A.M., K.C. Jennings, and D.R. Foran. 2007. Aging blow fly eggs using gene expression: a feasibility study. J. Forensic Sci. 52: 1350-1354.

Tarone, A.M., and D.R. Foran. 2011. Genralized additive models and Lucilia sericata growth: assessing confidence intervals and error rates in forensic entomology. J. Forensic Sci. 53: 942-948.

Tarone, A.M., and D.R. Foran. 2011. Gene expression during blow fly development: improving the precision of age estimates in forensic entomology. J. Forensic Sci. 56: S112-S122.

Teder, T. 2014. Sexual size dimorphism requires a corresponding sex difference in development time: a meta-analysis in insects. Funct. Ecol. 28: 479-486.

Tomlinson, A., and D.F. Ready. 1987a. Cell fate in the Drosophila ommatidium. Dev. Bio. 123: 264-275.

Tomlinson, A., and D.F. Ready. 1987b. Neuronal differentiation in the Drosophila ommatidium. Dev. Bio. 120: 366-376.

Ullerich, F.H. 1984. Analysis of sex determination in the monogenic blow fly Chrysomya rufifacies by pole cell transplantation. Mol. Gen Genet. 193: 479-487. 
Untergasser, A., I. Cutcutache, T. Koressaar, J. Ye, B.C. Faircloth, M.

Remm, and S.G. Rozen. 2012. Primer3 - new capabilities and interfaces.

Nuc. Acids Res. 40: e115.

vanGuilder, H.D., K.E. Vrana, and W.M. Freeman. 2008. Twenty-five years of quantitative PCR for gene expression analysis. BioTechniques 44: 619626.

van Hateren, J.H., R.C. Hardie, A. Rudolph, S.B. Laughlin, and D.G.

Stavenga. 1989. The bright zone, a specialized dorsal eye region in the male blow fly Chrysomia megacephala. J. Comp. Physiol. A. 164: 297308.

Wang, X., M. Xiong C. Lei, and F. Zhu. 2015. The developmental transcriptome of synanthropic fly Chrysomya megacephala and insights into olfactory proteins. BMC Genomics 16: 20.

Wells, J.D., and H. Kurahashi. 1994. Chrysomya megacephala development: Rate, variation and the implications for forensic entomology. Jpn. J. Sanit. Zool. 45: 303-309.

Wernet, M.F., and C. Desplan. 2004. Building a retinal mosaic: cell-fate decision in the fly eye. TRENDS in Cell Biology 14: 576-584. 


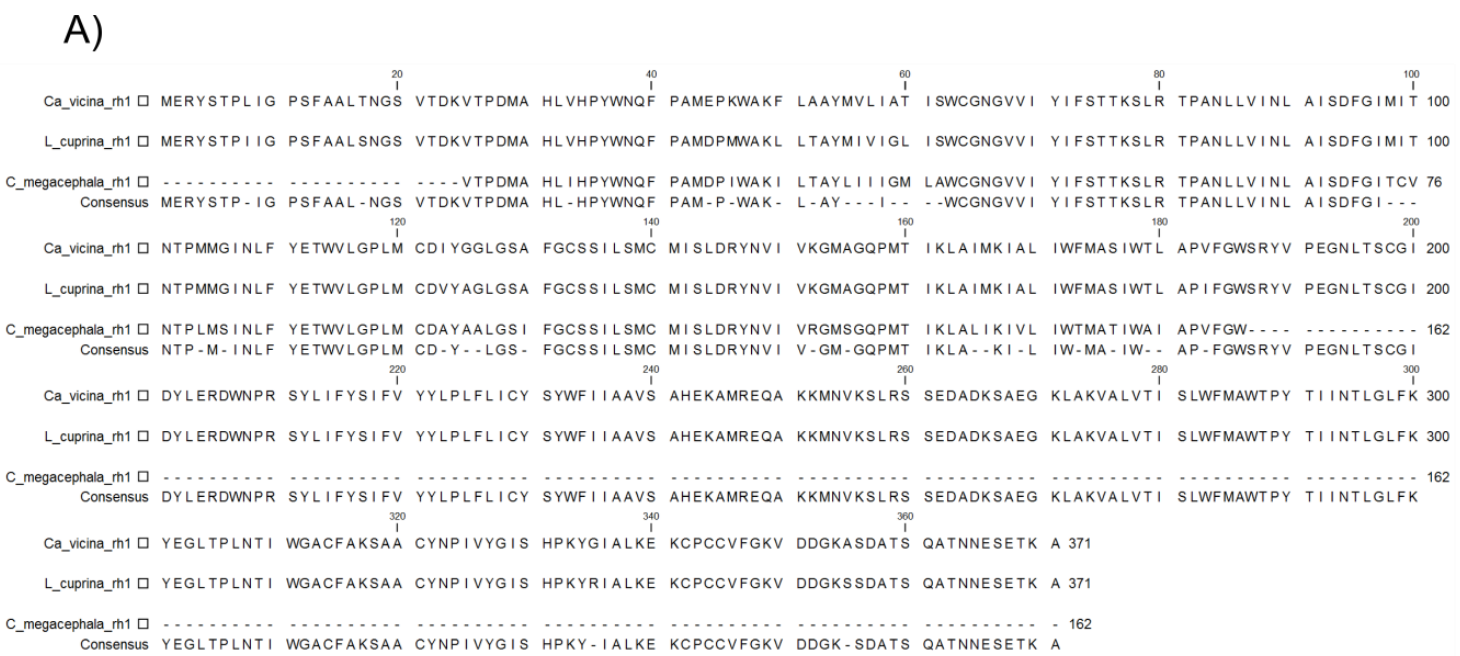

B)

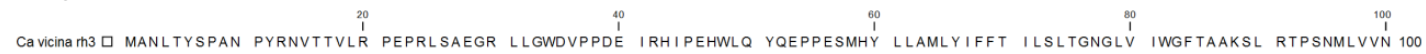
LCuPrinarh3 $\square$ MENVTYSPAN PYRNVSTVLR PDARLSAEGR LLGWDVPPDE IRHIPEHWLQ FQEPPESMHY LLGMLYIFFT ILSITGNGLV IWVFTAAKSL RTPSNILVIN 100

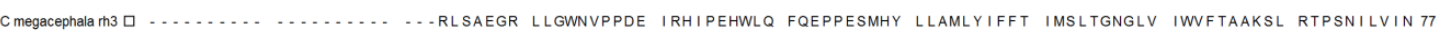
Consensus M-N-TYSPAN PYRNV-TVLR P--RLSAEGR LLGW-VPPDE IRHIPEHWLQ - QEPPESMHY LL-MLYIFFT I-S-TGNGLV IW-FTAAKSL RTPSN-LV-N Cavicinarh3 L LALCDFFMMA KTPIFIYNSF KRGFALGNMG CQIFGIVGSY TGIGASTTNA FIAYDRYNVI TRPLEGKMTH GKAIIMILFI YMYATPFVVA CATESWGRFV 100 LCuprinarh3 L LALCDFFMMA KTPIFIYNSF KRGFALGNLG CQIFAIVGSY TGIGASTTNA FIAYDRYNVI TRPLEGKMTH GKAIIMIIFI YMYATPFVVA CATESWGRFV 200 C megacephalarh3 L LAICDFMMMA KTPIFIYNSF KRGFALGNFG CQIFAIIGSY TGIGAGATNA FIAYDRYNVI TRPLEGKMTH GKAIIMIIFI YMYATPFVVA CATESWGRFV 177 Consensus LA-CDF-MMA KTPIFIYNSF KRGFALGN-G CQIF-I-GSY TGIGA-TNA FIAYDRYNVI TRPLEGKMTH GKAIIMI-FI YMYATPFVVA CATESWGRFV

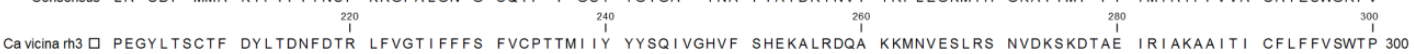
LCuprinarh3 P PEGYLTSCTF DYLTDNFDTR LFVGTIFFFS FVCPTVMIIY YYSQIVGHVF SHEKALREQA KKMNVESLRS NVDKSKDTAE IRIAKAAITI CFLFFVSWTP 300 C megacephalarh3 $\mathrm{P}$ PEGYLTSCTF DYLTDNFDTR LFVGTIFFFS FVCPTCMIVY YYSQIVGHVF SHEKALRDQA KKMNVESLRS NVDKNKDTAE IRIAKAAITI CFLFFVSWTP 277 Consensus PEGYLTSCTF DYLTDNFDTR LFVGTIFFFS FVCPT-MI-Y YYSQIVGHVF SHEKALR-QA KKMNVESLRS NVDK-KDTAE IRIAKAAITI CFLFFVSWTP

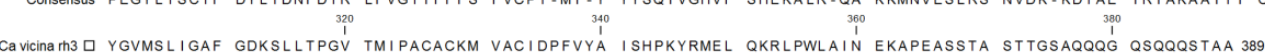
Lcuprinarh3 Y YGVMSLIGAF GDKSLLTPGV TMIPACACKM VACIDPFVYA ISHPKYRLEL QKRLPWLAIN EKAPESTSTV SSSSTAQPPD QGQQQSTAA 389 Cmegacephalarm3 $\square$ YGVMSLIGAF GDKSLLTPGA TMIPACACKL VACIDPFVYA ISHPKYRMEL QKRLPWLAIN EKAPESTSTV SSSSTAQ.............. 356 Consensus YGVMSLIGAF GDKSLLTPG. TMIPACACK - VACIDPFVYA ISHPKYR-EL QKRLPWLAIN EKAPE.-ST - S ..... AQ -... Q-QQQSTA -

Fig. 5.1. Amino acid alignments of rhodopsin genes in three species of blow fly. (A) Alignment of a partial region of $C$. megacephala rhodopsin 1 to Ca. vicina and L. cuprina. (B) Alignment of a partial region of C. megacephala rhodopsin 3 to Ca. vicina and L. cuprina. 

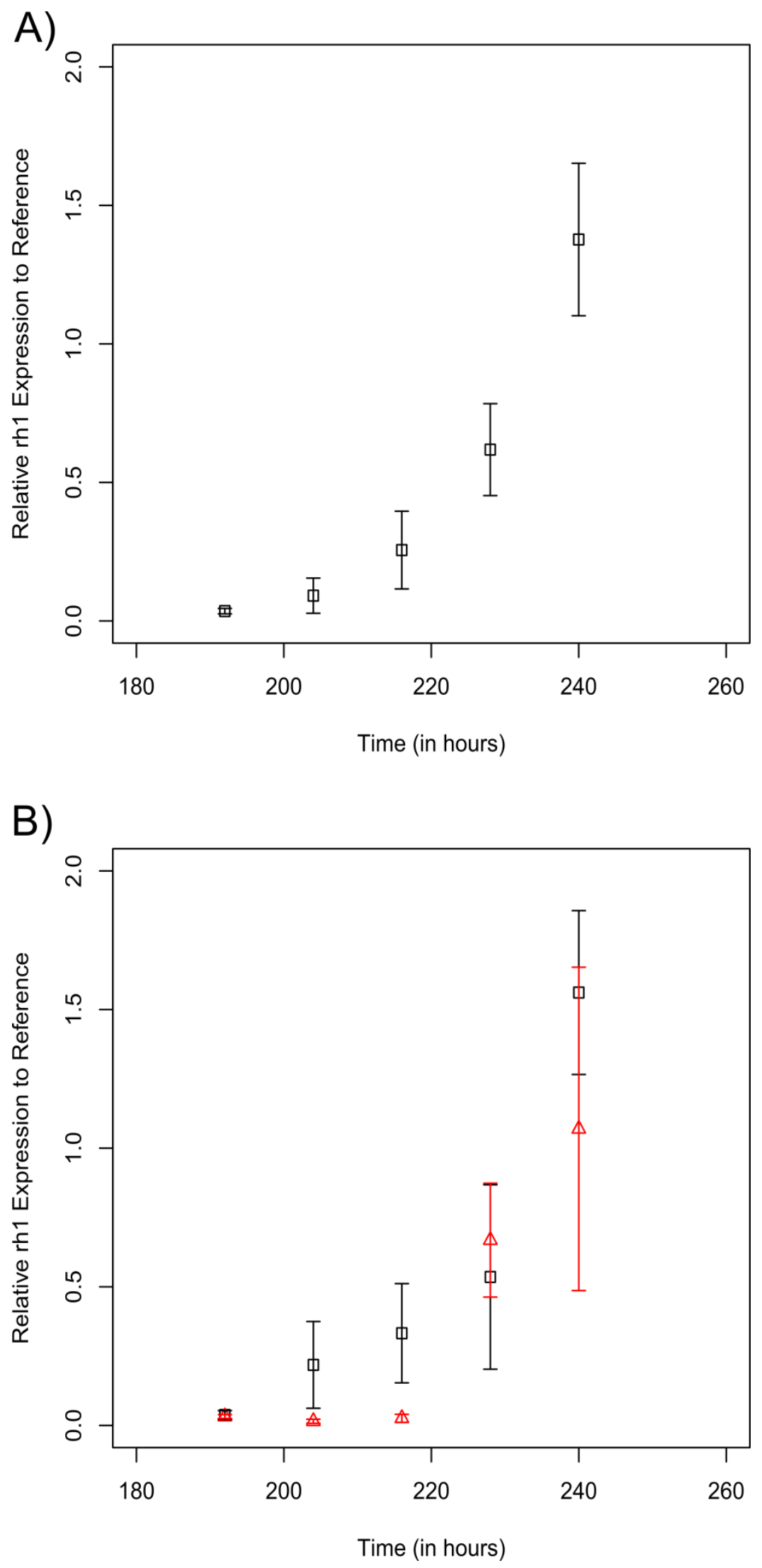
Fig. 5.2. Temporal expression of rhodopsin 1 during the late intrapuparial period. (A) Overall expression pattern of rhodopsin 1 across the five time points. (B) Sexspecific expression of rhodopsin 1 across the five time points. Females are represented by triangles and shown in red. Males are represented by squares. Error bars represent standard error. 


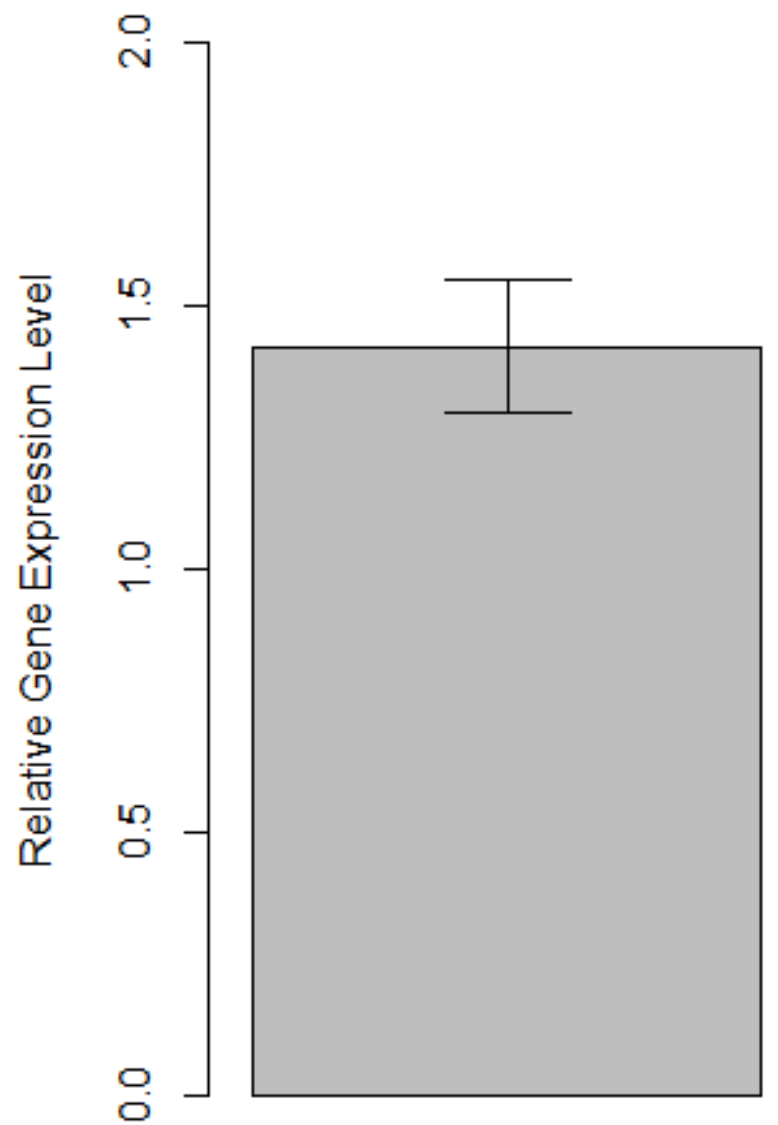

Female Rh3

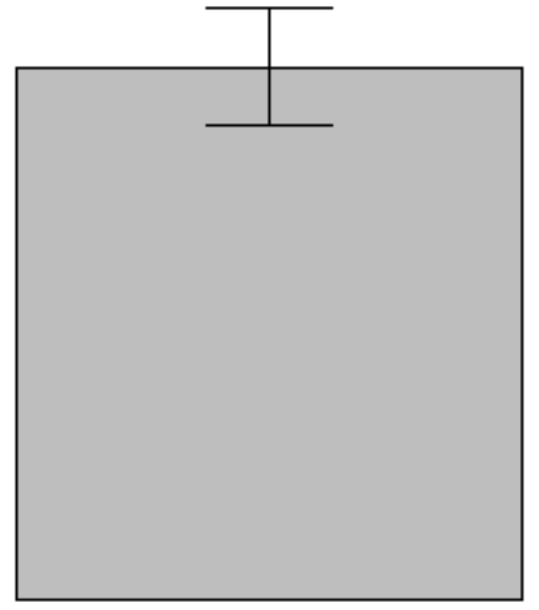

Male Rh3

Fig. 5.3. Plot of the average relative expression level of $r h 3$ in female and male adult $C$. megacephala ( $\mathrm{n}=3$ pairs of heads for each). 
Table 5.1. Summary of the C. megacephala individuals analyzed for each time period, including range of eye colors observed, number of females, number of males, and number of individuals that could have sex determined by eye morphology.

\begin{tabular}{llccc}
\hline Time (hrs) & Eye Colors Observed & Females & Males & Sex ID Eye \\
\hline 192 & Unpigmented & 3 & 5 & 0 \\
204 & Unpigmented & 5 & 3 & 0 \\
216 & Unpigmented, Orange & 2 & 6 & 3 \\
228 & Yellow/Orange, Orange, Red & 5 & 3 & 8 \\
240 & $\begin{array}{l}\text { Orange, Orange/Red, Red } \\
\text { with pigmented body }\end{array}$ & 3 & 5 & 8
\end{tabular}


Table 5.2. Primers and thermal cycling conditions for the amplification of partial portions of the following genes in $C$. megacephala: rhodopsin 1(rh1), rhodopsin 3 (rh3), and ribosomal protein 49 (rp49).

\begin{tabular}{|c|c|c|}
\hline Gene & $\begin{array}{l}\text { Forward Primer }\left(5^{\prime}-3^{\prime}\right) \\
\text { Reverse Primer (5'-3') }\end{array}$ & Thermal Cycling Conditions \\
\hline$\overline{r h 1}$ & $\begin{array}{l}\text { CGATATGGCCCATTTGATTC } \\
\text { GGTGTTCGCAAAGATTTGGT }\end{array}$ & $\begin{array}{l}94^{\circ} \mathrm{C} \text { for } 5 \mathrm{~min} ; 33 \text { cycles of } 94^{\circ} \mathrm{C} \text { for } 1 \mathrm{~min}, 54^{\circ} \mathrm{C} \text { for } 1 \mathrm{~min} \text {, } \\
72^{\circ} \mathrm{C} \text { for } 1 \mathrm{~min} ; 72^{\circ} \mathrm{C} \text { for } 10 \mathrm{~min}\end{array}$ \\
\hline rh3 & $\begin{array}{l}\text { TCTGGAGGGCAAAATGACTC } \\
\text { CAAATCGTCCCCAACTTTCAG }\end{array}$ & $\begin{array}{l}94^{\circ} \mathrm{C} \text { for } 5 \mathrm{~min} ; 33 \text { cycles of } 94^{\circ} \mathrm{C} \text { for } 1 \mathrm{~min}, 53^{\circ} \mathrm{C} \text { for } 1 \mathrm{~min} \text {, } \\
72^{\circ} \mathrm{C} \text { for } 1 \mathrm{~min} ; 72^{\circ} \mathrm{C} \text { for } 10 \mathrm{~min}\end{array}$ \\
\hline rp49 & $\begin{array}{l}\text { CGTCGCTTCAAGGGTCAATAC } \\
\text { CAGTAGACACGGTTTTGCATC }\end{array}$ & $\begin{array}{l}94^{\circ} \mathrm{C} \text { for } 5 \mathrm{~min} ; 23 \text { cycles of } 94^{\circ} \mathrm{C} \text { for } 1 \mathrm{~min}, 58^{\circ} \mathrm{C} \text { for } 1 \mathrm{~min} \text {, } \\
72^{\circ} \mathrm{C} \text { for } 1 \mathrm{~min} ; 72^{\circ} \mathrm{C} \text { for } 10 \mathrm{~min}\end{array}$ \\
\hline
\end{tabular}


Table 5.3. Summary of statistics obtained from t-tests comparing mean $C$. megacephala rh1 expression levels between males and females for each of the five time periods.

\begin{tabular}{llcl}
\hline Time $(\mathrm{hrs})$ & T-value & Degrees of Freedom & P-value \\
\hline 192 & 0.324 & 5.252 & 0.758 \\
204 & 1.298 & 2.009 & 0.323 \\
216 & 1.710 & 5.064 & 0.147 \\
228 & -0.340 & 3.557 & 0.753 \\
240 & 0.752 & 3.058 & 0.506
\end{tabular}




\section{CHAPTER VI. Conclusions}

This dissertation represents a study of the morphology, function, and genetics of the unique compound eye found in male Chrysomya megacephala. In addition to representing insights into a specialized visual system, the role of $C$. megacephala as an insect of forensic importance makes many of these findings relevant to forensic entomologists.

These results say more about what is not the function of the enlarged facets in the dorsal region rather than what is. van Hateren et al. (1989) proposed two possible reasons for this specialized region, including low light level movement. I did not find any difference in low light level movement between male and female C. megacephala when comparing only walking (Smith et al. 2015) and comparing both walking and flying (Smith et al. 2016). Future work should, therefore, focus on testing the other possibility proposed by van Hateren et al. (1989): the enlarged dorsal regions allow for increased contrast sensitivity. Such an outcome has been described in the hoverfly Eristalis tenax, the only other fly for which the "bright zone" has been described (Straw et al. 2006). Electrophysiological work involving experiments testing contrast sensitivity between male and female $C$. megacephala, and between the enlarged dorsal facets and smaller ventral facets in male C. megacephala, would be a logical follow-up to the findings of this dissertation.

The behavior work in this dissertation demonstrated a unique way in which infrared sensors, long utilized for activity studies in flies (ex: Joshi 1999), could 
be used to track movement at low light levels for insects of forensic importance. Previous studies involving forensically important adult insect activity at low light levels have relied on the recording of insect activity with expensive equipment (Barnes et al. 2015) or the capture of insects at traps (Nanzi et al. 2007, Wooldridge et al. 2007). By using custom built infrared sensors, I provide a new way to detect low light level insect movement in an automated and cost effective manner.

A second focus of this work was to investigate the genetic regulation of the male compound eye. Even though the question of how the eye is genetically regulated is not resolved, the answer is much closer based on the results of this dissertation. A major hurdle in the investigation of the genes that are involved in C. megacephala male eye development was the inability to effectively determine sex in the late larval and early intrapuparial stages, when many of the genes are expressed. Taking advantage of recent work in other genera of blow flies (Concha and Scott 2009, Li et al. 2013) and newly acquired genomic information, I was able to isolate a region that could reliably determine sex during these stages. The ability to identify sex in the immature stages opens up numerous possibilities, as now sexually dimorphic features in $C$. megacephala can be investigated. An example is found in this work, where sexually dimorphic expression levels of rhodopsin 1 represents the first study to compare sex specific gene expression in the intrapuparial stage of blow flies. Furthermore, more applied questions such as whether there are differences in development rate based on sex, can now be answered for this species. 
This dissertation has provided information regarding low light level movement, a molecular test for sex determination, and a possible pattern of gene expression involved in the genetic regulation of the male compound eye in $C$. megacephala. These findings have potential applications in both forensic and insect pest management settings. While many questions remain regarding the function and genetic regulation of the male compound eye in C. megacephala, this dissertation has provided tools that will aid future research efforts. As more genomic information becomes available for non-model organisms, questions such as what causes the morphological differences which led to the speciation of closely related species is now possible. The close relationship between $C$. megacephala and C. pacifica, which are considered different species in part because of the male eye morphology, provides a model for understanding how speciation may occur and should be the aim of future research. Similarly, how the sexually dimorphic male compound eye develops differently than that of the female compound eye is closer to being understood than before this dissertation.

\section{References}

Barnes K.M., K.A. Grace, and M.T. Bulling. 2015. Nocturnal oviposition behavior of forensically important Diptera in central England. J. Forensic Sci. doi: 10.1111/1556-4029.12841.

Concha, C., and M.J. Scott. 2009. Sexual development in Lucilia cuprina (Diptera: Calliphoridae) is controlled by the transformer gene. Genetics 182: 785-798.

Joshi, D.S. 1999. Latitudinal variation in locomotor activity rhythm in adult Drosophila ananassae. Can. J. Zool. 77: 865-870. 
Li, F., S.P. Vensko, E.J. Belikoff, and M.J. Scott. 2013. Conservation and sexspecific splicing of the transformer gene in the calliphorids Cochliomyia hominivorax, Cochliomyia macellaria, and Lucilia sericata. PLoS One 8: e56303.

Nanzi W.A., H. Nooraidah, J. Jeffery, A.H. Azahari, I. Mohd Noor, I. Sadiyah, and H.L. Lee. 2007. Distribution and abundance of diurnal and nocturnal dipterous flies in the Federal Territory, Putrajaya. Trop. Biomed. 24: 61-6.

Smith J.L., N.A. Palermo, J.C. Theobald, and J.D. Wells. 2015. Body size, rather than male eye allometry, explains Chrysomya megacephala (Diptera: Calliphoridae) activity in low light. J. Insect Sci. 15(1): 133.

Smith, J.L., N.A. Palermo, J.C. Theobald, and J.D. Wells. 2016. The forensically important blow fly, Chrysomya megacephala (Diptera: Calliphoridae), is more likely to walk than fly to carrion at low light levels. Forensic Sci. Int. 266: 245-249.

Straw, A.D., E.J. Warrant, and D.C. O'Carroll. 2006. A 'bright zone' in male hoverfly (Eristalis tenax) eyes and associated faster motion detection and increased contrast sensitivity. J. Exp. Biol. 209: 4339-4354.

van Hateren, J.H., R.C. Hardie, A. Rudolph, S.B. Laughlin, and D.G. Stavenga. 1989. The bright zone, a specialized dorsal eye region in the male blow fly Chrysomia megacephala. J. Comp. Physiol. A. 164: 297308.

Wooldridge J, L. Scarse, and R. Wall. 2007. Flight activity of the blowflies, Calliphora vomitoria and Lucilia sericata, in the dark. Forensic Sci. Int. 172: 94-7. 


\section{Appendices}

a)

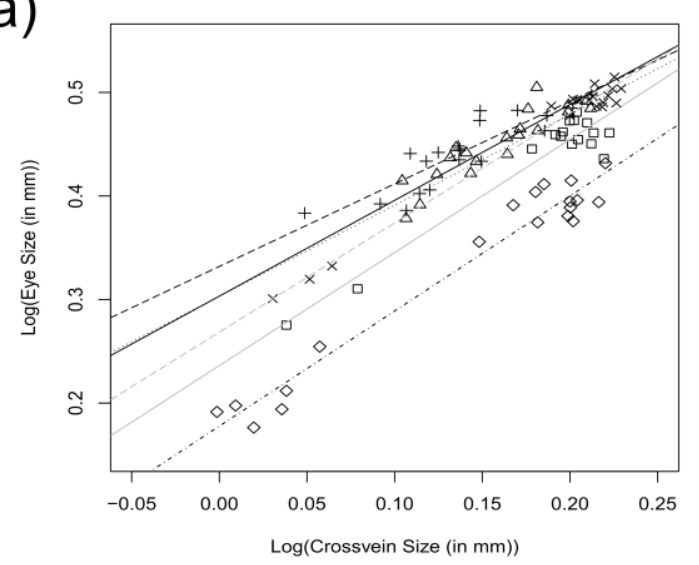

c)

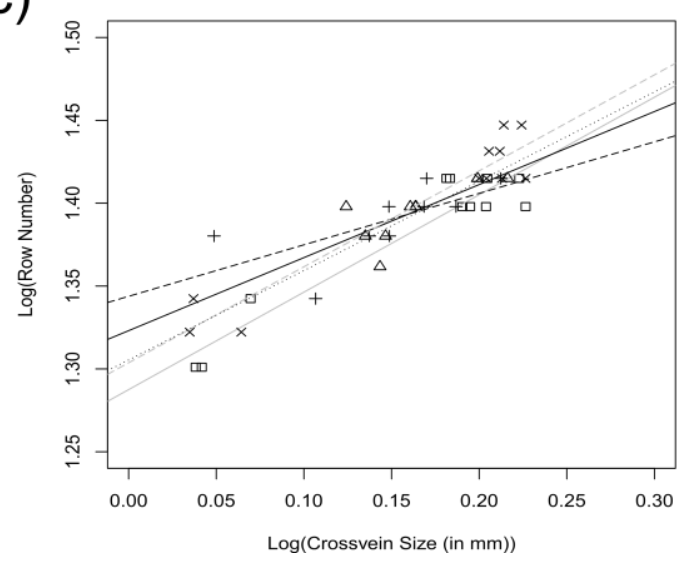

b)

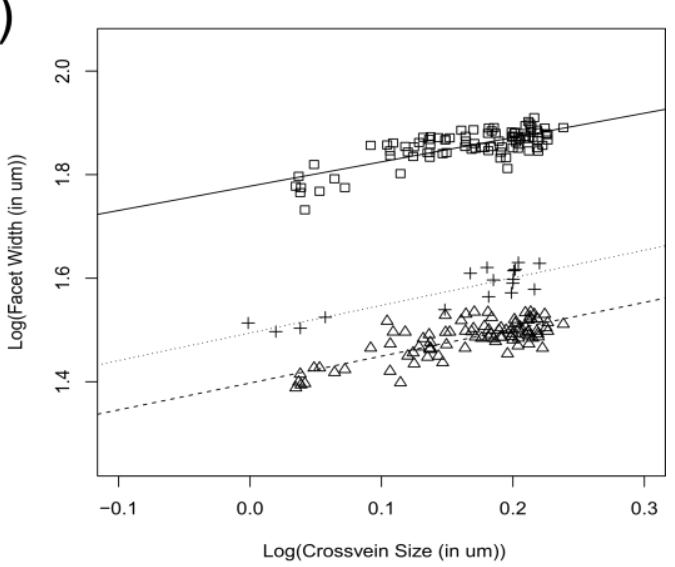

Fig. A.1. Plots of the logarithmic transformation of various eye features in relation to body size (as described in Chapter II). The slopes of these lines show the rate of growth relative to body size. (a) Logarithmic transformation of eye size in comparison to body size. (b) Logarithmic transformation of facet size in comparison to body size. (c) Logarithmic transformation of height of the dorsal area (in ommatidia) in comparison to body size. 
a)

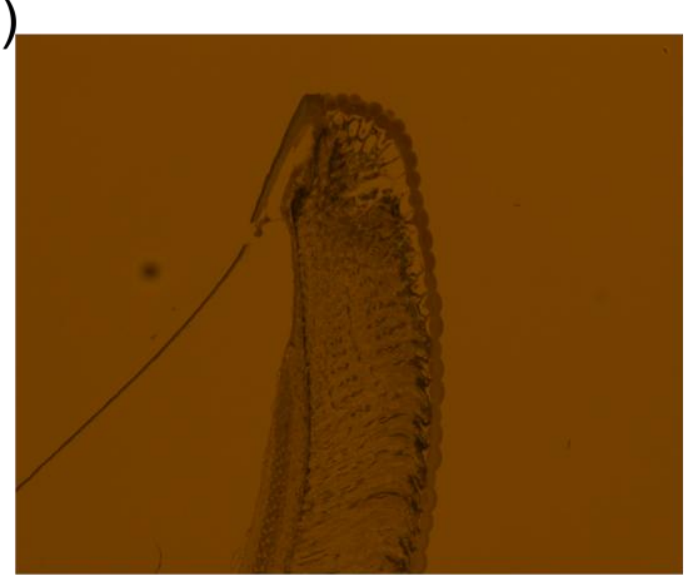

b)

c)

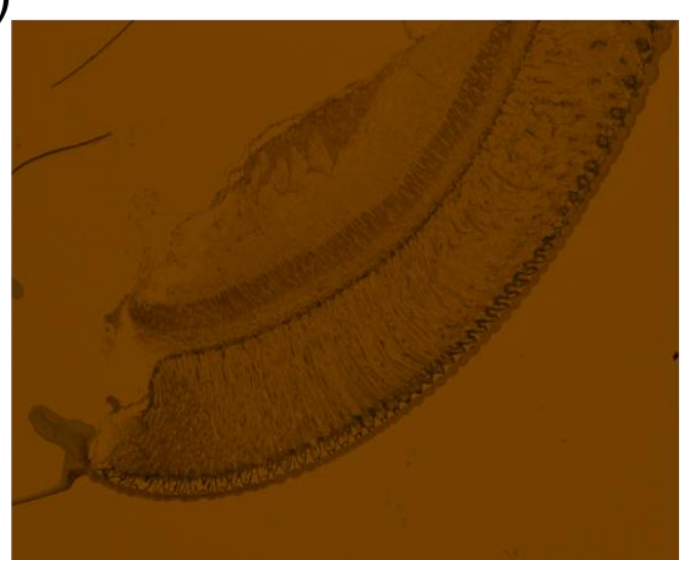

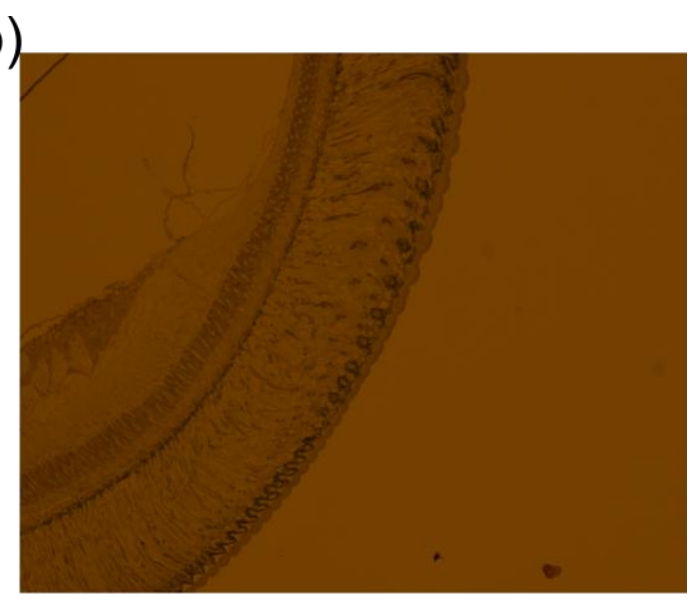

Fig. A.2. Male compound eye histology photos (100x). Each photo is of the same cross section. (a) Section through the dorsal portion of the eye. (b) Section through the middle portion of the eye. The change in facet size is evident. (c) Section through the ventral portion of the eye. 
a)

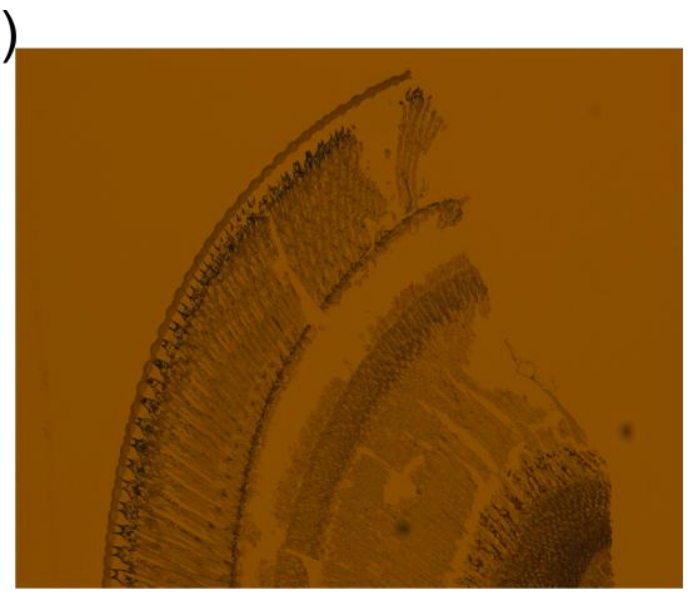

b)

c)

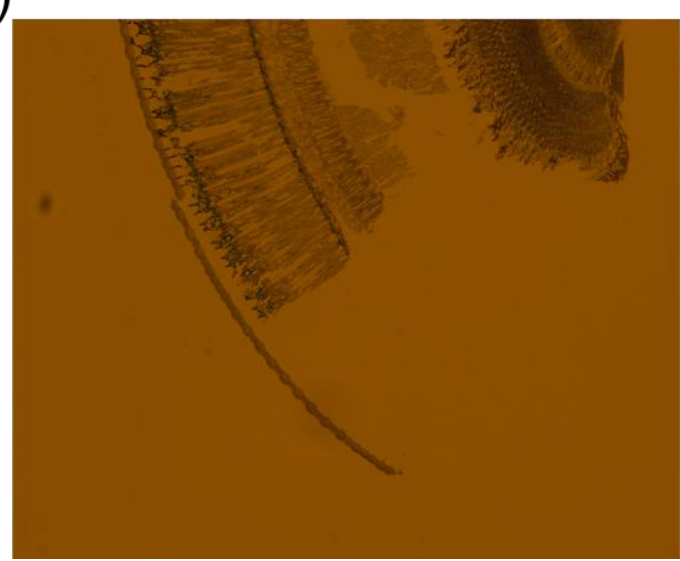

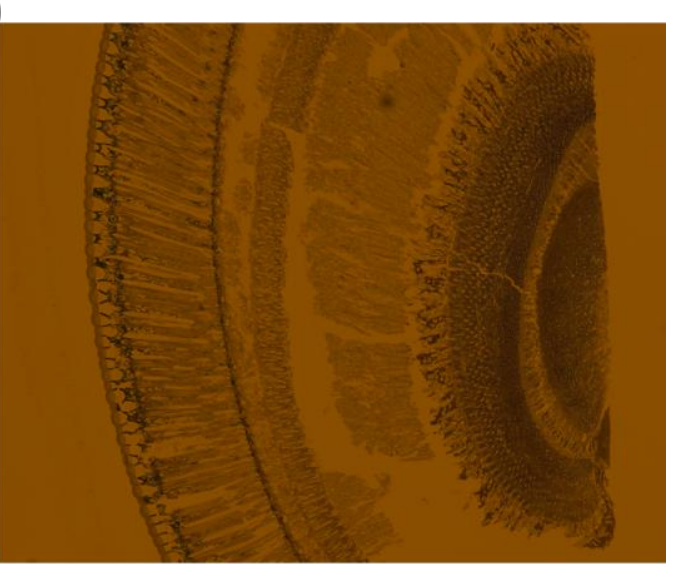

Fig. A.3. Female compound eye histology photos (100x). Sections are through the (a) dorsal, (b) middle, and (c) ventral portion of the eye. 
a)

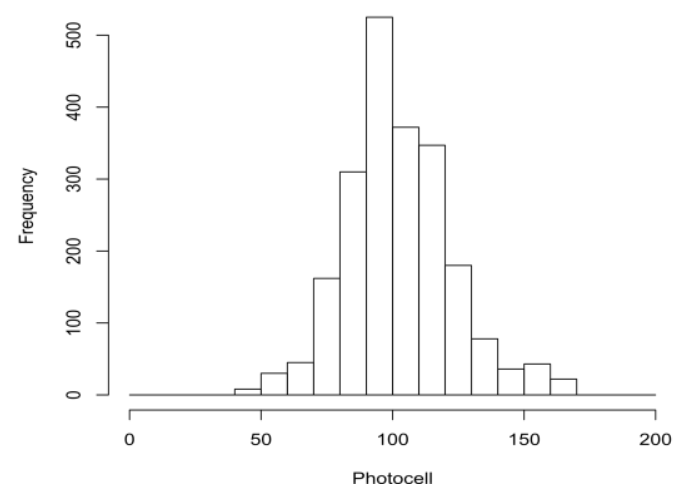

b)

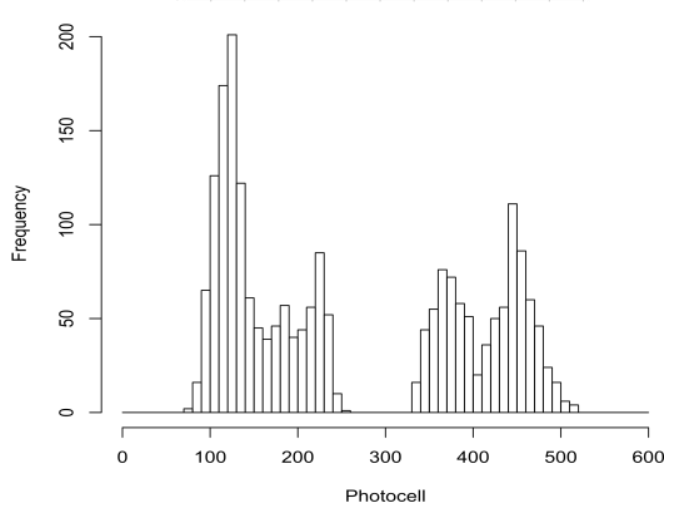

Fig. A.4. Histograms of the "photocell" readings when a pen or a tethered dead fly was passed through either the (a) flying or (b) walking sensor. 
a)

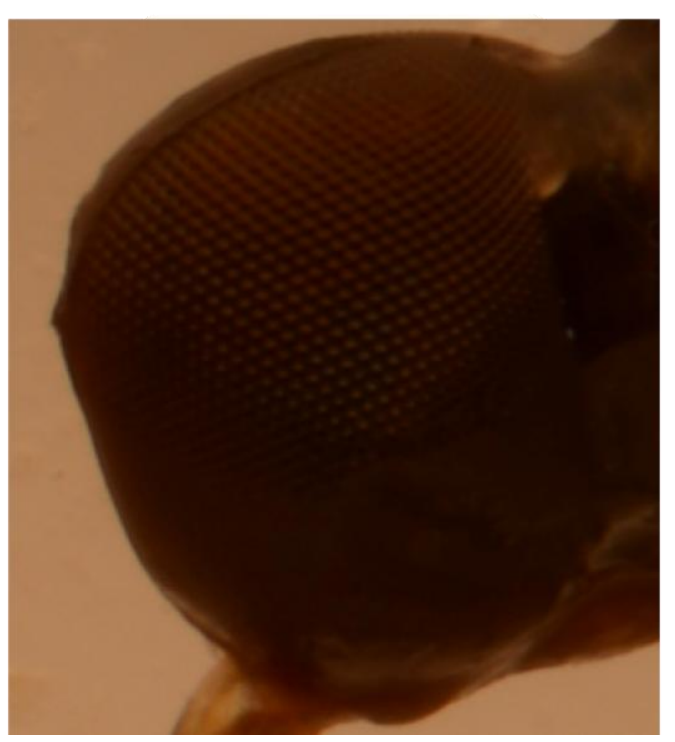

b)

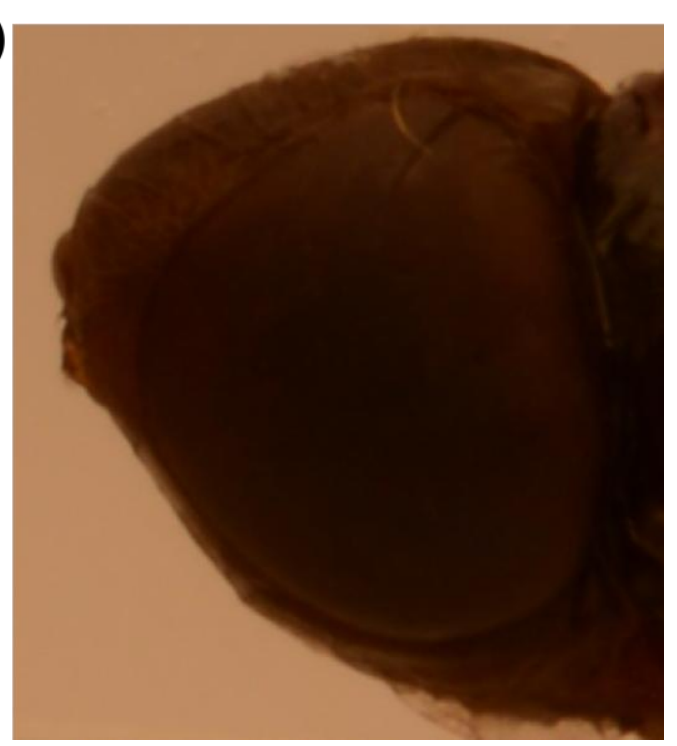

Fig. A.5. Comparison of (a) male and (b) female Chrysomya megacephala pharate adult compound eyes showing sexual dimorphism. The enlarged dorsal facets are visible in (a) allowing for sex to be determined. 
$\stackrel{20}{1}$

40

60

$\stackrel{80}{1}$

C_mega_tra_male_specific GTAATTTTCTTTACAAAATTAAAAAAGAAAGCACTTTGTTTTTTAGCTATTTCCAGTTTAGTGTTTTTTAATGATTATGAAAAAATTAATTTTACTTACG CATTAAAAGAATGTTTAATTTTTCTTTCGTGAAACAAAAAATCGATAAAGGTCAAATCACAAAAAATTACTAATACTTTTTAATTAAAATGAATGC

AT T T TGT TGGGAT T TGAAAT TAAAT T TAAAGAAAAAATATTAAAAT TATAAAATATGAAAAT T T TATAATGGTGT TAATGGCAACTGTAAAAGCAATGCT

C_mega_tra_male_specific ATTTTGTTGGGATTTGAA TAAAACAACCCTAAACT T TAATT TAAAT T TC TT T TT TATAAT T T TAATAT T T TATACT T T TAAAAT AT TACCACAAT TACCGTTGACAT T T TCGT TACGA

220

260

$\stackrel{280}{1}$

C_mega_tra_male_specific GGAAAAATGCTTATGCCGTAAGGCTTCTACAATCAACATACTAGATCTGGGCTAAAGGAAGGATCATATGGACATTCTATGGTAGTGGAATCAATGGATA CCTTTTTACGAATACGGCAT TCCGAAGATGTTAGTTGTATGATCTAGACCCGATTTCCTTCCTAGTATACCTGTAAGATACCATCACCTTAGTTACCTAT

C_mega_tra_male_specific TTACA

AATGT

Fig. A.6. The DNA sequence for the male specific exon in C. megacephala. The base pair corresponding to position one represents the portion of the sequence that is present just after exon 1 . The base pair corresponding to position 305 represents the portion of the sequence that is present just before exon 2 . 
Table A.1. Summary of gene expression as measured by band intensities for all 40 intrapuparial samples. The values are the average of two amplifications of ribosomal protein 49 (rp49) and rhodopsin 1 (rh1), with the band intensity for each sample divided by the ladder as described in the methods for Chapter V. The normalized $r h 1$ value is the average of the $r h 1$ value divided by the average of the rp49 value for that same sample.

\begin{tabular}{|c|c|c|c|c|c|}
\hline Sample & Time (hrs) & Sex & RP49 Average & Rh1 Average & Rh1 Normalized Expression \\
\hline 67 & 192 & male & 1.233355791 & 0.044728977 & 0.036014434 \\
\hline 68 & 192 & female & 1.31822894 & 0.047813616 & 0.035041767 \\
\hline 69 & 192 & male & 1.419447832 & 0.006032611 & 0.004101928 \\
\hline 70 & 192 & female & 1.128173154 & 0.040221134 & 0.042008902 \\
\hline 71 & 192 & female & 1.244914746 & 0.025229255 & 0.018942896 \\
\hline 72 & 192 & male & 1.684568109 & 0.112978562 & 0.068080471 \\
\hline 73 & 192 & male & 1.150973106 & 0.089375149 & 0.07871616 \\
\hline 74 & 192 & male & 1.508592573 & 0.001790943 & 0.001169726 \\
\hline 75 & 204 & female & 1.283280959 & 0.014872913 & 0.011463443 \\
\hline 76 & 204 & female & 1.2705212 & 0.055997917 & 0.04344612 \\
\hline 77 & 204 & female & 1.487277235 & 0.016678831 & 0.012338166 \\
\hline 78 & 204 & male & 1.298452779 & 0.682369854 & 0.526321021 \\
\hline 79 & 204 & male & 1.498966559 & 0.169071135 & 0.112397136 \\
\hline 80 & 204 & female & 1.241798369 & 0.005483136 & 0.004453145 \\
\hline 81 & 204 & male & 1.680644694 & 0.028616809 & 0.016278841 \\
\hline 82 & 204 & female & 1.455107434 & 0.00509157 & 0.00339641 \\
\hline 83 & 216 & male & 0.983243533 & 1.006763281 & 1.083809412 \\
\hline 84 & 216 & male & 1.375395102 & 0.904480358 & 0.656867754 \\
\hline 85 & 216 & female & 1.258981909 & 0.056170898 & 0.040090643 \\
\hline 86 & 216 & male & 1.510782767 & 0.093844087 & 0.063668461 \\
\hline 87 & 216 & female & 1.357787467 & 0.014162719 & 0.011310243 \\
\hline 88 & 216 & male & 1.496959652 & 0.129680328 & 0.087963448 \\
\hline 89 & 216 & male & 1.462630783 & 0.099849016 & 0.064719502 \\
\hline 90 & 216 & male & 1.347276389 & 0.053991434 & 0.038237114 \\
\hline 91 & 228 & male & 1.230283251 & 1.47555877 & 1.199315014 \\
\hline 92 & 228 & female & 1.170229957 & 0.98224094 & 0.838636176 \\
\hline 93 & 228 & female & 1.381508587 & 0.001850264 & 0.001358168 \\
\hline 94 & 228 & female & 1.102607601 & 0.933809047 & 0.846825633 \\
\hline 95 & 228 & male & 1.350608937 & 0.2208698 & 0.166285008 \\
\hline 96 & 228 & female & 1.350214281 & 0.607562608 & 0.447979122 \\
\hline 97 & 228 & female & 1.501208847 & 1.802713471 & 1.206392387 \\
\hline 98 & 228 & male & 1.40814971 & 0.351690672 & 0.240671059 \\
\hline 99 & 240 & female & 0.952994293 & 1.938905456 & 2.04814918 \\
\hline 100 & 240 & male & 1.353283403 & 0.656518436 & 0.485304578 \\
\hline 101 & 240 & male & 1.412542914 & 2.002454475 & 1.417969994 \\
\hline 102 & 240 & male & 1.32184315 & 2.36336377 & 1.788471931 \\
\hline 103 & 240 & male & 1.256922265 & 2.454470548 & 1.947750085 \\
\hline 104 & 240 & female & 1.506236092 & 0.045772597 & 0.030501379 \\
\hline 105 & 240 & male & 1.313295965 & 2.832251214 & 2.166757035 \\
\hline 106 & 240 & female & 1.160416051 & 1.279872016 & 1.129886145 \\
\hline
\end{tabular}


VITA

\section{JOSHUA L. SMITH}

Born, Lutz, Florida

2003-2007

B.S., Biology

Stetson University

DeLand, Florida

2007-2009

M.F.S., Forensic Sciences

George Washington University

Washington, D.C.

$2010-2011$

Doctoral Student, Biology

West Virginia University

Morgantown, West Virginia

2011-2016

Doctoral Student/Candidate, Biological Sciences

Florida International University

Miami, Florida

\section{PUBLICATIONS}

Smith, J.L., and Wells, J.D. (In press). Isolation of the male-specific transformer exon as method for immature specimen sex identification in Chrysomya megacephala (Diptera: Calliphoridae). Journal of Medical Entomology doi:10.1093/jme/tjw198.

Smith, J.L., Palermo, N.A., Theobald, J.C., and Wells, J.D. (2016). The forensically important blow fly, Chrysomya megacephala (Diptera: Calliphoridae), is more likely to walk than fly to carrion at low light levels. Forensic Science International 266: 245-249.

Smith, J.L., Palermo, N.A., Theobald, J.C., and Wells, J.D. (2015). Body size, rather than male eye allometry, explains Chrysomya megacephala (Diptera: Calliphoridae) activity in low light. Journal of Insect Science 15(1): 133. DOI: 10.1093/jisesa/iev114.

Wells, J.D., and Smith, J.L. (2013). First case report of Blaesoxipha plinthopyga (Diptera: Sarcophagidae) from a human corpse in the USA and new state record based on specimen genotype. Journal of Forensic Sciences 58(5): 1378-1380. 